TOKYO J. MATH.

Vol. 7, No. 1, 1984

\title{
Classification of Periodic Maps on Compact Surfaces: II
}

\author{
Kazuo YOKOYAMA \\ Sophia Univerrsity
}

\section{Introduction}

In [7], we have classification of orientation preserving periodic maps on compact orientable surfaces. In this paper, we will obtain classification of orientation reversing periodic maps on compact orientable surfaces and classification of periodic maps on compact non-orientable surfaces. We use the definitions and notations in [7]. We will assume that all surfaces are connected.

Let $P_{n}^{-}$be the set of elements $(f, M) \in P_{n}$ where $M$ is an orientable surface and $f$ is an orientation reversing periodic map. Let $P_{n}^{0}$ be the set of elements $(f, M) \in P_{n}$ where $M$ is a non-orientable surface. Then we will obtain classifications of $P_{n}^{-}$and $P_{n}^{0}$. So, we will complete the classification of the set $P_{n}$ of elements $(f, M)$ such that $\mathscr{S}(f)$ consists of finite points in $\stackrel{\circ}{M}$ (may be empty). Complete classification of periodic maps on compact surfaces will be given in the forthcoming paper [8].

For an element $(f, M)$ of $P_{n}$, we will consider its orbit space $X=$ $M / f$ and the canonical map $p: M \rightarrow X$. Then, by [4], $X$ is a compact surface and $p$ is an $n$-fold cyclic branched cover of $X$ with branched set $S=p(\mathscr{S}(f))$. We denote by $P_{n}(X, S)$ the set of elements $(f, M)$ of $P_{n}$ such that $X=M / f$ and $p: M \rightarrow X$ is an $n$-fold cyclic branched cover of $X$ with branched set $S$. For the classification of $P_{n}$, we will determine a complete set of the equivalence classes of $P_{n}(X, S)$ in $\S \S 2,3$ and 4 (see Theorems 2.1, 2.2, 3.1, 3.2. 4.1 and 4.2), which is of importance in the sequel.

Let $P_{n}^{e}(\widetilde{g}, \tilde{l}, \tilde{m}, \tilde{z}, \tilde{m})$ be the set of elements $(f, M)$ of $P_{n}^{\varepsilon}$, (where $\varepsilon=$ - or 0), satisfying the following conditions;

(1) $M$ is a compact surface of genus $\widetilde{g}$ with the boundary components $D_{1}, D_{2}, \cdots, D_{i}$,

(2) $f$ is a periodic map on $M$ such that $\mathscr{S}(f)$ consists $\tilde{m}$ points $S_{1}, S_{2}, \cdots, S_{\tilde{m}}$ in $\stackrel{\circ}{M}$,

(3) $\tilde{l}=\left(\tilde{l}_{a}\right)_{a \mid n}$ is a vector of non-negative integers $\tilde{l}_{a}$, where $\tilde{l}_{a}$ is 
the number of elements of the set $\left\{D_{j} ; f^{a}\left(D_{j}\right)=D_{j}\right.$ and $f^{b}\left(D_{j}\right) \neq D_{j}$ for $1 \leqq b<a\}$ for each divisor $a$ of $n$,

(4) $\tilde{m}=\left(\tilde{m}_{a}\right)_{\substack{a \mid n \\ a \neq n}}$ is a vector of non-negative integers $\tilde{m}_{a}$, where $\widetilde{m}_{a}$ is the number of elements of the set $\mathscr{S}_{a}(f)=\left\{S_{k} ; f^{a}\left(S_{k}\right)=S_{k}\right.$ and $f^{b}\left(S_{k}\right) \neq S_{k}$ for $1 \leqq b<a\}$ for eace divisor $a$ of $n$ except $n$. Denote by $\mathscr{P}_{n}^{\varepsilon}(\widetilde{g}, \widetilde{l}, \tilde{m}, \tilde{z}, \tilde{m})$ the set of equivalence classes of $P_{n}^{c}(\widetilde{g}, \widetilde{l}, \tilde{m}, \tilde{l}, \tilde{m})$.

Using the orbit space $M / f$ and the branched cover $p: M \rightarrow M / f$, we will obtain the following;

Proposition 5.1. If $P_{n}^{0}(\widetilde{g}, \tilde{l}, \tilde{m}, \tilde{l}, \tilde{m}) \neq \varnothing$, then we have

(1) $\tilde{l}=\sum_{a \mid n} \widetilde{l}_{a}$ and $\tilde{m}=\sum_{\substack{a \mid n \\ a \neq n}} \tilde{m}_{a}$,

(2) $\tilde{l}_{a} \equiv 0(\bmod a)$ for each divisor $a$ of $n$ and $\tilde{m}_{a} \equiv 0(\bmod a)$ for each divisor a of $n$ except $n$,

(3) $\widetilde{g}-2+\sum_{a \mid n}(1-n / a)\left(\widetilde{l}_{a}+\tilde{m}_{a}\right)+2 n$ is a positive integer and a multiple of $n$.

Then, let $l_{a}=\tilde{l}_{a} / a, m_{a}=\tilde{m}_{a} / a$, and $g=(1 / n)\left\{\tilde{g}-2+\sum_{a \mid n}(1-n / a)\left(\tilde{l}_{a}+\tilde{m}_{a}\right)+\right.$ $2 n\}$. We will prove the following;

Theorem A.1. Under the conditions (1), (2) and (3) in Proposition 5.1 , in order that $P_{n}^{0}(\widetilde{g}, \widetilde{l}, \tilde{m}, \tilde{\ell}, \tilde{m})$ be non-empty, it is necessary and sufficient that $n, \varangle$ and $m$ satisfy the following conditions;

(a) In case that $g \geqq 3$,

( I ) $n$ is odd, or

(II) $\sum_{\substack{a ! n \\ a \text {.ndd }}}\left(l_{a}+m_{a}\right)$ is even, if $n$ is even,

(b) In case that $g=1$,

(I ) g.c.d. $\left\{a ; l_{a} \neq 0\right.$ or $\left.m_{a} \neq 0\right\}=1$, if $n$ is odd, or

(II) $\sum_{\substack{a \mid n \\ a: \text { odd }}}\left(l_{a}+m_{a}\right)$ is even and g.c.d. $\left\{a ; l_{a} \neq 0\right.$ or $\left.m_{a} \neq 0\right\}=1$, if $n$ is even,

(c) In case that $g=2$,

(I ) $n$ is odd,

(II) $\sum_{a \mid n}\left(l_{a}+m_{a}\right)$ is even, if $n$ is even and $d$ is odd,

(III) $n / 2$ is odd, if $n$ is even and $d$ is even, or

(IV) $d / 2$ is odd and $\sum_{\substack{a|n \\ a| \text { in } \\ a \mid 2 \text { : odd }}}\left(l_{a}+m_{a}\right)$ is odd, if $n$ is even, $d$ is even and $n / 2$ is even,

where $d=$ g.c.d. $\left\{a ; l_{a} \neq 0\right.$ or $\left.m_{a} \neq 0\right\}$.

TheOREM A.2. Suppose that $n, \tilde{g}, \tilde{l}, \tilde{m}, \tilde{z}$ and $\tilde{m}$ satisfy the conditions in Theorem A.1. Then the number of elements of $\mathscr{P}_{n}^{0}(\widetilde{g}, \widetilde{l}, \tilde{m}, \tilde{z}, \tilde{m})$ is given as follows; 
(a) In case that $g \neq 2$,

$\left\{C(n ; \chi, m)\right.$, if (I) $n$ is odd or (II) $n$ is even and $l_{n / 2}+m_{n / 2} \neq 0$; $\left\{\times C(n ; \chi, m)\right.$, if $n$ is even and $l_{n / 2}=m_{n / 2}=0$.

(b) In case that $g=2$, $\{\varphi(d) / 2\} \times C(n ; \chi, m)$, if $n$ is odd;

$2 \times\{\varphi(d) / 2\} \times C(n ; \gamma, m)$, if $n$ is even, $d$ is odd and $l_{n / 2}=m_{n / 2}=0$;

$\{\varphi(d) / 2\} \times C(n ; \chi, m)$, if $n$ is even, $d$ is odd and $l_{n / 2}+m_{n / 2} \neq 0$;

$\{\varphi(d / 2) / 2\} \times C(n ; \gamma, m)$, if $n$ is even, $d$ is even and $n / 2$ is odd;

$\{2 \times\{\varphi(d / 2) / 2\} \times C(n ; \downarrow, m)$, if $n$ is even, $d$ is even, $n / 2$ is even and $l_{n / 2}=m_{n / 2}=0$;

$\{\varphi(d / 2) / 2\} \times C(n ; \downarrow, m)$, if $n$ is even, $d$ is even, $n / 2$ is even and $l_{n / 2}+m_{n / 2} \neq 0$

where $\{x\}$ is the smallest integer $\geqq x, \varphi(x)$ is the Euler function and

$$
C(n ; \varnothing, m)=\prod_{\substack{a \mid n \\
a \neq n \\
a \neq n: 2}}\left(\begin{array}{c}
\frac{\varphi(n / a)}{2}+l_{a}-1 \\
l_{a}
\end{array}\right)\left(\begin{array}{c}
\frac{\varphi(n / a)}{2}+m_{a}-1 \\
m_{a}
\end{array}\right) .
$$

In the case of $P_{n}^{-}(\widetilde{g}, \tilde{l}, \tilde{m}, \tilde{l}, \tilde{m})$, we will obtain the following;

Proposition 6.1. If $P_{n}^{-}(\tilde{g}, \tilde{l}, \tilde{m}, \tilde{\ell}, \tilde{m}) \neq \varnothing$, then we have

(0) $n$ is even,

(1) $\quad \widetilde{l}=\sum_{a \mid n} \tilde{l}_{a}$ and $\tilde{m}=\sum_{\substack{a \mid n \\ a \neq n}} \tilde{m}_{a}$,

(2) $\tilde{l}_{a} \equiv 0(\bmod a)$ for each divisor $a$ of $n$ and $\tilde{m}_{a} \equiv 0(\bmod a)$ for each divisor $a$ of $n$ except $n$,

$(3)^{\prime} 2 \widetilde{g}-2+\sum_{a \mid n}(1-n / a)\left(\widetilde{l}_{a}+\widetilde{m}_{a}\right)+2 n$ is a positive integer and a multiple of $2 n$,

(4) $\widetilde{l}_{a}=\widetilde{m}_{a}=0$ for each odd divisor $a$ of $n$.

Then, let $l_{a}=\tilde{l}_{a} / a, m_{a}=\tilde{m}_{a} / a$ and $g=(1 / 2 n)\left\{2 \widetilde{g}-2+\sum_{a \mid n}(1-n / a)\left(\tilde{l}_{a}+\right.\right.$ $\left.\left.\tilde{m}_{a}\right)+2 n\right\}$. We will prove the following;

THEOREM B.1. Under the conditions (0), (1), (2), (3)' and (4) in Proposition 6.1, in order that $P_{n}^{-}(\widetilde{g}, \tilde{l}, \tilde{m}, \tilde{\&}, \tilde{m})$ be non-empty, it is necessary and sufficient that $n, \&$ and $m$ satisfy the following conditions;

(a) In case that $g$ is odd and $g \geqq 3$,

(I) $n / 2$ is odd, or

(II) $\sum_{\substack{a \mid n \\ a: \text { even } \\ a: 2: \text { odd }}}\left(l_{a}+m_{a}\right)$ is odd, if $n / 2$ is even,

(b) In case that $g=1$,

(I) $(1 / 2) \times$ g.c.d. $\left\{a ; l_{a} \neq 0\right.$ or $\left.m_{a} \neq 0\right\}=1$, if $n / 2$ is odd, or 


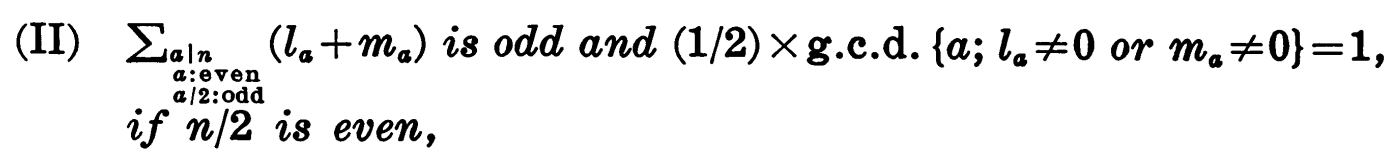

(c) In case that $g$ is even,

(I) $n / 2$ is odd, or

(II) $\sum_{\substack{a \mid n \\ a: \text { even } \\ a \mid 2: \text { odd }}}\left(l_{a}+m_{a}\right)$ is even, if $n / 2$ is even.

THEOREM B.2. Suppose that $n, \tilde{g}, \tilde{l}, \tilde{m}, \tilde{z}$ and $\tilde{m}$ satisfy the conditions in Theorem B.1. Then, the number of elements of $\mathscr{P}_{n}^{-}(\widetilde{g}, \tilde{l}, \tilde{m}$, $\tilde{\mathrm{z}}, \tilde{\mathrm{m}})$ is given as follows;

(a) In case that $g \neq 2$,

$\left\{C(n ; \downarrow, m)\right.$, if (I) $n / 2$ is odd or (II) $n / 2$ is even and $l_{n / 2}+m_{n / 2} \neq 0$;

$\left\{2 \times C(n ; \gamma, m)\right.$, if $n / 2$ is even and $l_{n / 2}=m_{n / 2}=0$.

(b) In case that $g=2$,

$\{\varphi(d) / 2\} \times C(n ; l, m)$, if $n / 2$ is odd;

$\{(\varphi(d)+\varphi(d / 2)) / 2\} \times C(n ; \tilde{z}, m)$, if $n / 2$ is even and $n / d$ is odd;

$2 \times\{\varphi(d) / 2\} \times C(n ; \chi, m)$, if $n / 2$ is even, $d / 2$ is odd and $l_{n / 2}=m_{n / 2}=0$;

$\{\varphi(d) / 2\} \times C(n ; l, m)$, if $n / 2$ is even, $d / 2$ is odd and $l_{n / 2}+_{n / 2} \neq 0$;

$2 \times\{\varphi(d) / 2\} \times C(n ; \gamma, m)$, if $n / 2$ is even, $d / 2$ is even; $n / d$ is even, $\sum_{a \mid n=0 \text { odd }}\left(l_{a}+m_{a}\right)$ is even and $l_{n / 2}=m_{n / 2}=0$;

$\{\{(d) / 2\} \times C(n ; \gamma, m)$, if $n / 2$ is even, $d / 2$ is even, $n / d$ is even, $\sum_{\substack{a ! n \\ a \cdot d: \text { odd }}}\left(l_{a}+m_{a}\right)$ is even and $l_{n / 2}+m_{n / 2} \neq 0$;

$2 \times\{\varphi(d / 2) / 2\} \times C(n ; \chi, m)$, if $n / 2$ is even, $d / 2$ is even, $n / d$ is even, $\sum_{a \mid n}\left(l_{a}+m_{a}\right)$ is odd and $l_{n / 2}=m_{n / 2}=0$;

$\{\varphi(d / 2) / 2\} \times C(n ; \ell, m)$, if $n / 2$ is even, $d / 2$ is even, $n / d$ is even, $\sum_{\substack{a|n \\ a| d: \text { odd }}}\left(l_{a}+m_{a}\right)$ is odd and $l_{n / 2}+m_{n / 2} \neq 0$;

where $\{x\}, \varphi(x)$ and $C(n ; \gamma, m)$ is the same notations in Theorem A.2, and $d=$ g.c.d. $\left\{a ; l_{a} \neq 0\right.$ or $\left.m_{a} \neq 0\right\}$. [1].

In the case of $n=2$ and $m=0$, Theorems $\mathrm{A}$ and $\mathrm{B}$ is given by Asoh

In $\S 1$, we will give a model of $(X, S)$ and reduce the equivalence relation on $P_{n}(X, S)$ in a similar way as in [7]. In $\S \S 2,3$ and 4 , we will determine the equivalence classes of $P_{n}(X, S)$ using the homeotopy group of $(X, S)$, and prove Theorems $2.1,2.2,3.1,3.2,4.1$ and 4.2. In $\S 5$ we will prove Theorems A.1 and A.2 and in $\S 6$ we will prove Theorems B.1 and B.2. Not only we can determine the number of elements of $\mathscr{P}_{n}^{0}(\widetilde{g}, l, \tilde{m}, \tilde{\mathbb{z}}, \tilde{m})$ or $\mathscr{P}_{n}^{-}(\widetilde{g}, \tilde{l}, \tilde{m}, \tilde{z}, \tilde{m})$, but also construct an element of $\mathscr{P}_{n}^{0}(\widetilde{g}, \tilde{l}, \tilde{m}, \tilde{l}, \tilde{m})$ or $\mathscr{P}_{n}^{-}(\widetilde{g}, \tilde{l}, \tilde{m}, \tilde{l}, \tilde{m})$ in practice. Moreover, we can 
determine whether two elements of $P_{n}^{0}$ (resp. $P_{n}^{-}$) are equivalent or not.

The author would like to express his sincere gratitude to Prof. Tatsuo Homma and Prof. Shin'ichi Suzuki for helpful conversations.

§ 1. A model for $X$ and reductions of equivalence relation on $P_{n}(X, S)$.

Let $X_{2 g+1}$ (resp. $X_{2 g+2}$ ) be a compact connected non-orientable surface of genus $2 g+1$ (resp. $2 g+2$ ) and let the boundary $\partial X_{2 g+1}$ (resp. $\partial X_{2 g+2}$ ) consist of $l$ components $\widetilde{d}_{1}, \widetilde{d}_{2}, \cdots, \widetilde{d}_{l}$. For the sake of convenience, we first take a model for $X_{2 g+1}$ (resp. $X_{2 g+2}$ ) as shown in Fig. 1 (resp. Fig. 2), and simple oriented loops $a_{1}, a_{2}, \cdots, a_{g}, b_{1}, b_{2}, \cdots, b_{g}, c$, (resp. $c_{1}, c_{2}$ ), $d_{1}$, $d_{2}, \cdots, d_{l}$ on $X_{2 g+1}$ (resp. $X_{2 g+2}$ ). Let $S$ be finite points $\widetilde{s}_{1}, \widetilde{s}_{2}, \cdots, \widetilde{s}_{m}$ in ${\stackrel{\circ}{X_{2 g+1}}}$ (resp. $\stackrel{\circ}{X}_{2 g+2}$ ) and take simple oriented loops $s_{1}, s_{2}, \cdots, s_{m}$ on $X_{2 g+1}$ (resp. $X_{2 g+2}$ ) as shown in Fig. 1 (resp. Fig. 2).

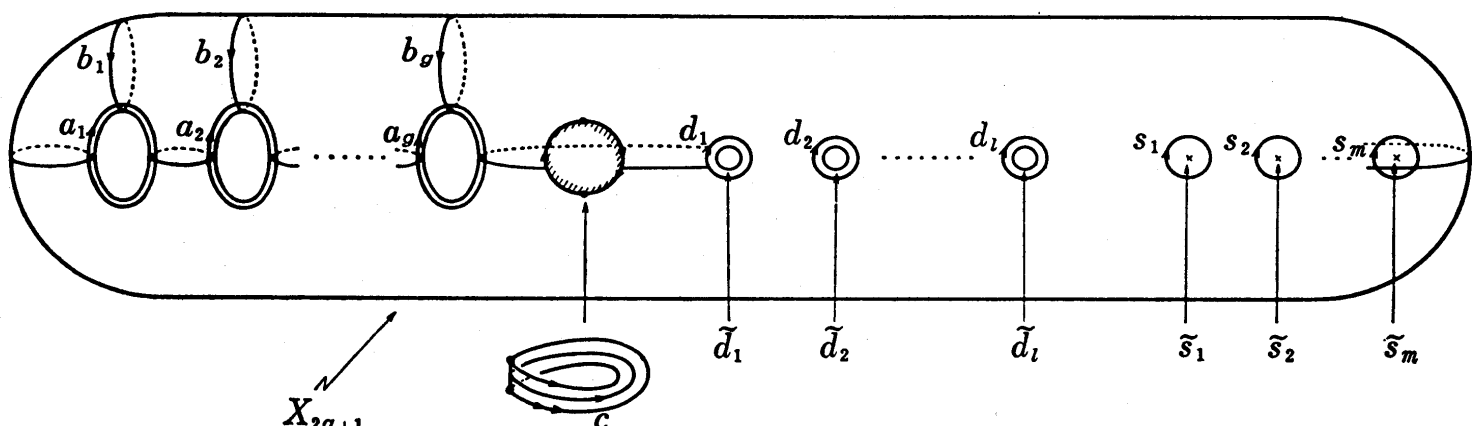

FiguRe 1

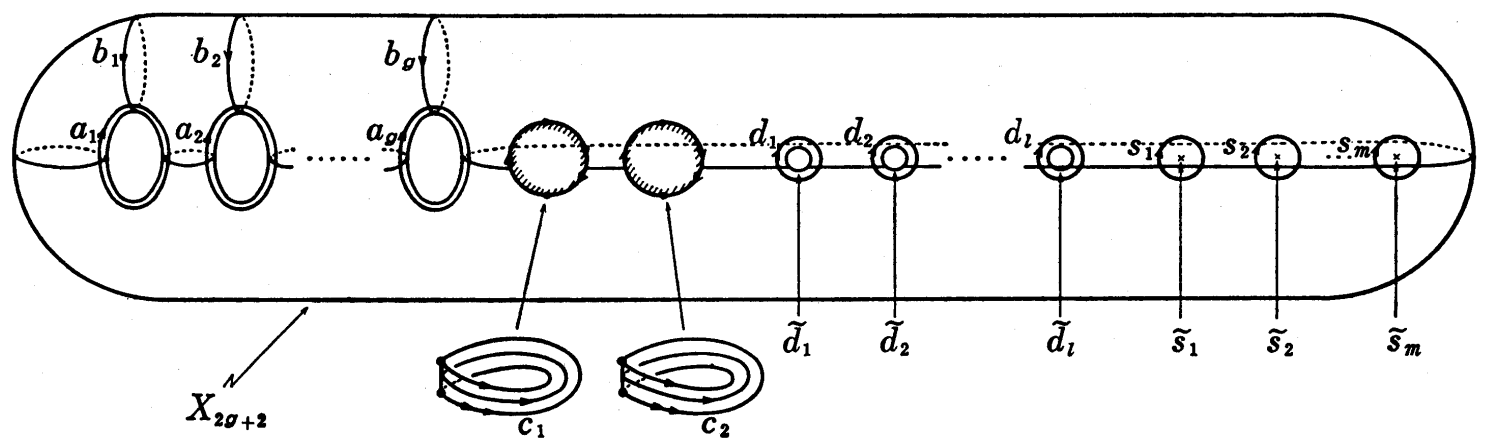

Figure 2

To avoid the multiplicity of brackets, we refer to loops rather than to homology classes. Then the first integral homology group of $X_{2 g+1}-S$ is given by; 


$$
H_{1}\left(X_{2 g+1}-S\right)=\left|\begin{array}{lr}
a_{1}, b_{1}, a_{2}, b_{2}, \cdots, a_{g}, b_{g}, & 2 c+d_{1}+d_{2}+\cdots+d_{l} \\
c, & +s_{1}+s_{2}+\cdots+s_{m}=0 \\
d_{1}, d_{2}, \cdots, d_{l}, & ; \\
s_{1}, s_{2}, \cdots, s_{m} &
\end{array}\right| .
$$

The first integral homology group of $X_{2 g+2}-S$ is given by;

$$
H_{1}\left(X_{2 g+2}-S\right)=\left(\begin{array}{lc}
a_{1}, b_{1}, a_{2}, b_{2}, \cdots, a_{g}, b_{g}, & \\
c_{1}, c_{2}, & 2 c_{1}+2 c_{2}+d_{1}+d_{2}+\cdots+d_{l} \\
d_{1}, d_{2}, \cdots, d_{l}, & +s_{1}+s_{2}+\cdots+s_{m}=0 \\
s_{1}, s_{2}, \cdots, s_{m}
\end{array} \mid .\right.
$$

By the same way as in [7], we define $\left[H_{1}(X-S) ; Z_{n}\right]^{*}$ and $\mathscr{A}$-equivalence relation on $\left[H_{1}(X-S) ; Z_{n}\right]^{*}$ (see Definition 1 in [7]), where $X=X_{2 g+1}$ or $X_{2 g+2}$. To avoid a multiplicity of $*$ we also use $h$ as a homomorphism $h_{*}$ induced by a homeomorphism $\left.h\right|_{x_{-S}}$, if there is no confusion. We have the same result as Proposition 2 in [7].

Proposition 1.1. There is a one-to-one correspondence between the set of equivalence classes of $P_{n}(X, S)$ and the set of $\mathscr{A}$-equivalence classes of $\left[H_{1}(X-S) ; Z_{n}\right]^{*}$.

Let $Z_{n}^{-}(2 g+1 ; l, m)$ be a set of systems of integers $(\alpha, \beta, \gamma, \delta, \theta)=$ $\left(\alpha_{1}, \beta_{1}, \alpha_{2}, \beta_{2}, \cdots, \alpha_{g}, \beta_{g}, \gamma, \delta_{1}, \delta_{2}, \cdots, \delta_{l}, \theta_{1}, \theta_{2}, \cdots, \theta_{m}\right)$ satifying the following conditions; $1,2, \cdots, m)$,

(0) $\alpha_{i}, \beta_{i}, \gamma, \delta_{j}, \theta_{k} \in Z_{n}$ and $\theta_{k} \neq 0 \quad(i=1,2, \cdots, g ; j=1,2, \cdots, l ; k=$

(1) $2 \gamma+\delta_{1}+\delta_{2}+\cdots+\delta_{l}+\theta_{1}+\theta_{2}+\cdots+\theta_{k} \equiv 0(\bmod n)$,

(2) g.c.d. $\left\{\alpha_{1}, \beta_{1}, \alpha_{2}, \beta_{2}, \cdots, \alpha_{g}, \beta_{g}, \gamma, \delta_{1}, \delta_{2}, \cdots \delta_{l}, \theta_{1}, \theta_{2}, \cdots, \theta_{m}, n\right\}=1$, where g.c.d. means the greatest common divisor.

Let $Z_{n}^{-}(2 g+2 ; l, m)$ be a set of systems of integers $\left(\alpha, \beta, \gamma_{1}, \gamma_{2}, \delta, \theta\right)=$ $\left(\alpha_{1}, \beta_{1}, \alpha_{2}, \beta_{2}, \cdots, \alpha_{g}, \beta_{g}, \gamma_{1}, \gamma_{2}, \delta_{1}, \delta_{2}, \cdots, \delta_{l}, \theta_{1}, \theta_{2}, \cdots, \theta_{m}\right)$ satisfying the following conditions;

( 0 ) $\alpha_{i}, \beta_{i}, \gamma_{1}, \gamma_{2}, \delta_{j}, \theta_{k} \in Z_{n}$ and $\theta_{k} \neq 0(i=1,2, \cdots, g ; j=1,2, \cdots, l ; k=$ $1,2, \cdots, m)$,

(1) $2 \gamma_{1}+2 \gamma_{2}+\delta_{1}+\delta_{2}+\cdots+\delta_{l}+\theta_{1}+\theta_{2}+\cdots+\theta_{k} \equiv 0(\bmod n)$, $n\}=1$.

(2) g.c.d. $\left\{\alpha_{1}, \beta_{1}, \alpha_{2}, \beta_{2}, \cdots, \alpha_{g}, \beta_{g}, \gamma_{1}, \gamma_{2}, \delta_{1}, \delta_{2}, \cdots, \delta_{l}, \theta_{1}, \theta_{2}, \cdots, \theta_{m}\right.$,

By the same way as in [7] (see pp. 78 and 79 in [7]), the map $\Sigma$ gives a one-to-one correspondence between $\left[H_{1}\left(X_{2 g+1}-S\right) ; Z_{n}\right]^{*}$ and $Z_{n}^{-}(2 g+$ $1 ; l, m)\left(\right.$ resp. $\left[H_{1}\left(X_{2 g+2}-S\right) ; Z_{n}\right]^{*}$ and $\left.Z_{n}^{-}(2 g+2 ; l, m)\right)$. 
The equivalence relation on $Z_{n}^{-}(2 g+1 ; l, m)\left(\operatorname{resp} . Z_{n}^{-}(2 g+2 ; l, m)\right)$ is defined by the same way as Definition 2 in [7]. Hence $\Sigma$ is a one-to-one correspondence between the set of $\mathscr{A}$-equivalence classes of $\left[H_{1}\left(X_{2 g+1}-S\right)\right.$; $\left.Z_{n}\right]^{*}$ (resp. $\left.\left[H_{1}\left(X_{2 g+2}-S\right) ; Z_{n}\right]^{*}\right)$ and the set of equivalence classes of $Z_{n}^{-}(2 g+1 ; l, m)\left(\right.$ resp. $\left.Z_{n}^{-}(2 g+2 ; l, m)\right)$.

§. Determination of the equivalence classes of $P_{n}\left(X_{2 g+1}, S\right)$.

In this section, we merely denote $X_{2 g+1}$ by $X$. To determine the equivalence classes of $P_{n}(X, S)$, we use the following result of Lickorish [3] and Chillingworth [2].

Proposition 2.1. There exists a $Y$-homeomorphism $\mathscr{Y}_{1}$ of $(X, S)$ onto itself such that the automorphism of $H_{1}(X-S)$ induced by it is given by;

$$
\mathscr{Y}_{1}\left(a_{1}\right)=a_{1}, \quad \mathscr{Y}_{1}\left(b_{1}\right)=-b_{1}+2 c, \quad \mathscr{Y}_{1}(c)=c,
$$

where the remaining generators of (1.1) are unchanged, see Fig. 3.

We use some typical homeomorphisms of surfaces in addition to [7].

Definition 2.1. Let $A, A_{1}, B_{+}, \psi$ be the same sets and map as Definition 3 in [7].
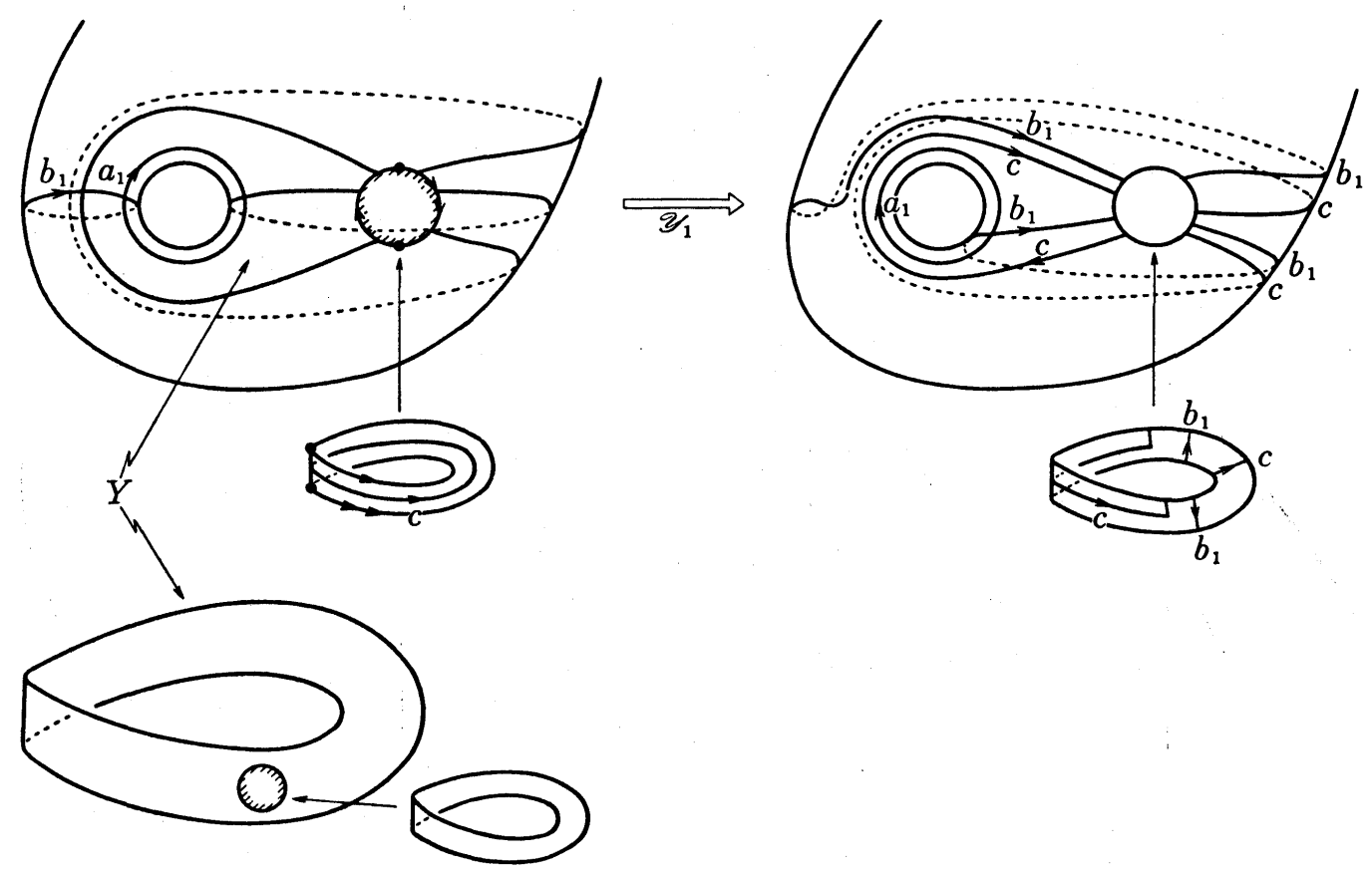

FIGURE 3 
(1) $\partial_{r}$ : We take a 2-cell $\Delta$ and identify $\partial \Delta$ with a component $\widetilde{d}_{1}$ of $\partial X$. We obtain the surface $X \cup \Delta$ of genus $2 g+1$ with $l-1$ boundary components. Let $e$ be a simple loop on $X \cup \Delta$ passing through the center of $\Delta$ such that $e \cap\left\{a_{1}, b_{1}, \cdots, a_{g}, b_{g}, c, d_{1}, d_{2}, \cdots d_{l}, s_{1}, s_{2}, \cdots, s_{m}\right\}=e \cap\left\{c, d_{1}\right\}$ and that $e$ intersects transversally one point with $c$; see Fig. 4. Let $h$ be an embedding of $A-A_{1}$ in $X \cup \Delta-S$ satisfying the following conditions; (1) $h(\widetilde{e})=e$, (2) $h\left(A-A_{1}\right)$ is a regular neighborhood of $e$, (3) $h(A-$ $\left.A_{1}\right) \cap\left\{a_{1}, b_{1}, \cdots, a_{g}, b_{g}, c, d_{1}, d_{2}, \cdots, d_{l}\right\}=h\left(A-A_{1}\right) \cap\left\{c, d_{1}\right\}$ and (4) $h\left(B_{+}\right)=\Delta$, where $\tilde{e}=\{(r, \theta) ; r=3\}$. Then we have a homeomorphism $\partial_{r}$ of $(X, S)$ onto itself defined as follows; $\partial_{r}=h \psi h^{-1}$ on $h\left(A-A_{1}\right)$ and $\partial_{r}$ is the identity on $X-h\left(A-A_{1}\right)$; see Fig. 4.

(2) $\sigma_{r}$ : We take a 2-cell $\Delta$ in $X$ such that $\stackrel{\supset}{\supset} s_{1}, \Delta \cap\left\{a_{1}, b_{1}, \cdots\right.$,
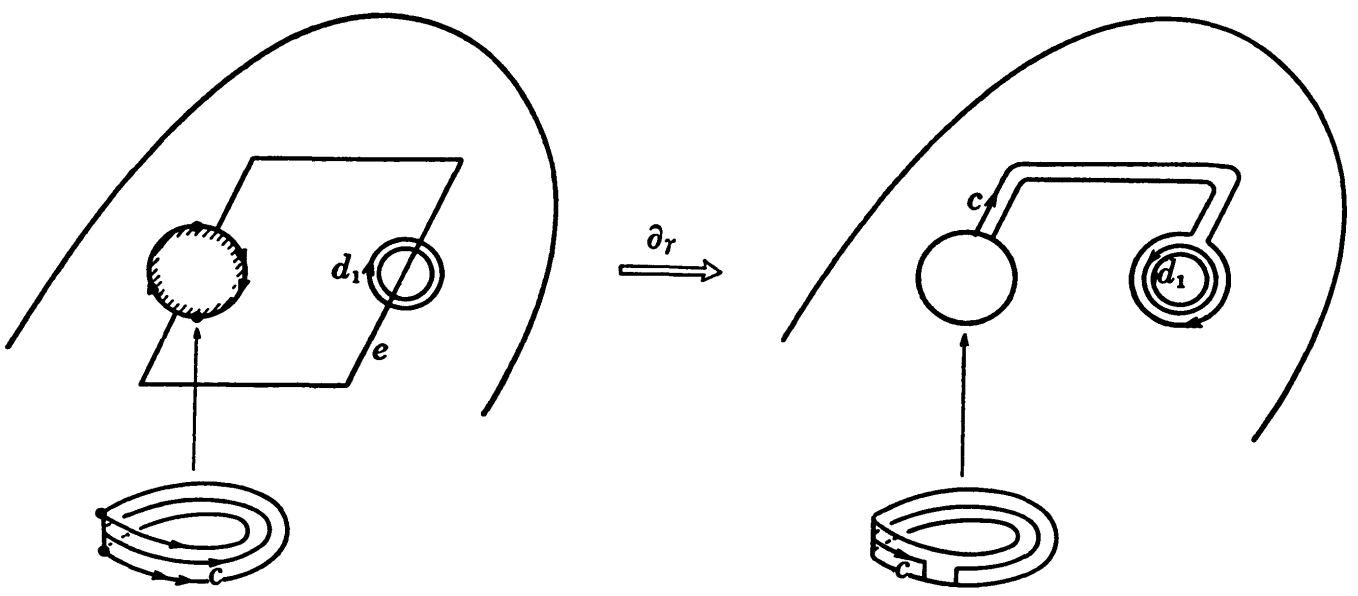

Figure 4
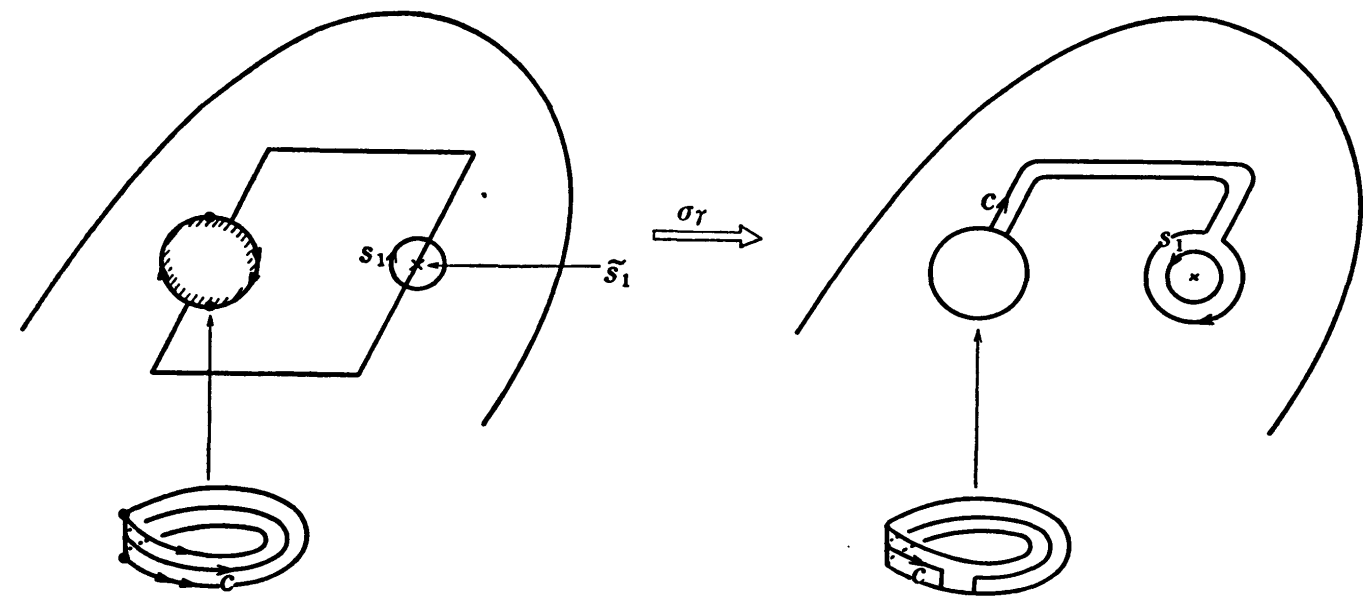

Figure 5 
$\left.a_{g}, b_{g}, c, s_{1}, s_{2}, \cdots, s_{m}\right\}=\left\{s_{1}\right\}$ and $\Delta \cap S=\left\{\widetilde{s}_{1}\right\}$. Let $e$ be a simple loop on $X$ passing through $\widetilde{s}_{1}$ such that $e \cap\left\{a_{1}, b_{1}, \cdots, a_{g}, b_{g}, c, d_{1}, d_{2}, \cdots, d_{l}, s_{1}, s_{2}, \cdots\right.$, $\left.s_{m}\right\}=e \cap\left\{c, s_{1}\right\}$ and that $e$ intersects transversally one point with $c$, see Fig. 5. Let $h$ be an embedding of $A-A_{1}$ in $X$ satisfying the following conditions; (1) $h(\widetilde{e})=e$, (2) $h\left(A-A_{1}\right)$ is a regular neighborhood of $e$, (3) $h\left(A-A_{1}\right) \cap\left\{a_{1}, b_{1}, \cdots, a_{g}, b_{g}, c, s_{1}, s_{2}, \cdots, s_{m}\right\}=h\left(A-A_{1}\right) \cap\left\{c, s_{1}\right\}$ and (4) $h\left(B_{+}\right)=\Delta$. Then we have a homeomorphism $\sigma_{r}$ of $(X, S)$ onto itself defined as follows; $\sigma_{\gamma}=h \psi h^{-1}$ on $h\left(A-A_{1}\right)$ and $\sigma_{\gamma}$ is the identity on $X-h\left(A-A_{1}\right)$; see Fig. 5.

Then, by Suzuki [5] and Chillingworth [2] and in an elementary way, we have the following;

Proposition 2.2. The homeotopy group of $(X, S)$ is generated by $\rho, \rho_{1 i}(2 \leqq i \leqq g), \tau_{1}, \mu_{1}, \theta_{12}, \mathscr{Y}_{1}, \partial_{j}(2 \leqq j \leqq l), \sigma_{k}(2 \leqq k \leqq m), \partial_{a}, \sigma_{a}, \partial_{r}$ and $\sigma_{\gamma}$.

LEMMA 2.1. (1) The automorphisms of $H_{1}(X-S)$ induced by them are given by;

$$
\begin{array}{ll}
\partial_{r}(c)=c+d_{1}, & \partial_{r}\left(d_{1}\right)=-d_{1}, \\
\sigma_{r}(c)=c+s_{1}, & \sigma_{r}\left(s_{1}\right)=-s_{1},
\end{array}
$$

where the remaining generators of (1.1) are unchanged.

(2) For an element $\Sigma(\omega)=(\alpha, \beta, \gamma, \delta, \theta)$ of $Z_{n}^{-(}(2 g+1 ; l, m)$, we have;

$$
\begin{aligned}
\Sigma\left(\omega \partial_{\gamma}\right) & =\left(\alpha, \beta, \gamma+\delta_{1},-\delta_{1}, \delta_{2}, \cdots, \delta_{l}, \boldsymbol{\theta}\right), \\
\Sigma\left(\omega \sigma_{r}\right) & =\left(\alpha, \beta, \gamma+\theta_{1}, \delta,-\theta_{1}, \theta_{2}, \cdots, \theta_{m}\right), \\
\Sigma\left(\omega \mathscr{Y}_{1}\right) & =\left(\alpha_{1},-\beta_{1}+2 \gamma, \alpha_{2}, \beta_{2}, \cdots, \alpha_{g}, \beta_{g}, \gamma, \delta, \theta\right) .
\end{aligned}
$$

Among these automorphisms, we have easily the following equations;

Proposition 2.3. (1) $\partial_{i} g=g \partial_{i}, \sigma_{i} g=g \sigma_{i}$, where $g$ is an automorphism induced by a homeomorphism in $\left\{\rho^{ \pm}, \rho_{1 i}, \mu_{1}^{ \pm}, \tau_{1}^{ \pm}, \theta_{12}^{ \pm}, \mathscr{Y}_{1}\right\}$,

(2) $\partial_{j} \sigma_{a, k}^{ \pm}=\sigma_{a, k}^{ \pm} \partial_{j}, \partial_{j} \sigma_{r, k}=\sigma_{r, k} \partial_{j}, \sigma_{k} \partial_{a, j}^{ \pm}=\partial_{a, j}^{ \pm} \sigma_{k}, \sigma_{k} \partial_{\gamma, j}=\partial_{r, j} \sigma_{k}$,

(3) $\partial_{i} \partial_{a, j}^{ \pm}=\partial_{a, j}^{ \pm} \partial_{i}(i \neq j), \partial_{i} \partial_{r, j}=\partial_{r, j} \partial_{i}(i \neq j)$,

(4) $\partial_{i} \partial_{j} \partial_{a, j}^{ \pm}=\partial_{a, i}^{ \pm} \partial_{i} \partial_{j}(i \neq j), \partial_{i} \partial_{j} \partial_{r, j}=\partial_{r, i} \partial_{i} \partial_{j}(i \neq j)$,

(5) $\sigma_{i} \sigma_{a, k}^{ \pm}=\sigma_{a, k}^{ \pm} \sigma_{i}(i \neq k), \sigma_{i} \sigma_{r, k}=\sigma_{r, k} \sigma_{i}(i \neq k)$,

(6) $\sigma_{i} \sigma_{k} \sigma_{a, k}^{ \pm}=\sigma_{a, i}^{ \pm} \sigma_{i} \sigma_{k}(i \neq k), \sigma_{i} \sigma_{k} \sigma_{r, k}=\sigma_{r, i} \sigma_{i} \sigma_{k}(i \neq k)$, where $\partial_{a, j}=\partial_{j} \partial_{a} \partial_{j}, \partial_{r, j}=\partial_{j} \partial_{\gamma} \partial_{j}, \sigma_{a, k}=\sigma_{k} \sigma_{a} \sigma_{k}$ and $\sigma_{r, k}=\sigma_{k} \sigma_{\gamma} \sigma_{k}$.

Then, we have the following;

LEMMA 2.2. (I) If $n$ is even and $d$ is even, any element $\left(\alpha_{1}, \beta_{1}\right.$, $\left.\alpha_{2}, \beta_{2}, \cdots, \alpha_{g}, \beta_{g}, \gamma, \delta_{1}, \delta_{2}, \cdots, \delta_{l}, \theta_{1}, \theta_{2}, \cdots, \theta_{m}\right)$ of $Z_{n}^{-}(g ; l, m)$ is equivalent to $\left(2,0,0,0, \cdots 0,0, \gamma, \delta_{1}, \delta_{2}, \cdots, \delta_{l}, \theta_{1}, \theta_{2}, \cdots, \theta_{m}\right)$, where $d=$ g.c.d. $\left\{\alpha_{1}, \beta_{1}\right.$, 
$\left.\alpha_{2}, \beta_{2}, \cdots, \alpha_{g}, \beta_{g}, \delta_{1}, \delta_{2}, \cdots, \delta_{l}, \theta_{1}, \theta_{2}, \cdots, \theta_{m}, n\right\}$

(II) If $d$ is odd, any element $\left(\alpha_{1}, \beta_{1}, \alpha_{2}, \beta_{2}, \cdots, \alpha_{g}, \beta_{g}, \gamma, \delta_{1}, \delta_{2}, \cdots, \delta_{l}\right.$, $\left.\theta_{1}, \theta_{2}, \cdots, \theta_{m}\right)$ of $Z_{n}^{-}(g ; l, m)$ is equivalent to $\left(1,0,0,0, \cdots, 0,0, \gamma, \delta_{1}, \delta_{2}, \cdots\right.$, $\left.\delta_{l}, \theta_{1}, \theta_{2}, \cdots, \theta_{m}\right)$.

Proof. By the same way as Lemmas 2 and 4 in [7], we have $\left(\alpha_{1}, \beta_{1}, \alpha_{2}, \beta_{2}, \cdots, \alpha_{g}, \beta_{g}, \gamma, \delta_{1}, \delta_{2}, \cdots, \delta_{l}, \theta_{1}, \theta_{2}, \cdots, \theta_{m}\right) \sim(d, 0,0,0, \cdots, 0,0$, $\left.\gamma, \delta_{1}, \delta_{2}, \cdots, \delta_{l}, \theta_{1}, \theta_{2}, \cdots, \theta_{m}\right)$. Since g.c.d. $\{d, \gamma\} \equiv 1(\bmod n)$, there are natural numbers $z$ and $z^{\prime}$ such that $z d+z^{\prime} \gamma \equiv 1(\bmod n)$. Let $h_{1}=$ $\mathscr{Y}_{1} \mu_{1} \tau_{1} \mu_{1}^{-1} \tau_{1}^{-z^{\prime}} \mathscr{Y}_{1} \tau_{1}^{-\left(z^{\prime}+1-2 z\right)}$ then $\omega \approx \omega h_{1} . \quad \Sigma\left(\omega h_{1}\right)=\left(2,-d, 0,0, \cdots, 0,0, \gamma, \delta_{1}\right.$, $\left.\delta_{2}, \cdots, \delta_{l}, \theta_{1}, \theta_{2}, \cdots, \theta_{m}\right)$ is equivalent to $\Sigma(\omega)=\left(d, 0,0,0, \cdots, 0,0, \gamma, \delta_{1}\right.$, $\left.\delta_{2}, \cdots, \delta_{l}, \theta_{1}, \theta_{2}, \cdots, \theta_{m}\right)$. If $d$ is odd, we take a homeomorphism $h_{2}=$ $\mu_{1} \tau_{1}^{(d+1) / 2} \mu_{1}^{-1} \tau_{1}^{2} \mu_{1}$. Then $\Sigma\left(\omega h_{1} h_{2}\right)=(1,0,0,0, \cdots, 0,0, \gamma, \delta, \theta)$ is equivalent to $\Sigma(\omega)$. If $d$ is even, we take a homeomorphism $h_{2}^{\prime}=\mu_{1} \tau_{1}^{d / 2} \mu_{1}^{-1}$. Then $\Sigma\left(\omega h_{1} h_{2}^{\prime}\right)=(2,0,0,0, \cdots, 0,0, \gamma, \delta, \theta)$, is equivalent to $\Sigma(\omega)$.

Lemma 2.3. Any element $\left(\alpha, 0, \cdots, 0, \gamma, \delta_{1}, \delta_{2}, \cdots, \delta_{l}, \theta_{1}, \theta_{2}, \cdots, \theta_{m}\right)$ with $\alpha=1$ or 2 , is equivalent to $\left(\alpha, 0, \cdots, 0, \gamma^{\prime}, \delta_{1}^{\prime}, \delta_{2}^{\prime}, \cdots, \delta_{l}^{\prime}, \theta_{1}^{\prime}, \theta_{2}^{\prime}, \cdots, \theta_{m}^{\prime}\right)$, where $0 \leqq \delta_{1}^{\prime} \leqq \delta_{2}^{\prime} \leqq \cdots \leqq \delta_{l}^{\prime} \leqq n / 2$ and $1 \leqq \theta_{1}^{\prime} \leqq \theta_{2}^{\prime} \leqq \cdots \leqq \theta_{m}^{\prime} \leqq n / 2$.

PRoof. If $\delta_{j}>n / 2$, we apply $\partial_{r, j}$. Then $\Sigma\left(\omega \partial_{r, j}\right)=\left(\alpha, 0, \cdots, 0, \gamma+\delta_{j}\right.$, $\left.\delta_{1}, \cdots, n-\delta_{j}, \cdots, \delta_{l}, \theta\right)$ is equivalent to $\Sigma(\omega)=\left(\alpha, 0, \cdots, 0, \gamma, \delta_{1}, \delta_{2}, \cdots, \delta_{l}\right.$, $\left.\theta_{1}, \theta_{2}, \cdots, \theta_{m}\right)$, where $n-\delta_{j}<n / 2$. Hence we have $\delta_{j} \leqq n / 2(1 \leqq j \leqq l)$. By the same way, we have $\theta_{k} \leqq n / 2(1 \leqq k \leqq m)$. Applying $\partial_{j}$ and $\sigma_{k}$ in a suitable way, we have $0 \leqq \delta_{1} \leqq \delta_{2} \leqq \cdots \leqq \delta_{l}$ and $1 \leqq \theta_{1} \leqq \theta_{2} \leqq \cdots \leqq \theta_{m}$.

LEMMA 2.4. If $\Sigma(\omega)=\left(\alpha_{1}, \beta_{1}, \cdots, \alpha_{g}, \beta_{g}, \gamma, \delta_{1}, \delta_{2}, \cdots, \delta_{l}, \theta_{1}, \theta_{2}, \cdots, \theta_{m}\right)$ and $\Sigma\left(\omega^{\prime}\right)=\left(\alpha_{1}^{\prime}, \beta_{1}^{\prime}, \cdots, \alpha_{g}^{\prime}, \beta_{g}^{\prime}, \gamma^{\prime}, \delta_{1}^{\prime}, \delta_{2}^{\prime}, \cdots, \delta_{l}^{\prime}, \theta_{1}^{\prime}, \theta_{2}^{\prime}, \cdots, \theta_{m}^{\prime}\right)$ are equivalent elements of $Z_{n}^{-}(2 g+1 ; l, m)$ satisfying the following conditions; (1) $0 \leqq$ $\delta_{1} \leqq \delta_{2} \leqq \cdots \leqq \delta_{l} \leqq n / 2,(2) 1 \leqq \theta_{1} \leqq \theta_{2} \leqq \cdots \leqq \theta_{m} \leqq n / 2,(3) 0 \leqq \delta_{1}^{\prime} \leqq \delta_{2}^{\prime} \leqq \cdots \leqq \delta_{l}^{\prime} \leqq n / 2$ and (4) $1 \leqq \theta_{1}^{\prime} \leqq \theta_{2}^{\prime} \leqq \cdots \leqq \theta_{m}^{\prime} \leqq n / 2$, then we have $\delta_{j}=\delta_{j}^{\prime}(1 \leqq j \leqq l)$ and $\theta_{k}=\theta_{k}^{\prime}$ $(1 \leqq k \leqq m)$.

Proof. Since $\Sigma(\omega) \sim \Sigma\left(\omega^{\prime}\right)$, by Proposition 2.2, there exists a homeomorphism $h$ of $(X, S)$ onto itself which is a composition of elements in $\left\{\rho^{ \pm}, \rho_{1 i}, \tau_{1}^{ \pm}, \mu_{1}^{ \pm}, \theta_{12}^{ \pm}, \partial_{j}, \sigma_{k}, \partial_{a}^{ \pm}, \sigma_{a}^{ \pm}, \partial_{r}, \sigma_{r}, \mathscr{Y}_{1}\right\}$ such that $\omega^{\prime}=\omega h_{*}$, where $h_{*}$ is the automorphism of $H_{1}(X-S)$ induced by $\left.h\right|_{x-s}$. By Lemma 2.1 and Lemmas 1 and 3 in [7], we note that for any $j(1 \leqq j \leqq l)$ there exists an integer $i(1 \leqq i \leqq l)$ such that $\delta_{j}=\delta_{i}^{\prime}$ or $\delta_{j}=n-\delta_{i}^{\prime}$. Since $0 \leqq \delta_{j}, \delta_{i}^{\prime} \leqq n / 2$, $0 \leqq \delta_{1} \leqq \delta_{2} \leqq \cdots \leqq \delta_{l} \leqq n / 2$ and $0 \leqq \delta_{1}^{\prime} \leqq \delta_{2}^{\prime} \leqq \cdots \leqq \delta_{l}^{\prime} \leqq n / 2$, we have $\delta_{j}=\delta_{j}^{\prime}(0 \leqq$ $j \leqq l)$. By the same way, we have $\theta_{k}=\theta_{k}^{\prime}(1 \leqq k \leqq m)$.

TheOREM 2.1. ( $n$; odd) A complete set of the equivalence classes of 
$Z_{n}^{-}(2 g+1 ; l, m)$ is given by;

(1)

$$
\check{\Sigma}_{n}^{-}(2 g+1 ; l, m)=\left\{\begin{array}{l}
\left(1,0, \cdots, 0,0, \gamma, \delta_{1}, \delta_{2}, \cdots, \delta_{l}, \theta_{1}, \theta_{2}, \cdots, \theta_{m}\right) ; \\
0 \leqq \delta_{1} \leqq \delta_{2} \leqq \cdots \leqq \delta_{l}<\frac{n}{2}, \\
1 \leqq \theta_{1} \leqq \theta_{2} \leqq \cdots \leqq \theta_{m}<\frac{n}{2}, \\
2 \gamma+\delta_{1}+\delta_{2}+\cdots+\delta_{l}+\theta_{1}+\theta_{2}+\cdots+\theta_{m} \equiv 0 \quad(\bmod n)
\end{array}\right\}
$$

$$
\begin{aligned}
\mathscr{Z}_{n}^{-}(1 ; l, m)=\left\{\begin{array}{l}
\left(\gamma, \delta_{1}, \delta_{2}, \cdots, \delta_{l}, \theta_{1}, \theta_{2}, \cdots, \theta_{m}\right) ; \\
0 \leqq \delta_{1} \leqq \delta_{2} \leqq \cdots \leqq \delta_{l}<\frac{n}{2}, \\
1 \leqq \theta_{1} \leqq \theta_{2} \leqq \cdots \leqq \theta_{m}<\frac{n}{2}, \\
2 \gamma+\delta_{1}+\delta_{2}+\cdots+\delta_{l}+\theta_{1}+\theta_{2}+\cdots+\theta_{m} \equiv 0 \quad(\bmod n) \\
\text { g.c.d. }\left\{\gamma, \delta_{1}, \delta_{2}, \cdots, \delta_{l}, \theta_{1}, \theta_{2}, \cdots, \theta_{m}, n\right\}=1
\end{array}\right\} \\
\text { if } g=0 .
\end{aligned}
$$

Proof. By Lemmas 2.2 and 2.3, any element $\Sigma(\omega)=(\alpha, \beta, \gamma, \delta, \theta)$ of $Z_{n}^{-}(2 g+1 ; l, m)$ is equivalent to an element of $\mathscr{Z}_{n}^{-}(2 g+1 ; l, m)$. Hence it is sufficient to prove that two distinct elements of $\mathscr{Z}_{n}^{-}(2 g+1 ; l, m)$ are not equivalent.

Let $\Sigma(\omega)=\left(1,0,0, \cdots, 0,0, \gamma, \delta_{1}, \delta_{2}, \cdots, \delta_{l}, \theta_{1}, \theta_{2}, \cdots, \theta_{m}\right)$ and $\Sigma\left(\omega^{\prime}\right)=$ $\left(1,0,0, \cdots, 0,0, \gamma^{\prime}, \delta_{1}^{\prime}, \delta_{2}^{\prime}, \cdots, \delta_{l}^{\prime}, \theta_{1}^{\prime}, \theta_{2}^{\prime}, \cdots, \theta_{m}^{\prime}\right)$ be equivalent elements of $\mathscr{Z}_{n}^{-}(2 g+1 ; l, m)$ where $g \geqq 1$. Then, by Lemma 2.4, we have $\delta_{j}=\delta_{j}^{\prime}(1 \leqq$ $j \leqq l)$ and $\theta_{k}=\theta_{k}^{\prime}(1 \leqq k \leqq m)$. Hence we have $2 \gamma^{\prime} \equiv-\left(\delta_{1}^{\prime}+\delta_{2}^{\prime}+\cdots+\delta_{l}^{\prime}+\theta_{1}^{\prime}+\right.$ $\left.\theta_{2}^{\prime}+\cdots+\theta_{m}^{\prime}\right)=-\left(\delta_{1}+\delta_{2}+\cdots+\delta_{l}+\theta_{1}+\theta_{2}+\cdots+\theta_{m}\right) \equiv 2 \gamma(\bmod n)$. Since $n$ is odd, we have $\gamma \equiv \gamma^{\prime}(\bmod n)$.

Let $\Sigma(\omega)=(\gamma, \delta, \theta)$ and $\Sigma\left(\omega^{\prime}\right)=\left(\gamma^{\prime}, \delta^{\prime}, \theta^{\prime}\right)$ be equivalent elements of $\mathscr{\varkappa}_{n}^{-}(1 ; l, m)$. By the same way, we have $(\gamma, \delta, \theta)=\left(\gamma^{\prime}, \delta^{\prime}, \boldsymbol{\theta}^{\prime}\right)$.

Now, we assume that $n$ is even. Suppose that an element $(\alpha, 0$, $0, \cdots, 0,0, \gamma, \delta_{1}, \delta_{2}, \cdots, \delta_{l}, \theta_{1}, \theta_{2}, \cdots, \theta_{m}$ ) satisfies the conditions of Lemma 2.3. If $n / 2 \leqq \gamma<n$ and $\delta_{l}=n / 2$ (or $\theta_{m}=n / 2$ ), then we apply $\partial_{r, l}$ (or $\sigma_{r, m}$ ). Then, we have the following;

LEMMA 2.5. ( $n$; even) If $n / 2 \leqq \gamma<n$ and $\delta_{l}=n / 2$ (or $\left.\theta_{m}=n / 2\right)$, then 
we have $\left(\alpha, 0,0,0, \cdots, 0,0, \gamma, \delta_{1}, \delta_{2}, \cdots, \delta_{l}, \theta_{1}, \theta_{2}, \cdots, \theta_{m}\right) \sim(\alpha, 0,0, \cdots, 0,0$, $\left.\gamma-n / 2, \delta_{1}, \delta_{2}, \cdots \delta_{l}, \theta_{1}, \theta_{2}, \cdots, \theta_{m}\right)$.

LEMMA 2.6. Suppose that $\Sigma(\omega)=\left(\alpha_{1}, \beta_{1}, \cdots, \alpha_{g}, \beta_{g}, \gamma, \delta_{1}, \delta_{2}, \cdots, \delta_{l}, \theta_{1}\right.$, $\left.\theta_{2}, \cdots, \theta_{m}\right)$ and $\Sigma\left(\omega^{\prime}\right)=\left(\alpha_{1}^{\prime}, \beta_{1}^{\prime}, \cdots, \alpha_{g}^{\prime}, \beta_{g}^{\prime}, \gamma^{\prime}, \delta_{1}^{\prime}, \delta_{2}^{\prime}, \cdots, \delta_{l}^{\prime}, \theta_{1}^{\prime}, \theta_{2}^{\prime}, \cdots, \theta_{m}^{\prime}\right)$ are

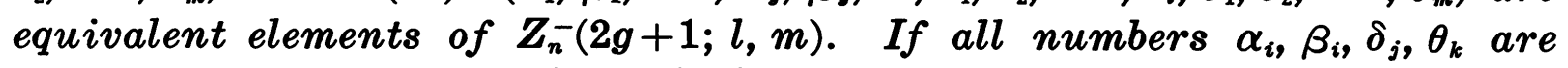
even, then all numbers $\alpha_{i}^{\prime}, \beta_{i}^{\prime}, \delta_{j}^{\prime}, \theta_{k}^{\prime}$ also are even.

Proof. Since $\Sigma(\omega) \sim \Sigma\left(\omega^{\prime}\right)$, by Proposition 2.2, there exists a homeomorphism $h$ of $(X, S)$ onto itself which is a composition of elements in $\left\{\rho^{ \pm}, \rho_{1 i}, \tau_{1}^{ \pm}, \mu_{1}^{ \pm}, \theta_{12}^{ \pm}, \partial_{j}, \sigma_{k}, \partial_{a}^{ \pm}, \sigma_{a}^{ \pm}, \partial_{r}, \sigma_{r}, \mathscr{Y}_{1}\right\}$ such that $\omega^{\prime}=\omega h_{*}$. If all numbers $\alpha_{i}, \beta_{i}, \delta_{j}, \theta_{k}$ are even, then all numbers $\alpha_{i}^{\prime}, \beta_{i}^{\prime}, \delta_{j}^{\prime}, \theta_{k}^{\prime}$ are even by Lemmas 1 and 3 in [7] and Lemma 2.1 .

THEOREM 2.2. ( $n$; even) A complete set of the equivalence classes of $Z_{n}^{-}(2 g+1 ; l, m)$ is given by the disjoint union $\mathscr{X}_{n}^{-}(2 g+1 ; l, m)$ of the following sets;

$$
\begin{aligned}
& \mathscr{\varkappa}_{n}^{-}(2 g+1 ; l, m)_{1}^{0}=\left\{\begin{array}{l}
\left(1,0, \cdots, 0,0, \gamma, \delta_{1}, \delta_{2}, \cdots, \delta_{l}, \theta_{1}, \theta_{2}, \cdots, \theta_{m}\right) ; \\
0 \leqq \delta_{1} \leqq \delta_{2} \leqq \cdots \leqq \delta_{l}<\frac{n}{2}, \\
1 \leqq \theta_{1} \leqq \theta_{2} \leqq \cdots \leqq \theta_{m}<\frac{n}{2}, \\
2 \gamma+\delta_{1}+\delta_{2}+\cdots+\delta_{l}+\theta_{1}+\theta_{2}+\cdots+\theta_{m} \equiv 0(\bmod n)
\end{array}\right\} \\
& \mathscr{Z}_{n}^{-}(2 g+1 ; l, m)_{1}^{*}=\left\{\begin{array}{l}
\left(1,0, \cdots, 0,0, \gamma, \delta_{1}, \delta_{2}, \cdots, \delta_{l}, \theta_{1}, \theta_{2}, \cdots, \theta_{m}\right) ; \\
\delta_{l}=\frac{n}{2} \text { or } \theta_{m}=\frac{n}{2}, 0 \leqq \gamma<\frac{n}{2}, \\
0 \leqq \delta_{1} \leqq \delta_{2} \leqq \cdots \leqq \delta_{l} \leqq \frac{n}{2}, \\
2 \gamma+\delta_{1} \leqq \theta_{2} \leqq \cdots \leqq \delta_{m} \leqq \frac{n}{2},
\end{array}\right\} \\
& \mathscr{\varkappa}_{n}^{-}(2 g+1 ; l, m)_{2}^{0}=\left\{\begin{array}{l}
\left(2,0, \cdots, 0,0, \gamma, \delta_{1}, \delta_{2}, \cdots, \delta_{l}, \theta_{1}, \theta_{2}, \cdots, \theta_{m}\right) ; \\
\gamma \text { is odd }, \delta_{j} \text { is even, } \theta_{k} \text { is even, } \\
0 \leqq \delta_{1} \leqq \delta_{2} \leqq \cdots \leqq \delta_{l}<\frac{n}{2}, \\
1 \leqq \theta_{1} \leqq \theta_{2} \leqq \cdots \leqq \theta_{m}<\frac{n}{2}, \\
2 \gamma+\delta_{1}+\delta_{2}+\cdots+\delta_{l}+\theta_{1}+\theta_{2}+\cdots+\theta_{m} \equiv 0(\bmod n)
\end{array}\right\}
\end{aligned}
$$


$\mathscr{\varkappa}_{n}^{-}(2 g+1 ; l, m)_{2}^{*}=\left\{\begin{array}{c}\left(2,0, \cdots, 0,0, \gamma, \delta_{1}, \delta_{2}, \cdots, \delta_{l}, \theta_{1}, \theta_{2}, \cdots, \theta_{m}\right) ; \\ \delta_{l}=\frac{n}{2} \text { or } \theta_{m}=\frac{n}{2}, 0 \leqq \gamma<\frac{n}{2}, \\ \gamma \text { is odd, } \delta_{j} \text { is even, } \theta_{k} \text { is even }, \\ 0 \leqq \delta_{1} \leqq \delta_{2} \leqq \cdots \leqq \delta_{l} \leqq \frac{n}{2}, \\ 1 \leqq \theta_{1} \leqq \theta_{2} \leqq \cdots \leqq \theta_{m} \leqq \frac{n}{2}, \\ 2 \gamma+\delta_{1}+\delta_{2}+\cdots+\delta_{l}+\theta_{1}+\theta_{2}+\cdots+\theta_{m} \equiv 0 \quad(\bmod n)\end{array}\right\}$

( 2 )

$$
\begin{aligned}
& \mathscr{Z}_{n}^{-}(1 ; l, m)^{0}=\left\{\begin{array}{l}
\left(\gamma, \delta_{1}, \delta_{2}, \cdots, \delta_{l}, \theta_{1}, \theta_{2}, \cdots, \theta_{m}\right) ; \\
0 \leqq \delta_{1} \leqq \delta_{2} \leqq \cdots \leqq \delta_{l}<\frac{n}{2}, \\
1 \leqq \theta_{1} \leqq \theta_{2} \leqq \cdots \leqq \theta_{m}<\frac{n}{2}, \\
2 \gamma+\delta_{1}+\delta_{2}+\cdots+\delta_{l}+\theta_{1}+\theta_{2}+\cdots+\theta_{m} \equiv 0(\bmod n), \\
\text { g.c.d. }\left\{\gamma, \delta_{1}, \delta_{2}, \cdots, \delta_{l}, \theta_{1}, \theta_{2}, \cdots, \theta_{m}, n\right\}=1
\end{array}\right) \\
& \mathscr{\varkappa}_{n}^{-}(1 ; l, m)^{*}=\left\{\begin{array}{l}
\left(\gamma, \delta_{1}, \delta_{2}, \cdots, \delta_{l}, \theta_{1}, \theta_{2}, \cdots, \theta_{m}\right) ; \\
\delta_{l}=\frac{n}{2} \quad o r \quad \theta_{m}=\frac{n}{2}, 0 \leqq \gamma<\frac{n}{2}, \\
0 \leqq \delta_{1} \leqq \delta_{2} \leqq \cdots \leqq \delta_{l} \leqq \frac{n}{2}, \\
1 \leqq \theta_{1} \leqq \theta_{2} \leqq \cdots \leqq \theta_{m} \leqq \frac{n}{2}, \\
2 \gamma+\delta_{1}+\delta_{2}+\cdots+\delta_{l}+\theta_{1}+\theta_{2}+\cdots+\theta_{m} \equiv 0(\bmod n), \\
\mathrm{g} . \mathrm{c} . \mathrm{d} .\left\{\gamma, \delta_{1}, \delta_{2}, \cdots, \delta_{l}, \theta_{1}, \theta_{2}, \cdots, \theta_{m}, n\right\}=1 \\
g=0 .
\end{array}\right.
\end{aligned}
$$

Proof of Theorem 2.2(1). By Lemmas 2.2, 2.3 and 2.5, any element $\Sigma(\omega)=(\alpha, \beta, \gamma, \delta, \theta)$ of $Z_{n}^{-}(2 g+1 ; l, m)$ is equivalent to an element of $\mathscr{\varkappa}_{n}^{-}(2 g+1 ; l, m)$. Hence it is sufficient to prove that two distinct elements of $\mathscr{\varkappa}_{n}^{-}(2 g+1 ; l, m)$ are not equivalent. We will prove it in respective cases.

(i) Let $\Sigma(\omega)=\left(\alpha, 0, \cdots, 0,0, \gamma, \delta_{1}, \delta_{2}, \cdots, \delta_{l}, \theta_{1}, \theta_{2}, \cdots, \theta_{m}\right)$ be an element of $\mathscr{\varkappa}_{n}^{-}(2 g+1 ; l, m)_{\alpha}^{*}$ and $\Sigma\left(\omega^{\prime}\right)=\left(\alpha^{\prime}, 0, \cdots, 0,0, \gamma^{\prime}, \delta_{1}^{\prime}, \delta_{2}^{\prime}, \cdots, \delta_{l}^{\prime}, \theta_{1}^{\prime}, \theta_{2}^{\prime}\right.$, $\left.\cdots, \theta_{m}^{\prime}\right)$ be an element of $\mathscr{\varkappa}_{n}^{-}(2 g+1 ; l, m)_{\alpha^{\prime}}^{0}$, where $\alpha=1$ or 2 and $\alpha^{\prime}=1$ 
or 2. By Lemma 2.4, it is impossible that $\Sigma(\omega)$ and $\Sigma\left(\omega^{\prime}\right)$ are equivalent.

(ii) Let $\Sigma(\omega)=\left(2,0, \cdots, 0,0, \gamma, \delta_{1}, \delta_{2}, \cdots, \delta_{l}, \theta_{1}, \theta_{2}, \cdots, \theta_{m}\right)$ and $\Sigma\left(\omega^{\prime}\right)=$ $\left(1,0, \cdots, 0,0, \gamma^{\prime}, \delta_{1}^{\prime}, \delta_{2}^{\prime}, \cdots, \delta_{l}^{\prime}, \theta_{1}^{\prime}, \theta_{2}^{\prime}, \cdots, \theta_{m}^{\prime}\right)$ be equivalent. Since all numbers $\delta_{j}, \theta_{k}$ are even, it is impossible by Lemma 2.6.

(iii) Let $\Sigma(\omega)=\left(\alpha, 0, \cdots, 0,0, \gamma, \delta_{1}, \delta_{2}, \cdots, \delta_{l}, \theta_{1}, \theta_{2}, \cdots, \theta_{m}\right)$ and $\Sigma\left(\omega^{\prime}\right)=$ $\left(\alpha, 0, \cdots, 0,0, \gamma^{\prime}, \delta_{1}^{\prime}, \delta_{2}^{\prime}, \cdots, \delta_{l}^{\prime}, \theta_{1}^{\prime}, \theta_{2}^{\prime}, \cdots, \theta_{m}^{\prime}\right)$ be equivalent elements of $\mathscr{Z}_{n}^{-}(2 g+1 ; l, m)_{\alpha}^{*}$, where $\alpha=1$ or 2 . By Lemma 2.4 , we have $\delta_{j}=\delta_{j}^{\prime}$ $(1 \leqq j \leqq l)$ and $\theta_{k}=\theta_{k}^{\prime}(1 \leqq k \leqq m)$. Hence we have $2 \gamma^{\prime} \equiv-\left(\delta_{1}^{\prime}+\delta_{2}^{\prime}+\cdots+\delta_{l}^{\prime}+\right.$ $\left.\theta_{1}^{\prime}+\theta_{2}^{\prime}+\cdots+\theta_{m}^{\prime}\right)=-\left(\delta_{1}+\delta_{2}+\cdots+\delta_{l}+\theta_{1}+\theta_{2}+\cdots+\theta_{m}\right) \equiv 2 \gamma(\bmod n)$. Therefore we have $\gamma-\gamma^{\prime} \equiv 0(\bmod n / 2)$. Since $0 \leqq \gamma, \gamma^{\prime}<n / 2$, we have $\gamma=\gamma^{\prime}$.

(iv) Let $\Sigma(\omega)=\left(\alpha, 0, \cdots, 0,0, \gamma, \delta_{1}, \delta_{2}, \cdots, \delta_{l}, \theta_{1}, \theta_{2}, \cdots, \theta_{m}\right)$ and $\Sigma\left(\omega^{\prime}\right)=$ $\left(\alpha, 0, \cdots, 0,0, \gamma^{\prime}, \delta_{1}^{\prime}, \delta_{2}^{\prime}, \cdots, \delta_{l}^{\prime}, \theta_{1}^{\prime}, \theta_{2}^{\prime}, \cdots, \theta_{m}^{\prime}\right)$ be equivalent elements of $\mathscr{Z}_{n}^{-}(2 g+1 ; l, m)_{\alpha}^{0}$, where $\alpha=1$ or 2 . Then, there exists a homeomorphism $h$ of $(X, S)$ onto itself which is a composition of elements in $\left\{\rho^{ \pm}, \rho_{11}, \tau_{1}^{ \pm}\right.$, $\left.\boldsymbol{u}_{1}^{ \pm}, \theta_{12}^{ \pm}, \partial_{j}, \sigma_{k}, \partial_{a}^{ \pm}, \sigma_{a}^{ \pm}, \partial_{\gamma}, \sigma_{\gamma}, \mathscr{Y}_{1}\right\}$ such that $\omega^{\prime}=\omega h_{*}$. By Lemma 2.4, we have $\delta_{j}=\delta_{j}^{\prime}(1 \leqq j \leqq l)$ and $\theta_{k}=\theta_{k}^{\prime}(1 \leqq k \leqq m)$. Now, it is sufficient to prove the following lemma;

\section{LEMMA 2.7. $\gamma=\gamma^{\prime}$.}

Proof. By Proposition 2.3, we may assume $h=g \cdot g^{\prime}$ where $g^{\prime}$ is an automorphism induced by a composition of homeomorphisms in $\left\{\partial_{j}, \sigma_{k}\right\}$ and $g$ is an automorphism induced by a composition of homeomorphisms in $\left\{\rho^{ \pm}, \rho_{1 i}, \tau_{1}^{ \pm}, \mu_{1}^{ \pm}, \theta_{12}^{ \pm}, \partial_{a, j}^{ \pm}, \partial_{r, j}, \sigma_{a, k}^{ \pm}, \sigma_{r, k}, \mathscr{Y}_{1}\right\}$. We note that only an automorphism $\partial_{r, j}$ (resp. $\sigma_{\gamma, k}$ ) can change $\gamma$. Let $g=g_{i_{0}} g_{j_{1}} g_{i_{1}} g_{k_{1}} g_{i_{2}} g_{j_{2}} g_{i_{3}} g_{k_{2}} g_{i_{4}} \cdots$ $g_{i_{2 t-2}} g_{j_{t}} g_{i_{2 t-1}} g_{k_{t}} g_{i_{2 t}} \cdots g_{i_{2 s-2}} g_{j_{g}} g_{i_{2 s-1}-1} g_{k_{s}} g_{i_{2 g}}$, where $g_{j_{t}}$ is an automorphism $\partial_{r, j_{t}}$ or the identity $(1 \leqq t \leqq s), g_{k_{t}}$ is an automorphism $\sigma_{r, k_{t}}$ or the identity $(1 \leqq t \leqq s)$ and $g_{t_{t}}$ is an automorphism induced by a composition of homeomorphisms in $\left\{\rho^{ \pm}, \rho_{1 i}, \tau_{1}^{ \pm}, \mu_{1}^{ \pm}, \theta_{12}^{ \pm}, \partial_{a, j}^{ \pm}, \sigma_{a, k}^{ \pm}, \mathscr{Y}_{1}\right\}$ or the identity $(0 \leqq t \leqq 2 s)$.

We denote by $G(t)$ the number of elements of the set $\left\{u ; g_{j_{u}}=\partial_{r, j_{t}}\right.$ $(1 \leqq u \leqq t)\}$ and by $G_{*}(t)$ the number of elements of the set $\left\{u ; g_{k_{u}}=\sigma_{r, k_{t}}\right.$ $(1 \leqq u \leqq t)\}$. Then for any $t(1 \leqq t \leqq s)$, we have the following;

$$
\omega g_{i_{0}} g_{j_{1}} g_{i_{1}} g_{k_{1}} \cdots g_{i_{2 t-2}} g_{j_{t}}(c)=\gamma+\varepsilon_{1}^{\prime} \delta_{j_{1}}+\varepsilon_{2}^{\prime} \delta_{k_{1}}+\cdots+\varepsilon_{2 t-8}^{\prime} \delta_{j_{t-1}}+\varepsilon_{2 t-2}^{\prime} \theta_{j_{t-1}}+\varepsilon_{2 t-1}^{\prime} \theta_{j_{t}}
$$

and

$$
\omega g_{i_{0}} g_{j_{1}} g_{i_{1}} g_{k_{1}} \cdots g_{i_{2 t-2}} g_{j_{t}}\left(d_{j_{t}}\right)=\varepsilon_{2 t-1} \delta_{j_{t}},
$$

where

$$
\begin{aligned}
& \varepsilon_{2 t-1}^{\prime}=+1 \text { and } \varepsilon_{2 t-1}=-1 \text { if } g_{j_{t}}=\partial_{r, j_{t}} \text { and } G(t) \text { is odd, } \\
& \varepsilon_{2 t-1}^{\prime}=-1 \text { and } \varepsilon_{2 t-1}=+1 \text { if } g_{j_{t}}=\partial_{r, j_{t}} \text { and } G(t) \text { is even, } \\
& \varepsilon_{2 t-1}^{\prime}=0 \text { and } \varepsilon_{2 t-1}=-1 \text { if } g_{j_{t}} \text { is the identity and } G(t) \text { is odd, }
\end{aligned}
$$


$\varepsilon_{2 t-1}^{\prime}=0$ and $\varepsilon_{2 t-1}=+1$ if $g_{j_{t}}$ is the identity and $G(t)$ is even .

Moreover, for any $t(1 \leqq t \leqq s)$, we have the following;

$$
\begin{aligned}
& \omega g_{i_{0}} g_{j_{1}} g_{i_{1}} g_{k_{1}} \cdots g_{i_{2 t-2}} g_{i_{t}} g_{i_{2 t-1}} g_{k_{t}}(c)=\gamma+\varepsilon_{1}^{\prime} \delta_{j_{1}}+\varepsilon_{2}^{\prime} \theta_{k_{1}}+\cdots+\varepsilon_{2 t-1}^{\prime} \delta_{j_{t}}+\varepsilon_{2 t}^{\prime} \theta_{k_{t}} \quad \text { and } \\
& \omega g_{i_{0}} g_{j_{1}} g_{i_{1}} g_{k_{1}} \cdots g_{i_{2 t-2}} g_{i_{t}} g_{i_{2 t-1}} g_{k_{t}}\left(\varepsilon_{k_{t}}\right)=\varepsilon_{2 t} \theta_{k_{t}},
\end{aligned}
$$

where

$\varepsilon_{2 t}^{\prime}=+1$ and $\varepsilon_{2 t}=-1$ if $g_{k_{t}}=\sigma_{\gamma, k_{t}}$ and $G_{*}(t)$ is odd ,

$\varepsilon_{2 t}^{\prime}=-1$ and $\varepsilon_{2 t}=+1$ if $g_{k_{t}}=\sigma_{r, k_{t}}$ and $G_{*}(t)$ is even,

$\varepsilon_{2 t}^{\prime}=0$ and $\varepsilon_{2 t}=-1$ if $g_{k_{t}}$ is the identity and $G_{*}(t)$ is odd,

$\varepsilon_{2 t}^{\prime}=0$ and $\varepsilon_{2 t}=+1$ if $g_{k_{t}}$ is the identity and $G_{*}(t)$ is even .

If $0=\delta_{1}=\delta_{2}=\cdots=\delta_{l_{0}}<\delta_{l_{0}+1} \leqq \delta_{l_{0}+2} \leqq \cdots \leqq \delta_{l}<n / 2$ then the number $G_{j}$ of elements of the set $\left\{t ; g_{j_{t}}=\partial_{r, j}(0 \leqq t \leqq s)\right\}$ is even for any $j\left(l_{0}+1 \leqq j \leqq l\right)$. Because if there is an integer $j_{0}\left(l_{0}+1 \leqq j_{0} \leqq l\right)$ such that $G_{j_{0}}$ is odd, $\omega g_{i_{0}} g_{j_{1}} g_{i_{1}} g_{k_{1}} \cdots g_{i_{2 s-2}} g_{j_{s}} g_{i_{2 s-1}} g_{k_{s}} g_{i_{2 s}}\left(d_{j}\right)=-\delta_{j}$. Hence we have $\omega h_{*}\left(d_{j}\right)=n-\delta_{j}$ which is impossible. By the same way, the number $G_{k}^{*}$ of elements of the set $\left\{t ; g_{k_{t}}=\sigma_{r, k}(0 \leqq t \leqq s)\right\}$ is even for any $k(1 \leqq k \leqq m)$. Hence we have $\varepsilon_{1}^{\prime} \delta_{j_{1}}+\varepsilon_{2}^{\prime} \theta_{k_{1}}+\cdots+\varepsilon_{2 s-1}^{\prime} \delta_{j_{s}}+\varepsilon_{2 s}^{\prime} \theta_{k_{s}} \equiv 0(\bmod n)$. Therefore we have $\gamma^{\prime}=$ $\omega h_{*}(c)=\gamma+\varepsilon_{1}^{\prime} \delta_{j_{1}}+\varepsilon_{2}^{\prime} \theta_{k_{1}}+\cdots+\varepsilon_{2 s-1}^{\prime} \delta_{j_{s}}+\varepsilon_{2 s}^{\prime} \theta_{k_{s}} \equiv \gamma(\bmod n)$. This completes the proof of Lemma 2.7 .

Proof of TheOREM 2.2(2). It is sufficient to prove that two distinct elements of $\mathscr{\gamma}_{n}^{-}(1 ; l, m)$ are not equivalent. Let $\Sigma(\omega)=(\gamma, \delta, \theta)$ and $\Sigma\left(\omega^{\prime}\right)=\left(\gamma^{\prime}, \delta^{\prime}, \theta^{\prime}\right)$ be equivalent elements of $\tilde{\varkappa}_{n}^{-}(1 ; l, m)$. By the same way, we have $(\gamma, \delta, \theta)=\left(\gamma^{\prime}, \delta^{\prime}, \theta^{\prime}\right)$.

Using the homology group of a covering space of $X$ or by a geometric consideration, we have the following;

Proposition 2.4. (1) An element $(f, M)$ of $P_{n}$ corresponding to an element of $\tilde{\varkappa}_{n}^{-}(2 g+1 ; l, m)$ belongs to $P_{n}^{0}$, if $n$ is odd.

(2) An element $(f, M)$ of $P_{n}$ corresponding to an element of $\mathscr{\gamma}_{n}^{-}(2 g+1 ; l, m)_{1}^{0}$ or $\mathscr{Z}_{n}^{-}(2 g+1 ; l, m)_{1}^{*}$ belongs to $P_{n}^{0}$, if $n$ is even and $g \geqq 1$.

(3) An element $(f, M)$ of $P_{n}$ corresponding to an element of $\mathscr{\varkappa}_{n}^{-}(2 g+1 ; l, m)_{2}^{0}$ or $\mathscr{Y}_{n}^{-}(2 g+1 ; l, m)_{2}^{*}$ belongs to $P_{n}^{-}$, if $n$ is even and $g \geqq 1$.

(4) An element $(f, M)$ of $P_{n}$ corresponding to an element of $\mathscr{\varkappa}_{n}^{-}(1 ; l, m)^{0}$ or $\mathscr{Y}_{n}^{-}(1 ; l, m)^{*}$ belongs to $P_{n}^{0}$, if $n$ is even and $d$ is odd.

(5) An element $(f, M)$ of $P_{n}$ corresponding to an element of $\mathscr{Z}_{n}^{-}(1 ; l, m)^{0}$ or $\mathscr{K}_{n}^{-}(1 ; l, m)^{*}$ belongs to $P_{n}^{-}$, if $n$ is even and $d$ is even. Here $d=$ g.c.d. $\left\{\delta_{1}, \delta_{2}, \cdots, \delta_{l}, \theta_{1}, \theta_{2}, \cdots, \theta_{m}, n\right\}$. 
§ 3. Determination of the equivalence classes of $P_{n}\left(X_{2 g+2}, S\right), g \neq 0$.

In this section, we merely denote $X_{2 g+2}$ by $X$. To dentermine the equivalence classes of $P_{n}(X, S)$, we use the following result of Lickorish [3] and Chillingworth [2].
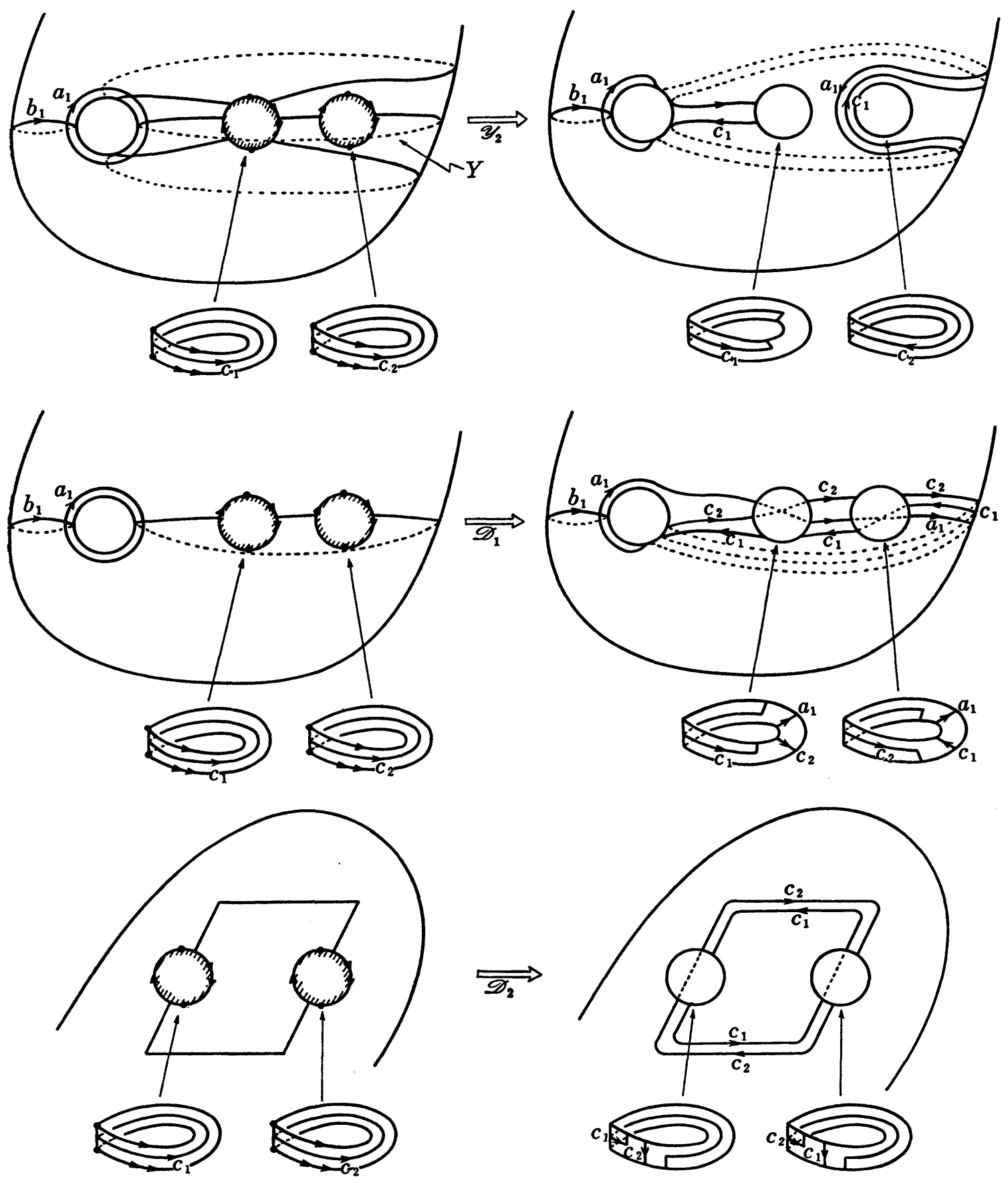

Figure 6 
Proposition 3.1. There exist a Y-homeomorphism $\mathscr{Y}_{2}$, Dehn twists $\mathscr{D}_{1}$ and $\mathscr{D}_{2}$ of $(X, S)$ onto itself such that the automorphisms of $H_{1}(X-S)$ induced by them are given by;

$$
\begin{aligned}
& \mathscr{Y}_{2}\left(a_{1}\right)=a_{1}-2 c_{2}, \quad \mathscr{Y}_{2}\left(b_{1}\right)=b_{1}, \quad \mathscr{Y}_{2}\left(c_{1}\right)=c_{1}+2 c_{2}, \quad \mathscr{Y}_{2}\left(c_{2}\right)=-c_{2}, \\
& \mathscr{D}_{1}\left(a_{1}\right)=a_{1}-b_{1}+c_{1}+c_{2}, \quad \mathscr{D}_{1}\left(b_{1}\right)=b_{1}, \quad \mathscr{D}_{1}\left(c_{1}\right)=b_{1}-c_{2}, \\
& \mathscr{D}_{1}\left(c_{2}\right)=-b_{1}+c_{1}+2 c_{2}, \\
& \mathscr{D}_{2}\left(c_{1}\right)=-c_{2}, \quad \mathscr{D}_{2}\left(c_{2}\right)=c_{1}+2 c_{2},
\end{aligned}
$$

where the remaining generators of (1.2) are unchanged, see Fig. 6.

REMARK. These homeomorphisms $\mathscr{D}_{1}$ and $\mathscr{D}_{2}$ are the same maps as twists about the curves $\gamma_{N}$ and $\beta_{N+1}$ in Chillingworth [2].

By the same way as in $\S 2$, we define some typical homeomorphisms of surfaces.

Definition 3.1. (1) $\partial_{\gamma_{1}}, \partial_{\gamma_{2}}$ : In parallel to definition of $\partial_{r}$ in Definition 2.1, we define homeomorphisms $\partial_{r_{1}}$ and $\partial_{\gamma_{2}}$ corresponding to the two Möbius bands in Fig. 2.

(2) $\sigma_{\tau_{1}}, \sigma_{\gamma_{2}}$ : In parallel to definition of $\sigma_{r}$ in Definition 2.1, we define homeomorphism $\sigma_{r_{1}}$ and $\sigma_{r_{2}}$ corresponding to the two Möbius bands in Fig. 2.

Then, by Suzuki [5] and Chillingworth [2] and in an elementary way, we have the following;

Proposition 3.2. The homeotopy group of $(X, S)$ is generated by $\rho, \rho_{1 i}(2 \leqq i \leqq g), \tau_{1}, \mu_{1}, \theta_{12}, \mathscr{Y}_{2}, \mathscr{D}_{1}, \mathscr{D}_{2}, \partial_{j}(2 \leqq j \leqq l), \sigma_{k}(2 \leqq k \leqq m), \partial_{a}, \sigma_{a}, \partial_{r_{1}}$, $\partial_{r_{2}}, \sigma_{r_{1}}$ and $\sigma_{r_{2}}$.

LEMMA 3.1. (1) The automorphisms of $H_{1}(X-S)$ induced by them are given by;

$$
\begin{array}{ll}
\partial_{r_{1}}\left(c_{1}\right)=c_{1}+d_{1}, & \partial_{r_{1}}\left(d_{1}\right)=-d_{1} ; \\
\partial_{r_{2}}\left(c_{2}\right)=c_{2}+d_{1}, & \partial_{r_{2}}\left(d_{1}\right)=-d_{1} ; \\
\sigma_{r_{1}}\left(c_{1}\right)=c_{1}+s_{1}, & \sigma_{r_{1}}\left(s_{1}\right)=-s_{1} ; \\
\sigma_{r_{2}}\left(c_{2}\right)=c_{2}+s_{1}, & \sigma_{r_{2}}\left(s_{1}\right)=-s_{1} ;
\end{array}
$$

where the remaining generators of (1.2) are unchanged.

(2) For an element $\Sigma(\omega)=\left(\alpha, \beta, \gamma_{1}, \gamma_{2}, \delta, \theta\right)$ of $Z_{n}^{-}(2 g+2 ; l, m)$, we have

$$
\begin{aligned}
& \Sigma\left(\omega \partial_{\gamma_{1}}\right)=\left(\alpha, \beta, \gamma_{1}+\delta_{1}, \gamma_{2},-\delta_{1}, \delta_{2}, \cdots, \delta_{l}, \theta\right), \\
& \Sigma\left(\omega \partial_{\gamma_{2}}\right)=\left(\alpha, \beta, \gamma_{1}, \gamma_{2}+\delta_{1},-\delta_{1}, \delta_{2}, \cdots, \delta_{l}, \theta\right),
\end{aligned}
$$




$$
\begin{aligned}
& \Sigma\left(\omega \sigma_{\gamma_{1}}\right)=\left(\alpha, \beta, \gamma_{1}+\theta_{1}, \gamma_{2}, \delta,-\theta_{1}, \theta_{2}, \cdots, \theta_{m}\right), \\
& \Sigma\left(\omega \sigma_{\gamma_{2}}\right)=\left(\alpha, \beta, \gamma_{1}, \gamma_{2}+\theta_{1}, \delta,-\theta_{1}, \theta_{2}, \cdots, \theta_{m}\right), \\
& \Sigma\left(\omega \mathscr{Y}_{2}\right)=\left(\alpha_{1}-2 \gamma_{2}, \beta_{1}, \alpha_{2}, \beta_{2}, \cdots, \alpha_{g}, \beta_{g}, \gamma_{1}+2 \gamma_{2},-\gamma_{2}, \delta, \theta\right), \\
& \Sigma\left(\omega \mathscr{D}_{1}\right)=\left(\alpha_{1}-\beta_{1}+\gamma_{1}+\gamma_{2}, \beta_{1}, \alpha_{2}, \beta_{2}, \cdots, \alpha_{g}, \beta_{g}, \beta_{1}-\gamma_{2},-\beta_{1}+\gamma_{1}+2 \gamma_{2}, \delta, \theta\right), \\
& \Sigma\left(\omega \mathscr{D}_{2}\right)=\left(\alpha, \beta,-\gamma_{2}, \gamma_{1}+2 \gamma_{2}, \delta, \theta\right) .
\end{aligned}
$$

Among these automorphisms, we have easily the following equations;

Proposition 3.3. (1) $\partial_{i} g=g \partial_{i}, \sigma_{i} g=g \sigma_{i}$, where $g$ is an automorphism induced by a homeomorphism in $\left\{\rho^{ \pm}, \rho_{1 i}, \mu_{1}^{ \pm}, \tau_{1}^{ \pm}, \theta_{12}^{ \pm}, \mathscr{Y}_{2}, \mathscr{D}_{1}^{ \pm}, \mathscr{D}_{2}^{ \pm}\right\}$,

(2) $\partial_{i} \sigma_{a, k}^{ \pm}=\sigma_{a, k}^{ \pm} \partial_{i}, \partial_{i} \sigma_{1, k}=\sigma_{1, k} \partial_{i}, \partial_{i} \sigma_{2, k}=\sigma_{2, k} \partial_{i}, \sigma_{i} \partial_{a, j}^{ \pm}=\partial_{a, j}^{ \pm} \sigma_{i}, \sigma_{i} \partial_{1, j}=\partial_{1, j} \sigma_{i}$, $\sigma_{i} \partial_{2, j}=\partial_{2, j} \sigma_{i}$

(3) $\partial_{i} \partial_{a, j}^{ \pm}=\partial_{a, j}^{ \pm} \partial_{i}(i \neq j), \partial_{i} \partial_{1, j}=\partial_{1, j} \partial_{i}(i \neq j), \partial_{i} \partial_{2, j}=\partial_{2, j} \partial_{i}(i \neq j)$,

(4) $\partial_{i} \partial_{j} \partial_{a, j}^{ \pm}=\partial_{a, i}^{ \pm} \partial_{i} \partial_{j}(i \neq j), \partial_{i} \partial_{j} \partial_{1, j}=\partial_{1, i} \partial_{i} \partial_{j}(i \neq j), \partial_{i} \partial_{j} \partial_{2, j}=\partial_{2, i} \partial_{i} \partial_{j}(i \neq j)$,

(5) $\sigma_{i} \sigma_{a, k}^{ \pm}=\sigma_{a, k}^{ \pm} \sigma_{i}(i \neq k), \sigma_{i} \sigma_{1, k}=\sigma_{1, k} \sigma_{i}(i \neq k), \sigma_{i} \sigma_{2, k}=\sigma_{2, k} \sigma_{i}(i \neq k)$, $(i \neq k)$,

(6) $\quad \sigma_{i} \sigma_{k} \sigma_{a, k}^{ \pm}=\sigma_{a, i}^{ \pm} \sigma_{i} \sigma_{k}(i \neq k), \sigma_{i} \sigma_{k} \sigma_{1, k}=\sigma_{1, i} \sigma_{i} \sigma_{k}(i \neq k), \sigma_{i} \sigma_{k} \sigma_{2, k}=\sigma_{2, i} \sigma_{i} \sigma_{k}$

where $\partial_{a, j}=\partial_{j} \partial_{a} \partial_{j}, \partial_{1, j}=\partial_{j} \partial_{\gamma_{1}} \partial_{j}, \partial_{2, j}=\partial_{j} \partial_{\gamma_{2}} \partial_{j}, \sigma_{a, k}=\sigma_{k} \sigma_{a} \sigma_{k}, \sigma_{1, k}=\sigma_{k} \sigma_{r_{1}} \sigma_{k}$ and $\sigma_{2, k}=\sigma_{k} \sigma_{r_{2}} \sigma_{k}$.

By the these results, we have the following;

LEMMA 3.2. (I) If $n$ is even, $d$ is even and $\gamma$ is even, then any element $\left(\alpha_{1}, \beta_{1}, \alpha_{2}, \beta_{2}, \cdots, \alpha_{g}, \beta_{g}, \gamma_{1}, \gamma_{2}, \delta, \theta\right)$ of $Z_{n}^{-}(2 g+2 ; l, m)$ is equivalent to $(2,0,0,0, \cdots, 0,0,1, \gamma-1, \delta, \theta)$, where $d=$ g.c.d. $\left\{\alpha_{1}, \beta_{1}, \alpha_{2}, \beta_{2}, \cdots, \alpha_{g}, \beta_{g}\right.$, $\left.\delta_{1}, \delta_{2}, \cdots, \delta_{l}, \theta_{1}, \theta_{2}, \cdots, \theta_{m}, n\right\}$ and $\gamma \equiv \gamma_{1}+\gamma_{2}(\bmod n)$.

(II) Otherwise, any element $\left(\alpha_{1}, \beta_{1}, \alpha_{2}, \beta_{2}, \cdots, \alpha_{g}, \beta_{g}, \gamma_{1}, \gamma_{2}, \delta, \theta\right)$ of $Z_{n}^{-}(2 g+2 ; l, m)$ is equivalent to $(1,0,0,0, \cdots, 0,0,0, \gamma, \delta, \theta)$.

Proof. By the same way as Lemmas 2 and 4 in [7], we have $\left(\alpha_{1}, \beta_{1}, \alpha_{2}, \beta_{2}, \cdots, \alpha_{g}, \beta_{g}, \gamma_{1}, \gamma_{2}, \delta, \theta\right) \sim\left(d, 0,0,0, \cdots, 0,0, \gamma_{1}, \gamma_{2}, \delta, \theta\right)$. Since g.c.d. $\left\{d, \gamma_{1}, \gamma_{2}\right\} \equiv 1(\bmod n)$, there are natural numbers $z_{0}, z_{1}$ and $z_{2}$ such that $z_{0} d+z_{1} \gamma_{1}+z_{2} \gamma_{2} \equiv 1(\bmod n)$. Let

$$
h_{1}=\mu_{1} \mathscr{D}_{2}^{-1} \mathscr{Y}_{2} \tau_{1} \mu_{1} \tau_{1}^{z_{1}-z_{2}} \mu_{1}^{-1} \mathscr{Y}_{2} \mathscr{D}_{2} \mu_{1}\left(\mathscr{D}_{2}^{-1} \mathscr{D}_{1}\right)^{2 \varepsilon_{2}} \tau_{1}^{z_{0}+1-z_{1}-z_{2}} \text {. }
$$

Then $\Sigma\left(\omega h_{1}\right)=\left(2,-d, 0,0, \cdots, 0,0,-2 z_{2} d+\gamma_{1}, 2 z_{2} d+\gamma_{2}, \delta, \theta\right)$ is equivalent to $\Sigma(\omega)=\left(d, 0,0,0, \cdots, 0,0, \gamma_{1}, \gamma_{2}, \delta, \theta\right)$. If $d$ is odd, we take a homeomorphism $h_{2}=\mu_{1} \tau_{1}^{(d+1) / 2} \mu_{1}^{-1}\left(\mathscr{D}_{2}^{-1} \mathscr{D}_{1}\right)^{2 z_{2} d-\gamma_{1}} \tau_{1}^{2+\left(2 z_{2} d-\gamma_{1}\right)\left(\gamma_{1}+\gamma_{2}-1\right)} \mu_{1}$. Then $\Sigma\left(\omega h_{1} h_{2}\right)=$ $(1,0,0,0, \cdots, 0,0,0, \gamma, \delta, \theta)$ is equivalent to $\Sigma(\omega)$.

If $n$ is even and $d$ is even, we take a homeomorphism $h_{2}^{\prime}=\mu_{1} \tau_{1}^{d / 2} \mu_{1}^{-2}$. Then $\Sigma\left(\omega h_{1} h_{2}^{\prime}\right)=\left(0,2,0,0, \cdots, 0,0,-2 z_{2} d+\gamma_{1}, 2 z_{2} d+\gamma_{2}, \delta, \theta\right)$ is equivalent 
to $\Sigma(\omega)$. Furthermore if $\gamma$ is odd, we take a homeomorphism $h_{3}=$ $\mathscr{D}_{2} \mathscr{D}_{1}^{-1} \tau_{1} \mu_{1}^{-1}\left(\mathscr{D}_{2}^{-1} \mathscr{D}_{1}\right)^{2+2 z_{2}-\gamma_{1}} \tau_{1}^{-2+\left(2+2 z_{2} d-\gamma_{1}\right)(\gamma-1)} \mu_{1}$. Then $\Sigma\left(\omega h_{1} h_{2}^{\prime} h_{3}\right)=(1,0,0,0, \cdots$, $0,0,0, \gamma, \delta, \theta)$ is equivalent to $\Sigma(\omega)$.

Lastly we may consider the case where $n$ is even, $d$ is even and $\gamma$ is even. If $\gamma_{1}$ and $\gamma_{2}$ are even, g.c.d $\left\{d, \gamma_{1}, \gamma_{2}\right\}$ is even which is contradiction. Therefore $\gamma_{1}$ and $\gamma_{2}$ are odd. Let

$$
h_{3}^{\prime}=\mu_{1}^{-1}\left(\mathscr{D}_{2}^{-1} \mathscr{D}_{1}\right)^{\left(2 z_{2} d-\gamma_{1}+1\right) / 2} \tau_{1}^{d / 2+\left(2 z_{2} d-\gamma_{1}+1\right) / 2 \cdot(\gamma-1) / 2} \mu_{1} \text {. }
$$

Then $\Sigma\left(\omega h_{1} h_{2}^{\prime} h_{3}^{\prime}\right)=(2,0,0,0, \cdots, 0,0,1, \gamma-1, \delta, \theta)$ is equivalent to $\Sigma(\omega)$. This completes the proof.

By the same way as Lemmas 2.3 and 2.4 in $\S 2$, we have;

Lemma 3.3. Any element $\left(\alpha, 0, \cdots, 0,0, \varepsilon, \gamma-\varepsilon, \delta_{1}, \delta_{2}, \cdots, \delta_{l}, \theta_{1}, \theta_{2}, \cdots\right.$, $\left.\theta_{m}\right)$ with $\alpha=1$ and $\varepsilon=0$ or $\alpha=2 \varepsilon=1$, is equivalent to $(\alpha, 0, \cdots, 0,0, \varepsilon$, $\left.\gamma^{\prime}-\varepsilon, \delta_{1}^{\prime}, \delta_{2}^{\prime}, \cdots, \delta_{l}^{\prime}, \theta_{1}^{\prime}, \theta_{2}^{\prime}, \cdots, \theta_{m}^{\prime}\right)$, where $0 \leqq \delta_{1}^{\prime} \leqq \delta_{2}^{\prime} \leqq \cdots \leqq \delta_{l}^{\prime} \leqq n / 2$ and $1 \leqq \theta_{1}^{\prime} \leqq$ $\theta_{2}^{\prime} \leqq \cdots \leqq \theta_{m}^{\prime} \leqq n / 2$.

LemmA 3.4. If $\Sigma(\omega)=\left(\alpha_{1}, \beta_{1}, \cdots, \alpha_{g}, \beta_{g}, \gamma_{1}, \gamma_{2}, \delta_{1}, \delta_{2}, \cdots, \delta_{l}, \theta_{1}, \theta_{2}, \cdots\right.$, $\left.\theta_{m}\right) \quad$ and $\quad \Sigma\left(\omega^{\prime}\right)=\left(\alpha_{1}^{\prime}, \beta_{1}^{\prime}, \cdots, \alpha_{g}^{\prime}, \beta_{g}^{\prime}, \gamma_{1}^{\prime}, \gamma_{2}^{\prime}, \delta_{1}^{\prime}, \delta_{2}^{\prime}, \cdots, \delta_{l}^{\prime}, \theta_{1}^{\prime}, \theta_{2}^{\prime}, \cdots, \theta_{m}^{\prime}\right)$ are equivalent elements of $Z_{n}^{-}(2 g+2 ; l, m)$ satisfying the following conditions;

(1) $0 \leqq \delta_{1} \leqq \delta_{2} \leqq \cdots \leqq \delta_{l} \leqq n / 2$,

(2) $1 \leqq \theta_{1} \leqq \theta_{2} \leqq \cdots \leqq \theta_{m} \leqq n / 2$,

(3) $0 \leqq \delta_{1}^{\prime} \leqq \delta_{2}^{\prime} \leqq \cdots \leqq \delta_{l}^{\prime} \leqq n / 2$ and

(4) $1 \leqq \theta_{1}^{\prime} \leqq \theta_{2}^{\prime} \leqq \cdots \leqq \theta_{m}^{\prime} \leqq n / 2$, then we have $\delta_{j}=\delta_{j}^{\prime}(1 \leqq j \leqq l)$ and $\theta_{k}=\theta_{k}^{\prime}(1 \leqq k \leqq m)$.

By the same way as Theorem 2.1 in $\S 2$, we have the following;

THEOREM 3.1. ( $n$; odd) A complete set of the equivalence classes of $Z_{n}^{-}(2 g+2 ; l, m), g \geqq 1$, is given by

$$
\mathscr{Z}_{n}^{-}(2 g+1 ; l, m)=\left\{\begin{array}{l}
\left(1,0,0,0, \cdots, 0,0,0, \gamma, \delta_{1}, \delta_{2}, \cdots, \delta_{l}, \theta_{1}, \theta_{2}, \cdots, \theta_{m}\right) ; \\
0 \leqq \delta_{1} \leqq \delta_{2} \leqq \cdots \leqq \delta_{l}<\frac{n}{2}, \\
1 \leqq \theta_{1} \leqq \theta_{2} \leqq \cdots \leqq \theta_{m}<\frac{n}{2}, \\
2 \gamma+\delta_{1}+\delta_{2}+\cdots+\delta_{l}+\theta_{1}+\theta_{2}+\cdots+\theta_{m} \equiv 0(\bmod n)
\end{array}\right\} .
$$

Now, we assume that $n$ is even. Suppose that an element $(\alpha, 0,0, \cdots$, $0,0, \varepsilon, \gamma-\varepsilon, \delta_{1}, \delta_{2}, \cdots, \delta_{l}, \theta_{1}, \theta_{2}, \cdots, \theta_{m}$ ) satisfies the conditions of Lemma 3.3. Then, by the same way as Lemmas 2.5 and 2.6, we have the 
following;

LEMMA 3.5. ( $n$; even) (1) If $n / 2 \leqq \gamma<n$ and $\delta_{l}=n / 2$ (or $\left.\theta_{m}=n / 2\right)$, then we have

$$
\begin{aligned}
\left(1,0, \cdots, 0,0,0, \gamma, \delta_{1}, \delta_{2}, \cdots \delta_{l}, \theta_{1}, \theta_{2}, \cdots, \theta_{m}\right) \\
\qquad\left(1,0, \cdots, 0,0,0, \gamma-\frac{n}{2}, \delta_{1}, \delta_{2}, \cdots, \delta_{l}, \theta_{1}, \theta_{2}, \cdots, \theta_{m}\right) .
\end{aligned}
$$

(2) If $n / 2 \leqq \gamma-1<n$ and $\delta_{l}=n / 2$ (or $\left.\theta_{m}=n / 2\right)$, then we have

$$
\begin{aligned}
\left(2,0, \cdots, 0,0,1, \gamma-1, \delta_{1}, \delta_{2}, \cdots, \delta_{l}, \theta_{1}, \theta_{2}, \cdots, \theta_{m}\right) \\
\quad \sim\left(2,0, \cdots, 0,0,1, \gamma-1-\frac{n}{2}, \delta_{1}, \delta_{2}, \cdots, \delta_{l}, \theta_{1}, \theta_{2}, \cdots, \theta_{m}\right) .
\end{aligned}
$$

LEMMA 3.6. Suppose that $\Sigma(\omega)=\left(\alpha_{1}, \beta_{1}, \cdots, \alpha_{g}, \beta_{g}, \gamma_{1}, \gamma_{2}, \delta_{1}, \delta_{2}, \cdots, \delta_{l}\right.$, $\left.\theta_{1}, \theta_{2}, \cdots, \theta_{m}\right)$ and $\Sigma\left(\omega^{\prime}\right)=\left(\alpha_{1}^{\prime}, \beta_{1}^{\prime}, \cdots, \alpha_{g}^{\prime}, \beta_{g}^{\prime}, \gamma_{1}^{\prime}, \gamma_{2}^{\prime}, \delta_{1}^{\prime}, \delta_{2}^{\prime}, \cdots, \delta_{l}^{\prime}, \theta_{1}^{\prime}, \theta_{2}^{\prime}, \cdots, \theta_{m}^{\prime}\right)$ are equivalent elements of $Z_{n}^{-}(2 g+2 ; l, m)$. If all numbers $\alpha_{i}, \beta_{i}, \delta_{j}, \theta_{k}$ are even, then all numbers $\alpha_{i}^{\prime}, \beta_{i}^{\prime}, \delta_{j}^{\prime}, \theta_{k}^{\prime}$ also are even.

THEOREM 3.2. ( $n$; even) A complete set of the equivalence classes of $Z_{n}^{-}(2 g+2 ; l, m), g \geqq 1$, is given by disjoint union $\mathscr{Y}_{n}^{-}(2 g+2 ; l, m)$ of the following sets;

$$
\begin{aligned}
& \mathscr{\varkappa}_{n}^{-}(2 g+2 ; l, m)_{1}^{0}=\left\{\begin{array}{l}
\left(1,0,0,0, \cdots, 0,0,0, \gamma, \delta_{1}, \delta_{2}, \cdots, \delta_{l}, \theta_{1}, \theta_{2}, \cdots, \theta_{m}\right) ; \\
0 \leqq \delta_{1} \leqq \delta_{2} \leqq \cdots \leqq \delta_{l}<\frac{n}{2}, \\
1 \leqq \theta_{1} \leqq \theta_{2} \leqq \cdots \leqq \theta_{m}<\frac{n}{2}, \\
2 \gamma+\delta_{1}+\delta_{2}+\cdots+\delta_{l}+\theta_{1}+\theta_{2}+\cdots+\theta_{m} \equiv 0(\bmod n)
\end{array}\right\} \\
& \mathscr{Z}_{n}^{-}(2 g+2 ; l, m)_{1}^{*}=\left\{\begin{array}{l}
\left(1,0,0,0, \cdots, 0,0,0, \gamma, \delta_{1}, \delta_{2}, \cdots, \delta_{l}, \theta_{1}, \theta_{2}, \cdots, \theta_{m}\right) ; \\
\delta_{l}=\frac{n}{2} \text { or } \theta_{m}=\frac{n}{2}, 0 \leqq \gamma<\frac{n}{2}, \\
0 \leqq \delta_{1} \leqq \delta_{2} \leqq \cdots \leqq \delta_{l} \leqq \frac{n}{2}, \\
1 \leqq \theta_{1} \leqq \theta_{2} \leqq \cdots \leqq \theta_{m} \leqq \frac{n}{2}, \\
2 \gamma+\delta_{1}+\delta_{2}+\cdots+\delta_{l}+\theta_{1}+\theta_{2}+\cdots+\theta_{m} \equiv 0(\bmod n)
\end{array}\right\}
\end{aligned}
$$




$$
\begin{aligned}
& \mathscr{Z}_{n}^{-}(2 g+2 ; l, m)_{2}^{0}=\left\{\begin{array}{l}
\left(2,0,0,0, \cdots, 0,0,1, \gamma, \delta_{1}, \delta_{2}, \cdots, \delta_{l}, \theta_{1}, \theta_{2}, \cdots, \theta_{m}\right) ; \\
\gamma ; \text { odd }, \delta_{j}, \theta_{k} ; \text { even }, \\
0 \leqq \delta_{1} \leqq \delta_{2} \leqq \cdots \leqq \delta_{l}<\frac{n}{2}, \\
1 \leqq \theta_{1} \leqq \theta_{2} \leqq \cdots \leqq \theta_{m}<\frac{n}{2}, \\
2+2 \gamma+\delta_{1}+\delta_{2}+\cdots+\delta_{l}+\theta_{1}+\theta_{2}+\cdots+\theta_{m} \equiv 0 \quad(\bmod n)
\end{array}\right) \\
& \mathscr{Z}_{n}^{-}(2 g+2 ; l, m)_{2}^{*}=\left\{\begin{array}{c}
\left(2,0,0,0, \cdots, 0,0,1, \gamma, \delta_{1}, \delta_{2}, \cdots, \delta_{l}, \theta_{1}, \theta_{2}, \cdots, \theta_{m}\right) ; \\
\delta_{l}=\frac{n}{2} \quad o r \quad \theta_{m}=\frac{n}{2}, 0 \leqq \gamma<\frac{n}{2}, \\
\gamma ; \text { odd }, \quad \delta_{j}, \theta_{k} ; e v e n, \\
0 \leqq \delta_{1} \leqq \delta_{2} \leqq \cdots \leqq \delta_{l} \leqq \frac{n}{2}, \\
1 \leqq \theta_{1} \leqq \theta_{2} \leqq \cdots \leqq \theta_{m} \leqq \frac{n}{2}, \\
2+2 \gamma+\delta_{1}+\delta_{2}+\cdots+\delta_{l}+\theta_{1}+\theta_{2}+\cdots+\theta_{m} \equiv 0(\bmod n)
\end{array}\right)
\end{aligned}
$$

Proof. By Lemmas 3.2, 3.3 and 3.5 , any element of $Z_{n}^{-}(2 g+2 ; l, m)$ is equivalent to an element of $\mathscr{Z}_{n}^{-}(2 g+2 ; l, m)$. Hence it is sufficient to prove that two distinct elements of $\mathscr{Z}_{n}^{-}(2 g+2 ; l, m)$ are not equivalent.

We have this theorem in a similar way to Theorem 2.2 except for the case of (iv) which will be obtained by a little modification. So we will prove it in the case of (iv).

(iv) Let $\Sigma(\omega)=\left(\alpha, 0,0,0, \cdots, 0,0, \varepsilon, \gamma, \delta_{1}, \delta_{2}, \cdots, \delta_{l}, \theta_{1}, \theta_{2}, \cdots, \theta_{m}\right)$ and $\Sigma\left(\omega^{\prime}\right)=\left(\alpha, 0,0,0, \cdots, 0,0, \varepsilon, \gamma^{\prime}, \delta_{1}^{\prime}, \delta_{2}^{\prime}, \cdots, \delta_{l}^{\prime}, \theta_{1}^{\prime}, \theta_{2}^{\prime}, \cdots, \theta_{m}^{\prime}\right)$ be equivalent elements of $\mathscr{\varkappa}_{n}^{-}(2 g+2 ; l, m)_{\alpha}^{0}$, where $\alpha=1$ and $\varepsilon=0$ or $\alpha=2$ and $\varepsilon=1$. Then, there exists a homeomorphism $h$ of $(X, S)$ onto itself which is a composition of elements in $\left\{\rho^{ \pm}, \rho_{1 i}, \tau_{1}^{ \pm}, \mu_{1}^{ \pm}, \theta_{12}^{ \pm}, \partial_{j}, \sigma_{k}, \partial_{a}^{ \pm}, \sigma_{a}^{ \pm}, \partial_{\gamma_{1}}, \partial_{\gamma_{2}}, \mathscr{Y}_{2}, \mathscr{D}_{1}^{ \pm}\right.$, $\left.\mathscr{D}_{2}^{ \pm}\right\}$such that $\omega^{\prime}=\omega h_{*}$. By Lemma 3.4, we have $\delta_{j}=\delta_{j}^{\prime}(1 \leqq j \leqq l)$ and $\theta_{k}=\theta_{k}^{\prime}(1 \leqq k \leqq m)$. Now, it is sufficient to prove the following Lemma;

LEMMA 3.7. $\gamma=\gamma^{\prime}$.

Proof. By Proposition 3.3, we may assume $h=g g^{\prime}$ where $g^{\prime}$ is an automorphism induced by a composition of homeomorphisms in $\left\{\partial_{j}, \sigma_{k}\right\}$ and $g$ is an automorphism induced by a composition of homeomorphisms in $\left\{\rho^{ \pm}, \rho_{1 i}, \tau_{1}^{ \pm}, \mu_{1}^{ \pm}, \theta_{12}^{ \pm}, \partial_{a, j}^{ \pm}, \partial_{1, j}, \partial_{2, j}, \sigma_{a, k}^{ \pm}, \sigma_{1, k}, \sigma_{2, k}, \mathscr{Y}_{2}, \mathscr{D}_{1}^{ \pm}, \mathscr{D}_{2}^{ \pm}\right\}$. Since $\partial_{1, j}=$ $\mathscr{D}_{2}^{-1} \mathscr{Y}_{2} \partial_{2, j} \mathscr{Y}_{2} \mathscr{D}_{2} \partial_{a, j}^{2}$ and $\sigma_{1, k}=\mathscr{D}_{2}^{-1} \mathscr{Y}_{2} \sigma_{2, k} \mathscr{Y}_{2} \mathscr{D}_{2} \sigma_{a, k}^{2}$, we may assume that $g$ is an automorphism induced by a composition of homeomorphisms in 
$\left\{\rho^{ \pm}, \rho_{1 i}, \tau_{1}^{ \pm}, \mu_{1}^{ \pm}, \theta_{12}^{ \pm}, \partial_{a, j}^{ \pm}, \partial_{2, j}, \sigma_{a, k}^{ \pm}, \sigma_{2, k}, \mathscr{V}_{2}, \mathscr{D}_{1}^{ \pm}, \mathscr{D}_{2}^{ \pm}\right\}$. We note that only an automorphism $\partial_{2, j}$ (resp. $\sigma_{2, k}$ ) can change $\gamma$. By the same way as Lemma 2.7, we complete the proof.

Using the homology group of a covering space of $X$ or by a geometric consideration, we have the following;

Proposition 3.4. (1) An element $(f, M)$ of $P_{n}$ corresponding to an element of $\mathscr{Z}_{n}^{-}(2 g+2 ; l, m)$ belongs to $P_{n}^{0}$, if $n$ is odd.

(2) An element $(f, M)$ of $P_{n}$ corresponding to an element of $\mathscr{\varkappa}_{n}^{-}(2 g+2 ; l, m)_{1}^{0}$ or $\mathscr{Z}_{n}^{-}(2 g+2 ; l, m)_{1}^{*}$ belongs to $P_{n}^{0}$, if $n$ is even.

(3) An element $(f, M)$ of $P_{n}$ corresponding to an element of $\mathscr{Z}_{n}^{-}(2 g+2 ; l, m)_{2}^{0}$ or $\mathscr{Z}_{n}^{-}(2 g+2 ; l, m)_{2}^{*}$ belongs to $P_{n}^{-}$, if $n$ is even.

§ 4. Determination of the equivalence classes of $P_{n}\left(X_{2}, S\right)$.

In this section, we will determine the equivalence classes of $P_{n}\left(X_{2}, S\right)$ which have been excluded in $\S 3$.

We have the following;

Proposition 4.1. The homeotopy group of $(X, S)$ is generated by $\mathscr{Y}_{2}, \mathscr{D}_{2}, \partial_{j}, \sigma_{k}, \partial_{r_{1}}, \partial_{r_{2}}, \sigma_{r_{1}}$ and $\sigma_{r_{2}}$, where $X=X_{2}$.

Among the automorphisms induced by them, we have easily the following equations;

PROPOSITION 4.2. (1) $\partial_{j} \mathscr{Y}_{2}=\mathscr{Y}_{2} \partial_{j}, \partial_{j} \mathscr{D}_{2}^{ \pm}=\mathscr{D}_{2}^{ \pm} \partial_{j}, \sigma_{k} \mathscr{Y}_{2}=\mathscr{Y}_{2} \sigma_{k}, \sigma_{k} \mathscr{D}_{2}^{ \pm}=$ $\mathscr{D}_{2}^{ \pm} \sigma_{k}$

(2) $\partial_{j} \sigma_{k}=\sigma_{k} \partial_{j}, \partial_{j} \sigma_{1, k}=\sigma_{1, k} \partial_{j}, \partial_{j} \sigma_{2, k}=\sigma_{2, k} \partial_{j}, \sigma_{k} \partial_{1, j}=\partial_{1, j} \sigma_{k}, \sigma_{k} \partial_{1, j}=\partial_{1, j} \sigma_{k}$,

(3) $\partial_{i} \partial_{1, j}=\partial_{1, j} \partial_{i}, \partial_{i} \partial_{2, j}=\partial_{2, j} \partial_{i},(i \neq j)$,

(4) $\partial_{i} \partial_{j} \partial_{1, j}=\partial_{1, i} \partial_{i} \partial_{j}, \partial_{i} \partial_{j} \partial_{2, j}=\partial_{2, i} \partial_{i} \partial_{j}, \quad(i \neq j)$,

(5) $\sigma_{i} \sigma_{1, k}=\sigma_{1, k} \sigma_{i}, \sigma_{i} \sigma_{2, k}=\sigma_{2, k} \sigma_{i},(i \neq k)$,

(6) $\sigma_{i} \sigma_{k} \sigma_{1, k}=\sigma_{1, i} \sigma_{i} \sigma_{k}, \sigma_{i} \sigma_{k} \sigma_{2, k}=\sigma_{2, i} \sigma_{i} \sigma_{k}(i \neq k)$,

(7) $\quad \mathscr{Y}_{2} \partial_{1, j}=\partial_{1, j} \mathscr{Y}_{2}, \quad \mathscr{Y}_{2} \partial_{2, j}=\partial_{1, j} \partial_{2, j} \partial_{1, j} \mathscr{Y}_{2}, \quad \mathscr{D}_{2} \partial_{1, j}=\partial_{1, j} \partial_{2, j} \partial_{1, j} \mathscr{D}_{2}, \quad \mathscr{D}_{2} \partial_{2, j}=$ $\partial_{1, j} \mathscr{D}_{2}, \mathscr{D}_{2}^{-1} \partial_{1, j}=\partial_{2, j} \mathscr{D}_{2}^{-1}, \mathscr{D}_{2}^{-1} \partial_{2, j}=\partial_{2, j} \partial_{1, j} \partial_{2, j} \mathscr{D}_{2}^{-1}$,

(8) $\mathscr{Y}_{2} \sigma_{1, k}=\sigma_{1, k} \mathscr{Y}_{2}, \mathscr{Y}_{2} \sigma_{2, k}=\sigma_{1, k} \sigma_{2, k} \sigma_{1, k} \mathscr{Y}_{2}, \mathscr{D}_{2} \sigma_{1, k}=\sigma_{1, k} \sigma_{2, k} \sigma_{1, k} \mathscr{D}_{2}, \mathscr{D}_{,} \sigma_{2, k}=$ $\sigma_{1, k} \mathscr{D}_{2}, \mathscr{D}_{2}^{-1} \sigma_{1, k}=\sigma_{2, k} \mathscr{D}_{2}^{-1}, \mathscr{D}_{2}^{-1} \sigma_{2, k}=\sigma_{2, k} \sigma_{1, k} \sigma_{2, k} \mathscr{D}_{2}^{-1}$,

(9) $\partial_{1, i} \partial_{1, j}=\partial_{1, j} \partial_{1, i}, \partial_{1, i} \partial_{2, j}=\partial_{2, j} \partial_{1, j},(i \neq j), \quad\left(\partial_{2, j} \partial_{1, j}\right)=\left(\partial_{1, j} \partial_{2, j}\right)^{-1}, \sigma_{1, i} \sigma_{1, k}=$ $\sigma_{1, k} \sigma_{1, i}, \sigma_{1, i} \sigma_{2, k}=\sigma_{2, k} \sigma_{1, i},(i \neq k), \sigma_{2, k} \sigma_{1, k}=\left(\sigma_{1, k} \sigma_{2, k}\right)^{-1}$,

(10) $\mathscr{D}_{2}^{-1} \mathscr{Y}_{2}=\mathscr{Y}_{2} \mathscr{D}_{2},\left(\mathscr{Y}_{2}{ }^{-1}=\mathscr{Y}_{2}\right)$, where $\partial_{1, j}=\partial_{j} \partial_{\gamma_{1}} \partial_{j}, \partial_{2, j}=\partial_{j} \partial_{r_{2}} \partial_{j}, \sigma_{1, k}=\sigma_{k} \sigma_{r_{1}} \sigma_{k}$ and $\sigma_{2, k}=\sigma_{k} \sigma_{\gamma_{2}} \sigma_{k}$.

Proposition 4.3. If $\left(\gamma_{1}, \gamma_{2}, \delta_{1}, \delta_{2}, \cdots, \delta_{l}, \theta_{1}, \theta_{2}, \cdots, \theta_{m}\right)$ and $\left(\gamma_{1}^{\prime}, \gamma_{2}^{\prime}, \delta_{1}^{\prime}\right.$, 
$\left.\delta_{2}^{\prime}, \cdots, \delta_{l}^{\prime}, \theta_{1}^{\prime}, \theta_{2}^{\prime}, \cdots, \theta_{m}^{\prime}\right)$ are equivalent elements of $Z_{n}^{-}(2 ; l, m)$, then we have $d=d^{\prime}$ and $\alpha=\alpha^{\prime}$, where $d=$ g.c.d. $\left\{\delta_{1}, \delta_{2}, \cdots, \delta_{l}, \theta_{1}, \theta_{2}, \cdots, \theta_{m}, n\right\}$, $\alpha=$ g.c.d. $\left\{\gamma_{1}+\gamma_{2}, d\right\}, \quad d^{\prime}=$ g.c.d. $\left\{\delta_{1}^{\prime}, \delta_{2}^{\prime}, \cdots, \delta_{l}^{\prime}, \theta_{1}^{\prime}, \theta_{2}^{\prime}, \cdots, \theta_{m}^{\prime}, n\right\}, \quad$ and $\quad \alpha^{\prime}=$ g.c.d. $\left\{\gamma_{1}^{\prime}+\gamma_{2}^{\prime}, d^{\prime}\right\}$.

Lemma 4.1. Any element $\left(\gamma_{1}, \gamma_{2}, \delta_{1}, \delta_{2}, \cdots, \delta_{l}, \theta_{1}, \theta_{2}, \cdots, \theta_{m}\right)$ is equivalent to an element $\left(\gamma_{1}^{\prime}, \gamma_{2}^{\prime}, \delta_{1}^{\prime}, \delta_{2}^{\prime}, \cdots, \delta_{l}^{\prime}, \theta_{1}^{\prime}, \theta_{2}^{\prime}, \cdots, \theta_{m}^{\prime}\right)$, where $0 \leqq \delta_{1}^{\prime} \leqq \delta_{2}^{\prime} \leqq \cdots$ $\leqq \delta_{l}^{\prime} \leqq n / 2$ and $1 \leqq \theta_{1}^{\prime} \leqq \theta_{2}^{\prime} \leqq \cdots \leqq \theta_{m}^{\prime} \leqq n / 2$.

For any element $\Sigma(\omega)=\left(\gamma_{1}, \gamma_{2}, \delta, \theta\right)$ of $Z_{n}^{-}(2 ; l, m)$, applying $h=\mathscr{D}_{2}^{-1} \mathscr{Y}_{2}$, we have $\Sigma(\omega h)=\left(\gamma_{2}, \gamma_{1}, \delta, \theta\right)$. Hence we have;

Lemma 4.2. $\left(\gamma_{1}, \gamma_{2}, \delta, \theta\right) \sim\left(\gamma_{2}, \gamma_{1}, \delta, \theta\right)$.

LEMMA 4.3. If $\gamma_{1}+\gamma_{2}>n$, then we have $\left(\gamma_{1}, \gamma_{2}, \delta, \theta\right) \sim\left(\gamma_{1}^{\prime}, \gamma_{2}^{\prime}, \delta, \theta\right)$ where $n>\gamma_{1}^{\prime}+\gamma_{2}^{\prime}>0$.

Proof. There exists a positive integer $x$ such that $x\left(\gamma_{1}+\gamma_{2}-n\right) \geqq \gamma_{2}$. We take a positive integer $x_{0}=\min \left\{x \in N ; x(\gamma-n) \geqq \gamma_{2}\right\}$, where $\gamma=\gamma_{1}+\gamma_{2}$. Let $\gamma_{1}^{\prime}=x_{0} \gamma_{1}+\left(x_{0}-1\right) \gamma_{2}-x_{0} n$ and $\gamma_{2}^{\prime}=\left(x_{0}-1\right) n-\left(x_{0}-1\right) \gamma_{1}-\left(x_{0}-2\right) \gamma_{2}$. Because of the choice of $x_{0}$, we have the following;

(i) $n>\gamma_{1}^{\prime} \geqq 0$ and $\gamma_{1}^{\prime} \equiv x_{0} \gamma_{1}+\left(x_{0}-1\right) \gamma_{2}(\bmod n)$,

(ii) $n>\gamma_{2}^{\prime} \geqq 0$ and $\gamma_{2}^{\prime} \equiv-\left(x_{0}-1\right) \gamma_{1}-\left(x_{0}-2\right) \gamma_{2}(\bmod n)$.

Hence we take a homeomorphism $h=\mathscr{D}_{2}^{1-x_{0}}$. Then $\Sigma(\omega h)=\left(\gamma_{1}^{\prime}, \gamma_{2}^{\prime}, \delta, \theta\right)$ is equivalent to $\Sigma(\omega)$, which completes the proof.

LEMMA 4.4. If an element $\left(\gamma_{1}, \gamma_{2}, \delta, \theta\right)$ of $Z_{n}^{-}(2 ; l, m)$ satisfies the conditions $0 \leqq \gamma_{1} \leqq \gamma_{2}<n$ and $0 \leqq \gamma_{1}+\gamma_{2} \leqq n$, then there exists an element $\left(\gamma_{1}^{\prime}, \gamma_{2}^{\prime}, \delta^{\prime}, \theta^{\prime}\right)$ which is equivalent to $\left(\gamma_{1}, \gamma_{2}, \delta, \theta\right)$ such that $\gamma_{1}^{\prime}$ and $\gamma_{2}^{\prime}$ satisfy the conditions; $0 \leqq \gamma_{1}^{\prime} \leqq \gamma_{2}^{\prime}<n, 0 \leqq \gamma_{1}^{\prime}+\gamma_{2}^{\prime} \leqq n$ and $0 \leqq \gamma_{1}^{\prime} \leqq[\alpha / 2]$, where [x] is the largest integer $\leqq x$ and $\alpha$ is an integer in Proposition 4.3.

Proof. Since $\alpha=$ g.c.d. $\left\{\gamma_{1}+\gamma_{2}, \delta_{1}, \delta_{2}, \cdots, \delta_{l}, \theta_{1}, \theta_{2}, \cdots, \theta_{m}, n\right\}$, there exist integers $z, x_{1}, x_{2}, \cdots, x_{l}, y_{1}, y_{2}, \cdots, y_{m}$ such that $z\left(\gamma_{1}+\gamma_{2}\right)+x_{1} \delta_{1}+$ $x_{2} \delta_{2}+\cdots+x_{l} \delta_{l}+y_{1} \theta_{1}+y_{2} \theta_{2}+\cdots+y_{m} \theta_{m} \equiv \alpha(\bmod n)$. We take a non-negative integer $i_{0}$ such that $0 \leqq \gamma_{1}-i_{0} \alpha<\alpha$. If $0 \leqq \gamma_{1}-i_{0} \alpha \leqq[\alpha / 2]$, we take a homeomorphism $h=\mathscr{D}_{2}^{-z}\left(\partial_{1,1} \partial_{2,1}\right)^{x_{1}}\left(\partial_{1,2} \partial_{2,2}\right)^{x_{2}} \ldots\left(\partial_{1, l} \partial_{2, l}\right)^{x_{l}}\left(\sigma_{1,1} \sigma_{2,1}\right)^{y_{1}}\left(\sigma_{1,2} \sigma_{2,2}\right)^{y_{2}} \ldots$ $\left(\sigma_{1, m} \sigma_{2, m}\right)^{\nu_{m}}$. Then $\Sigma\left(\omega h^{-i_{0}}\right)=\left(\gamma_{1}-i_{0} \alpha, \gamma_{2}+i_{0} \alpha, \delta, \theta\right)$ is equivalent to $\Sigma(\omega)$, and we have $0 \leqq \gamma_{1}-i_{0} \alpha \leqq \gamma_{2}+i_{0} \alpha<n$, which is satisfied the conditions of Lemma.

If $[\alpha / 2]<\gamma_{1}-i_{0} \alpha<\alpha$, then $\Sigma\left(\omega h^{i_{0}} \mathscr{D}_{2}^{-2} \mathscr{Y}_{2} h^{1-n / \alpha}\right)=\left(\gamma_{1}^{\prime}, \gamma_{2}^{\prime}, \delta, \theta\right)$ is equivalent to $\Sigma(\omega)$, where $\gamma_{1}^{\prime}=\alpha-\gamma_{1}+i_{0} \alpha$ and $\gamma_{2}^{\prime}=2 \gamma_{1}+\gamma_{2}-i_{0} \alpha-\alpha$. $\gamma_{1}^{\prime}$ and $\gamma_{2}^{\prime}$ satisfy the conditions $0 \leqq \gamma_{1}^{\prime} \leqq[\alpha / 2], 0 \leqq \gamma_{1}^{\prime} \leqq \gamma_{2}^{\prime}<n$ and $0 \leqq \gamma_{1}^{\prime}+\gamma_{2}^{\prime}<n$. 
By the same way as Lemma 2.4, we have;

LEMMA 4.5. If $\Sigma(\omega)=\left(\gamma_{1}, \gamma_{2}, \delta_{1}, \delta_{2}, \cdots, \delta_{l}, \theta_{1}, \theta_{2}, \cdots, \theta_{m}\right)$ and $\Sigma\left(\omega^{\prime}\right)=$ $\left(\gamma_{1}^{\prime}, \gamma_{2}^{\prime}, \delta_{1}^{\prime}, \delta_{2}^{\prime}, \cdots, \delta_{l}^{\prime}, \theta_{1}^{\prime}, \theta_{2}^{\prime}, \cdots, \theta_{m}^{\prime}\right)$ are equivalent elements of $Z_{n}^{-}(2 ; l, m)$ satisfying the following conditions; (1) $0 \leqq \delta_{1} \leqq \delta_{2} \leqq \cdots \leqq \delta_{l} \leqq n / 2$, (2) $1 \leqq$ $\theta_{1} \leqq \theta_{2} \leqq \cdots \leqq \theta_{m} \leqq n / 2$, (3) $0 \leqq \delta_{1}^{\prime} \leqq \delta_{2}^{\prime} \leqq \cdots \leqq \delta_{l}^{\prime} \leqq n / 2$ and (4) $1 \leqq \theta_{1}^{\prime} \leqq \theta_{2}^{\prime} \leqq \cdots \leqq$ $\theta_{m}^{\prime} \leqq n / 2$, then we have $\delta_{j}=\delta_{j}^{\prime}(1 \leqq j \leqq l)$ and $\theta_{k}=\theta_{k}^{\prime}(1 \leqq k \leqq m)$.

THEOREM 4.1. ( $n$; odd) A complete set of the equivalence classes of $Z_{n}^{-}(2 ; l, m)$ is given by;

$$
\mathscr{\varkappa}_{n}^{-}(2 ; l, m)=\left\{\begin{array}{l}
\left(\gamma_{1}, \gamma_{2}, \delta_{1}, \delta_{2}, \cdots, \delta_{l}, \theta_{1}, \theta_{2}, \cdots, \theta_{m}\right) ; \\
0 \leqq \delta_{1} \leqq \delta_{2} \leqq \cdots \leqq \delta_{l}<\frac{n}{2}, \\
1 \leqq \theta_{1} \leqq \theta_{2} \leqq \cdots \leqq \theta_{m}<\frac{n}{2}, \\
2 \gamma_{1}+2 \gamma_{2}+\delta_{1}+\delta_{2}+\cdots+\delta_{l}+\theta_{1}+\theta_{2}+\cdots+\theta_{m} \equiv 0 \quad(\bmod n) \\
0 \leqq \gamma_{1} \leqq \gamma_{2}<n, \quad 0 \leqq \gamma_{1} \leqq\left[\frac{\alpha}{2}\right], \quad \gamma_{1}+\gamma_{2} \leqq n, \\
\text { g.c.d. }\left\{\gamma_{1}, \gamma_{2}, \delta_{1}, \delta_{2}, \cdots, \delta_{l}, \theta_{1}, \theta_{2}, \cdots, \theta_{m}, n\right\}=1
\end{array}\right\}
$$

where $\alpha=$ g.c.d. $\left\{\gamma_{1}+\gamma_{2}, \delta_{1}, \delta_{2}, \cdots, \delta_{l}, \theta_{1}, \theta_{2}, \cdots, \theta_{m}, n\right\}$.

Proof. By Lemmas 4.1, 4.2, 4.3 and 4.4, any element $\left(\gamma_{1}, \gamma_{2}, \delta, \theta\right)$ of $Z_{n}^{-}(2 ; l, m)$ is equivalent to an element of $\mathscr{Z}_{n}^{-}(2 ; l, m)$. Hence it is sufficient to prove that two distinct elements of $\mathscr{\varkappa}_{n}^{-}(2 ; l, m)$ are not equivalent.

Let $\Sigma(\omega)=\left(\gamma_{1}, \gamma_{2}, \delta_{1}, \delta_{2}, \cdots, \delta_{l}, \theta_{1}, \theta_{2}, \cdots, \theta_{m}\right)$ and $\Sigma\left(\omega^{\prime}\right)=\left(\gamma_{1}^{\prime}, \gamma_{2}^{\prime}, \delta_{1}^{\prime}\right.$, $\left.\delta_{2}^{\prime}, \cdots, \delta_{l}^{\prime}, \theta_{1}^{\prime}, \theta_{2}^{\prime}, \cdots, \theta_{m}^{\prime}\right)$ be equivalent elements of $\mathscr{\gamma}_{n}^{-}(2 ; l, m)$. Then there exists a homeomorphism $h$ of $(X, S)$ onto itself which is a composition of elements in $\left\{\mathscr{Y}_{2}, \mathscr{D}_{2}^{ \pm}, \partial_{j}, \sigma_{k}, \partial_{r_{1}}, \partial_{r_{2}}, \sigma_{r_{1}}, \sigma_{\gamma_{2}}\right\}$. By Lemma 4.5, we have $\delta_{j}=\delta_{j}^{\prime}(1 \leqq j \leqq l)$ and $\theta_{k}=\theta_{k}^{\prime}(1 \leqq k \leqq m)$. Now, it is sufficient to prove the following lemma;

LEMMA 4.6. $\gamma_{1}=\gamma_{1}^{\prime}$ and $\gamma_{2}=\gamma_{2}^{\prime}$.

Proof. By Proposition 4.2, we may assume that $h_{*}=h_{1} h_{2} h_{3} h_{4}$ where $h_{1}$ is an automorphism induced by a composition of homeomorphisms in $\left\{\partial_{1, j}, \partial_{2, j}, \sigma_{1, k}, \sigma_{2, k}\right\}, h_{2}=\mathscr{D}_{2}^{z}\left(z\right.$ is an integer), $h_{3}=\mathscr{Y}_{2}^{\varepsilon}(\varepsilon=1$ or 0$)$, and $h_{4}$ is an automorphism induced by a composition of homeomorphisms in $\left\{\partial_{j}, \sigma_{k}\right\}$. Moreover we may assume that $h_{1}=g_{1} g_{2} \cdots g_{l} g_{1}^{\prime} g_{2}^{\prime} \cdots g_{m}^{\prime}$ where $g_{j}=\left(\partial_{1, j} \partial_{2, j}\right)^{x_{j}}$ or $\partial_{2, j}\left(\partial_{1, j} \partial_{2, j}\right)^{x_{j}}\left(x_{j}\right.$ is an integer $)$ and $g_{k}^{\prime}=\left(\sigma_{1, k} \sigma_{2, k}\right)^{y_{k}}$ or $\sigma_{2, k}\left(\sigma_{1, k} \sigma_{2, k}\right)^{y_{k}}\left(y_{k}\right.$ is 
an integer). (If $\delta_{j}=0$, then $g_{j}$ is the identity.)

Since $h_{1}$ can not change $\delta_{j}$ and $\theta_{k}$, we may assume that $g_{j}=\left(\partial_{1, j} \partial_{2, j}\right)^{x_{j}}$ and $g_{k}^{\prime}=\left(\sigma_{1, k} \sigma_{2, k}\right)^{\nu_{k}}$. We note that only $h_{4}$ can change $\gamma_{1}$ or $\gamma_{2}$. So we have;

$$
\begin{aligned}
& \left\{\begin{aligned}
\gamma_{1}^{\prime}= & \gamma_{1}+\left(x_{1} \delta_{1}+x_{2} \delta_{2}+\cdots+x_{l} \delta_{l}+y_{1} \theta_{1}+y_{2} \theta_{2}+\cdots+y_{m} \theta_{m}\right)-z\left(\gamma_{1}+\gamma_{2}\right) \\
\gamma_{2}^{\prime}= & \gamma_{2}-\left(x_{1} \delta_{1}+x_{2} \delta_{2}+\cdots+x_{l} \delta_{l}+y_{1} \theta_{1}+y_{2} \theta_{2}+\cdots+y_{m} \theta_{m}\right)+z\left(\gamma_{1}+\gamma_{2}\right),
\end{aligned}\right. \\
& \quad \text { if } \varepsilon=0, \text { or } \\
& \left\{\begin{array}{l}
\gamma_{1}^{\prime}=\gamma_{2}-\left(x_{1} \delta_{1}+x_{2} \delta_{2}+\cdots+x_{l} \delta_{l}+y_{1} \theta_{1}+y_{2} \theta_{2}+\cdots+y_{m} \theta_{m}\right)+(z+1)\left(\gamma_{1}+\gamma_{2}\right) \\
\gamma_{2}^{\prime}=\gamma_{1}+\left(x_{1} \delta_{1}+x_{2} \delta_{2}+\cdots+x_{l} \delta_{l}+y_{1} \theta_{1}+y_{2} \theta_{2}+\cdots+y_{m} \theta_{m}\right)-(z+1)\left(\gamma_{1}+\gamma_{2}\right),
\end{array}\right. \\
& \quad \text { if } \varepsilon=1 .
\end{aligned}
$$

We will prove Lemma 4.6 in the case of (i) or (ii) respectively.

In the case of $(i)$, we have $\gamma_{1}^{\prime} \equiv \gamma_{1}(\bmod \alpha)$ and $\gamma_{2}^{\prime} \equiv \gamma_{2}(\bmod \alpha)$. Hence we have $\gamma_{1}^{\prime}=\gamma_{1}$ and $\gamma_{2}^{\prime}=\gamma_{2}$ since $0 \leqq \gamma_{1} \leqq[\alpha / 2]=(\alpha-1) / 2, \quad 0 \leqq \gamma_{1}^{\prime} \leqq[\alpha / 2]=$ $(\alpha-1) / 2$ and $\gamma_{1}^{\prime}+\gamma_{2}^{\prime}=\gamma_{1}+\gamma_{2}$.

In the case of $(\mathrm{ii})$, we have $\gamma_{1}^{\prime} \equiv \gamma_{2}(\bmod \alpha)$ and $\gamma_{2}^{\prime} \equiv \gamma_{1}(\bmod \alpha)$. Hence we have $\gamma_{1}+\gamma_{1}^{\prime} \equiv \gamma_{1}+\gamma_{2} \equiv 0(\bmod \alpha)$. Then, by elementary algebra, we have $\gamma_{1}=\gamma_{1}^{\prime}=0$ and $\gamma_{2}=\gamma_{2}^{\prime}$.

Now, we assume that $n$ is even. Suppose that an element $\left(\gamma_{1}, \gamma_{2}\right.$, $\left.\delta_{1}, \delta_{2}, \cdots, \delta_{l}, \theta_{1}, \theta_{2}, \cdots, \theta_{m}\right)$ satisfying the following conditions; (1) $0 \leqq \delta_{1} \leqq$ $\delta_{2} \leqq \cdots \leqq \delta_{l} \leqq n / 2$, (2) $1 \leqq \theta_{1} \leqq \theta_{2} \leqq \cdots \leqq \theta_{m} \leqq n / 2$, (3) $0 \leqq \gamma_{1} \leqq \gamma_{2}<n$, (4) $0 \leqq \gamma_{1}+$ $\gamma_{2} \leqq n$ and (5) $0 \leqq \gamma_{1} \leqq[\alpha / 2]$. Then, we have the following;

LEMMA 4.7. ( $n$; even) If $n / 2 \leqq \gamma_{2}$ or $n / 2<\gamma_{1}+\gamma_{2} \leqq n$, and $\delta_{l}=n / 2$ (or $\left.\theta_{m}=n / 2\right)$, then $\left(\gamma_{1}, \gamma_{2}, \delta_{1}, \delta_{2}, \cdots, \delta_{l}, \theta_{1}, \theta_{2}, \cdots, \theta_{m}\right)$ is equivalent to $\left(\gamma_{1}^{\prime}, \gamma_{2}^{\prime}, \delta_{1}\right.$, $\left.\delta_{2}, \cdots, \delta_{l}, \theta_{1}, \theta_{2}, \cdots, \theta_{m}\right)$ satisfying the following conditions; $0 \leqq \gamma_{1}^{\prime}+\gamma_{2}^{\prime} \leqq$ $n / 2,0 \leqq \gamma_{1}^{\prime} \leqq \gamma_{2}^{\prime}<n / 2$ and $0 \leqq \gamma_{1}^{\prime} \leqq[\alpha / 2]$.

Proof. If $n / 2 \leqq \gamma_{2}$, we apply $\partial_{2, l}$ (or $\sigma_{2, m}$ ). Then we have $\left(\gamma_{1}^{\prime}, \gamma_{2}^{\prime}, \delta_{1}\right.$, $\left.\delta_{2}, \cdots, \delta_{l}, \theta_{1}, \theta_{2}, \cdots, \theta_{m}\right) \sim\left(\gamma_{1}, \gamma_{2}, \delta_{1}, \delta_{2}, \cdots, \delta_{l}, \theta_{1}, \theta_{2}, \cdots, \theta_{m}\right)$ where $\gamma_{1}^{\prime}=\gamma_{1}$ and $\gamma_{2}^{\prime}=\gamma_{2}-n / 2$. Applying Lemma 4.2 if necessary, we may assume that $0 \leqq \gamma_{1}^{\prime} \leqq \gamma_{2}^{\prime}$. Hence we have $0 \leqq \gamma_{1}^{\prime}+\gamma_{2}^{\prime} \leqq n / 2,0 \leqq \gamma_{1}^{\prime} \leqq \gamma_{2}^{\prime}<n / 2$ and $0 \leqq \gamma_{1}^{\prime} \leqq[\alpha / 2]$.

If $0 \leqq \gamma_{2}<n / 2$ and $n / 2<\gamma_{1}+\gamma_{2}$, we apply $\partial_{2, l}$ (or $\sigma_{2, m}$ ). Then we have $\left(\gamma_{1}^{\prime}, \gamma_{2}^{\prime}, \delta, \theta\right) \sim\left(\gamma_{1}, \gamma_{2}, \delta, \theta\right)$, where $\gamma_{1}^{\prime}=\gamma_{1}$ and $\gamma_{2}^{\prime}=\gamma_{2}+n / 2$. Since $\gamma_{1}^{\prime}+\gamma_{2}^{\prime}>n$, by the same way as Lemma 4.3, $\left(\gamma_{1}^{\prime \prime}, \gamma_{2}^{\prime \prime}, \delta, \theta\right) \sim\left(\gamma_{1}^{\prime}, \gamma_{2}^{\prime}, \delta, \theta\right)$, where $\gamma_{1}^{\prime \prime}$ and $\gamma_{2}^{\prime \prime}$ satisfy the condition $\gamma_{1}^{\prime \prime}+\gamma_{2}^{\prime \prime}=\gamma_{1}^{\prime}+\gamma_{2}^{\prime}-n$. Hence we have $0<\gamma_{1}^{\prime \prime}+\gamma_{2}^{\prime \prime}<$ $n / 2$. Applying Lemmas 4.2 and 4.4 if necessary, we may assume that $0 \leqq \gamma_{1}^{\prime \prime} \leqq \gamma_{2}^{\prime \prime}$ and $\gamma_{1}^{\prime \prime} \leqq[\alpha / 2]$. Hence we have $0<\gamma_{1}^{\prime \prime}+\gamma_{2}^{\prime \prime}<n / 2,0 \leqq \gamma_{1}^{\prime \prime} \leqq \gamma_{2}^{\prime \prime}<n / 2$ and $0 \leqq \gamma_{1}^{\prime \prime} \leqq[\alpha / 2]$. 
THEOREM 4.2. ( $n$; even) A complete set of the equivalence classes of $Z_{n}^{-}(2 ; l, m)$ is given by the disjoint union $\mathscr{Y}_{n}^{-}(2 ; l, m)$ of the following sets;

$$
\begin{aligned}
& \mathscr{c}_{n}-(2 ; l, m)^{0}=\left\{\begin{array}{l}
\left(\gamma_{1}, \gamma_{2}, \delta_{1}, \delta_{2}, \cdots, \delta_{l}, \theta_{1}, \theta_{2}, \cdots, \theta_{m}\right) ; \\
0 \leqq \gamma_{1} \leqq \gamma_{2}<n, \gamma_{1}+\gamma_{2} \leqq n, \quad 0 \leqq \gamma_{1} \leqq\left[\frac{\alpha}{2}\right], \\
0 \leqq \delta_{1} \leqq \delta_{2} \leqq \cdots \leqq \delta_{l}<\frac{n}{2}, \\
1 \leqq \theta_{1} \leqq \theta_{2} \leqq \cdots \leqq \theta_{m}<\frac{n}{2}, \\
2 \gamma_{1}+2 \gamma_{2}+\delta_{1}+\delta_{2}+\cdots+\delta_{l}+\theta_{1}+\theta_{2}+\cdots+\theta_{m} \equiv 0 \quad(\bmod n), \\
\text { g.c.d. }\left\{\gamma_{1}, \gamma_{2}, d\right\}=1
\end{array}\right\} \\
& \mathscr{Z}_{n}-(2 ; l, m)^{*}=\left\{\begin{array}{l}
\left(\gamma_{1}, \gamma_{2}, \delta_{1}, \delta_{2}, \cdots, \delta_{l}, \theta_{1}, \theta_{2}, \cdots, \theta_{m}\right) ; \\
\delta_{l}=\frac{n}{2} \text { or } \theta_{m}=\frac{n}{2}, \quad 0 \leqq \gamma_{1} \leqq \gamma_{2}<\frac{n}{2}, \gamma_{1}+\gamma_{2} \leqq \frac{n}{2}, \\
0 \leqq \gamma_{1} \leqq\left[\frac{\alpha}{2}\right], \\
0 \leqq \delta_{1} \leqq \delta_{2} \leqq \cdots \leqq \delta_{l} \leqq \frac{n}{2}, \\
1 \leqq \theta_{1} \leqq \theta_{2} \leqq \cdots \leqq \theta_{m} \leqq \frac{n}{2}, \\
2 \gamma_{1}+2 \gamma_{2}+\delta_{1}+\delta_{2}+\cdots+\delta_{l}+\theta_{1}+\theta_{2}+\cdots+\theta_{m} \equiv 0 \quad(\bmod n) \\
\text { g.c.d. }\left\{\gamma_{1}, \gamma_{2}, d\right\}=1
\end{array}\right\}
\end{aligned}
$$

where $d=$ g.c.d. $\left\{\delta_{1}, \delta_{2}, \cdots, \delta_{l}, \theta_{1}, \theta_{2}, \cdots, \theta_{m}, n\right\}$ and $\alpha=$ g.c.d. $\left\{d, \gamma_{1}+\gamma_{2}\right\}$.

Proof. By Lemmas 4.1, 4.2, 4.3, 4.4 and 4.7, any element of $Z_{n}^{-}(2 ; l, m)$ is equivalent to an element of $\mathscr{Z}_{n}^{-}(2 ; l, m)$. Hence it is sufficient to prove that two distinct elements of $\mathscr{Z}_{n}^{-}(2 ; l, m)$ is not equivalent. We will prove it in respective cases.

(i) Let $\Sigma(\omega)$ be an element of $\mathscr{\varkappa}_{n}^{-}(2 ; l, m)^{0}$ and $\Sigma\left(\omega^{\prime}\right)$ be an element of $\mathscr{Z}_{n}^{-}(2 ; l, m)^{*}$. By Lemma 4.5 , it is impossible that $\Sigma(\omega)$ and $\Sigma\left(\omega^{\prime}\right)$ is equivalent.

(ii) Let $\Sigma(\omega)=\left(\gamma_{1}, \gamma_{2}, \delta_{1}, \delta_{2}, \cdots, \delta_{l}, \theta_{1}, \theta_{2}, \cdots, \theta_{m}\right)$ and $\Sigma\left(\omega^{\prime}\right)=\left(\gamma_{1}^{\prime}, \gamma_{2}^{\prime}\right.$, $\left.\delta_{1}^{\prime}, \delta_{2}^{\prime}, \cdots, \delta_{l}^{\prime}, \theta_{1}^{\prime}, \theta_{2}^{\prime}, \cdots, \theta_{m}^{\prime}\right)$ be equivalent elements of $\mathscr{\varkappa}_{n}^{-}(2 ; l, m)^{0}$. By a similar way as Theorem 4.1 and Lemma 4.6, we have $\Sigma(\omega)=\Sigma\left(\omega^{\prime}\right)$.

(iii) Let $\Sigma(\omega)=\left(\gamma_{1}, \gamma_{2}, \delta_{1}, \delta_{2}, \cdots, \delta_{l}, \theta_{1}, \theta_{2}, \cdots, \theta_{m}\right)$ and $\Sigma\left(\omega^{\prime}\right)=\left(\gamma_{1}^{\prime}, \gamma_{2}^{\prime}\right.$, $\left.\delta_{1}^{\prime}, \delta_{2}^{\prime}, \cdots, \delta_{l}^{\prime}, \theta_{1}^{\prime}, \theta_{2}^{\prime}, \cdots, \theta_{m}^{\prime}\right)$ be equivalent elements of $\mathscr{\varkappa}_{n}^{-}(2 ; l, m)^{*}$. Then there exists a homeomorphism $h$ of $(X, S)$ onto itself which is a com- 
position of elements on $\left\{\mathscr{Y}_{2}, \mathscr{D}_{2}^{ \pm}, \partial_{j}, \sigma_{k}, \partial_{\gamma_{1}}, \partial_{\gamma_{2}}, \sigma_{\gamma_{1}}, \sigma_{\gamma_{2}}\right\}$. By Lemma 4.5, we have $\delta_{j}=\delta_{j}^{\prime}(1 \leqq j \leqq l)$ and $\theta_{k}=\theta_{k}^{\prime}(1 \leqq k \leqq m)$. Now, it is sufficient to prove the following lemma;

LEMMA 4.8. $\gamma_{1}=\gamma_{1}^{\prime}$ and $\gamma_{2}=\gamma_{2}^{\prime}$.

By Proposition 4.2, we may assume that $h_{*}=h_{1} h_{2} h_{3} h_{4}$ where $h_{1}, h_{2}, h_{3}, h_{4}$ is the same automorphism as $h_{1}, h_{2}, h_{3}, h_{4}$ in Lemma 4.7 respectively. Moreover we may assume that $h_{1}=g_{1} g_{2} \cdots g_{l} g_{1}^{\prime} g_{2}^{\prime} \cdots g_{m}^{\prime}$ where $g_{j}=\left(\partial_{1, j} \partial_{2, j}\right)^{x_{j}}$ or $\partial_{2, j}\left(\partial_{1, j} \partial_{2, j}\right)^{x_{j}}\left(x_{j}\right.$ is an integer $)$ and $g_{k}^{\prime}=\left(\sigma_{1, k} \sigma_{2, k}\right)^{y_{k}}$ or $\sigma_{2, k}\left(\sigma_{1, k} \sigma_{2, k}\right)^{y_{k}}\left(y_{k}\right.$ is an integer) (If $\delta_{j}=0$ then $g_{j}$ is the identity.). Since $h_{1}$ can not change $\delta_{j}$ and $\theta_{k}$, we may assume that $g_{j}=\left(\partial_{1, j} \partial_{2, j}\right)^{x_{j}}$ if $\partial_{j}<n / 2$ and $g_{k}^{\prime}=\left(\sigma_{1, k} \sigma_{2, k}\right)^{y_{k}}$ if $\theta_{k}<n / 2$.

Let $\Sigma\left(\omega h_{1}\right)=\left(\gamma_{1}^{\prime \prime}, \gamma_{2}^{\prime \prime}, \delta, \theta\right)$. Then we have;

$$
\begin{aligned}
& \left\{\begin{array}{l}
\gamma_{1}^{\prime \prime} \equiv \gamma_{1}+\left(x_{1} \delta_{1}+x_{2} \delta_{2}+\cdots+x_{l} \delta_{l}+y_{1} \theta_{1}+y_{2} \theta_{2}+\cdots+y_{m} \theta_{m}\right) \\
\gamma_{2}^{\prime \prime} \equiv \gamma_{2}-\left(x_{1} \delta_{1}+x_{2} \delta_{2}+\cdots+x_{l} \delta_{l}+y_{1} \theta_{1}+y_{2} \theta_{2}+\cdots+y_{m} \theta_{m}\right)
\end{array}\right. \text { or } \\
& \left\{\begin{array}{l}
\gamma_{1}^{\prime \prime} \equiv \gamma_{1}+\left(x_{1} \delta_{1}+x_{2} \delta_{2}+\cdots+x_{l} \delta_{l}+y_{1} \theta_{1}+y_{2} \theta_{2}+\cdots+y_{m} \theta_{m}\right) \\
\gamma_{2}^{\prime \prime} \equiv \gamma_{2}-\left(x_{1} \delta_{1}+x_{2} \delta_{2}+\cdots+x_{l} \delta_{l}+y_{1} \theta_{1}+y_{2} \theta_{2}+\cdots+y_{m} \theta_{m}\right)+n / 2 .
\end{array}\right.
\end{aligned}
$$

(b)

Moreover, we have;

$$
\begin{aligned}
& \left\{\begin{array}{l}
\gamma_{1}^{\prime} \equiv \gamma_{1}^{\prime \prime}+z\left(\gamma_{1}^{\prime \prime}+\gamma_{2}^{\prime \prime}\right) \\
\gamma_{2}^{\prime} \equiv \gamma_{2}^{\prime \prime}-z\left(\gamma_{1}^{\prime \prime}+\gamma_{2}^{\prime \prime}\right) \quad \text { if } \quad \varepsilon=0, \quad \text { or }
\end{array}\right. \\
& \left\{\begin{array}{l}
\gamma_{1}^{\prime} \equiv \gamma_{2}^{\prime \prime}+z\left(\gamma_{1}^{\prime \prime}+\gamma_{2}^{\prime \prime}\right) \\
\gamma_{2}^{\prime} \equiv \gamma_{1}^{\prime \prime}-z\left(\gamma_{1}^{\prime \prime}+\gamma_{2}^{\prime \prime}\right) \text { if } \varepsilon=1 .
\end{array}\right.
\end{aligned}
$$

First, we will prove $\gamma_{1}=\gamma_{1}^{\prime}$ in the case of (i) or (ii), respectively. Next, we will prove $\gamma_{2}=\gamma_{2}^{\prime}$.

In the case of $(i)$, we have $\gamma_{1}^{\prime} \equiv \gamma_{1}^{\prime \prime}+z\left(\gamma_{1}+\gamma_{2}\right) \equiv \gamma_{1}^{\prime \prime}(\bmod \alpha)$. Moreover in the case of $(\mathrm{a})$ or $(\mathrm{b})$, we have $\gamma_{1}^{\prime \prime} \equiv \gamma_{1}(\bmod \alpha)$. Hence we have $\gamma_{1}^{\prime}=\gamma_{1}$, since $0 \leqq \gamma_{1} \leqq[\alpha / 2]$ and $0 \leqq \gamma_{1}^{\prime} \leqq[\alpha / 2]$.

In the case of (ii), we have $\gamma_{1} \equiv \gamma_{1}^{\prime \prime} \equiv \gamma_{2}^{\prime}(\bmod \alpha), \gamma_{2} \equiv \gamma_{2}^{\prime \prime} \equiv \gamma_{1}^{\prime}(\bmod \alpha)$ and $\gamma_{1}+\gamma_{1}^{\prime} \equiv \gamma_{1}+\gamma_{2} \equiv 0(\bmod \alpha)$. Hence we have $\gamma_{1}=\gamma_{1}^{\prime}=0$ or $\gamma_{1}=\gamma_{1}^{\prime}=\alpha / 2$ since $0 \leqq \gamma_{1} \leqq[\alpha / 2]$ and $0 \leqq \gamma_{1}^{\prime} \leqq[\alpha / 2]$.

In the case of (a), we have $\gamma_{1}+\gamma_{2} \equiv \gamma_{1}^{\prime \prime}+\gamma_{2}^{\prime \prime} \equiv \gamma_{1}^{\prime}+\gamma_{2}^{\prime}(\bmod n)$. Since $0 \leqq \gamma_{1}+\gamma_{2} \leqq n / 2$ and $0 \leqq \gamma_{1}^{\prime}+\gamma_{2}^{\prime} \leqq n / 2$, we have $\gamma_{1}+\gamma_{2}=\gamma_{1}^{\prime}+\gamma_{2}^{\prime}$. Hence we have $\gamma_{2}=\gamma_{2}^{\prime}$.

In the case of (b), we have $\gamma_{1}^{\prime}+\gamma_{2}^{\prime} \equiv \gamma_{1}^{\prime \prime}+\gamma_{2}^{\prime \prime} \equiv \gamma_{1}+\gamma_{2}+n / 2(\bmod n)$. By an elementary algebra, we have that $\gamma_{2}=n / 2$ or $\gamma_{2}^{\prime}=n / 2$ which is a contradiction. 
Using the homology group of a covering space of $X$ or by a geometric consideration, we have the following;

Proposition 4.4. (1) An element $(f, M)$ of $P_{n}$ corresponding to an element of $\mathscr{K}_{n}^{-}(2 ; l, m)$ belongs to $P_{n}^{0}$, if $n$ is odd.

(2) An element $(f, M)$ of $P_{n}$ corresponding to an element of $\mathscr{Z}_{n}^{-}(2$; $l, m)^{0}$ or $\mathscr{Z}_{n}^{-}(2 ; l, m)^{*}$ belongs to $P_{n}^{0}$, if either $n$ is even and $d$ is odd, or $n$ is even, $d$ is even and $\gamma=\gamma_{1}+\gamma_{2}$ is odd, where $d=$ g.c.d. $\left\{\delta_{1}, \delta_{2}, \cdots, \delta_{l}\right.$, $\left.\theta_{1}, \theta_{2}, \cdots, \theta_{m}, n\right\}$.

(3) An element of $(f, M)$ of $P_{n}$ corresponding to an element of $\mathscr{Z}_{n}^{-}(2 ; l, m)^{0}$ or $\mathscr{Z}_{n}^{-}(2 ; l, m)^{*}$ belongs to $P_{n}^{-}$, if $n$ is even, $d$ is even and $\gamma=\gamma_{1}+\gamma_{2}$ is even.

\section{§5. Proof of Theorem A.}

In this section, we will give classification of periodic maps on compact non-orientable surfaces. That is, we will obtain the necessary and sufficient conditions that $P_{n}^{0}(\tilde{g}, \tilde{l}, \tilde{m}, \tilde{l}, \tilde{m}) \neq \varnothing$ and determine the number of elements of $\mathscr{P}_{n}^{0}(\widetilde{g}, \tilde{l}, \tilde{m}, \tilde{l}, \tilde{m})$.

For a compact non-orientable surface $M$ and a periodic map $f$ on $M$, using the orbit space $M / f$ and the branched cover $p: M \rightarrow M / f$, we have the following;

Proposition 5.1. If $P_{n}^{0}(\widetilde{g}, \tilde{l}, \tilde{m}, \tilde{l}, \tilde{m}) \neq \varnothing$, then we have

(1) $\tilde{l}=\sum_{a \mid n} \tilde{l}_{a}$ and $\tilde{m}=\sum_{\substack{a \mid n \\ a \neq n}} \tilde{m}_{a}$,

(2) $\widetilde{l}_{a} \equiv 0(\bmod a)$ for each divisor $a$ of $n$ and $\widetilde{m}_{a} \equiv 0(\bmod a)$ for each divisor a of $n$ except $n$.

(3) $\widetilde{g}-2+\sum_{a \mid n}(1-n / a)\left(\tilde{l}_{a}+\tilde{m}_{a}\right)+2 n$ is a positive integer and a multiple of $n$.

Under the conditions (1), (2) and (3) in Proposition 5.1, we will prove Theorem A. We take vectors $\ell=\left(l_{a}\right)_{a \mid n}$ of non-negative integers and $m=\left(m_{a}\right)_{a \mid n}$ of non-negative integers, where $l_{a}=\widetilde{l}_{a} / a$ and $m_{a}=\widetilde{m}_{a} / a$. For $n, \ell$ and $m$ satisfying $l_{n / 2}=m_{n / 2}=0$, we take a set

$$
D(n ; \gamma, m)^{0}=\left\{\begin{array}{l}
\left(\delta_{1}, \delta_{2}, \cdots, \delta_{l}, \theta_{1}, \theta_{2}, \cdots, \theta_{m}\right) ; \\
\text { (i) } 0 \leqq \delta_{1} \leqq \delta_{2} \leqq \cdots \leqq \delta_{l}<\frac{n}{2}, 1 \leqq \theta_{1} \leqq \theta_{2} \leqq \cdots \leqq \theta_{m}<\frac{n}{2} \\
\text { (ii) } l_{a} \text { is the number of elements of the set } \\
\left\{\delta ; \text { g.c.d. }\left\{\delta_{j}, n\right\}=a\right\} \text { and } m_{a} \text { is the number } \\
\text { of elements of the set }\left\{\theta_{k} ; \text { g.c.d. }\left\{\theta_{k}, n\right\}=a\right\}
\end{array}\right\}
$$


where $l=\sum_{a \mid n} l_{a}$ and $m=\sum_{\substack{a \mid n \\ a \neq n}} m_{a}$.

For $n, \downarrow$ and $m$ satisfying that $l_{n / 2}+m_{n, 2} \neq 0$, we take a set

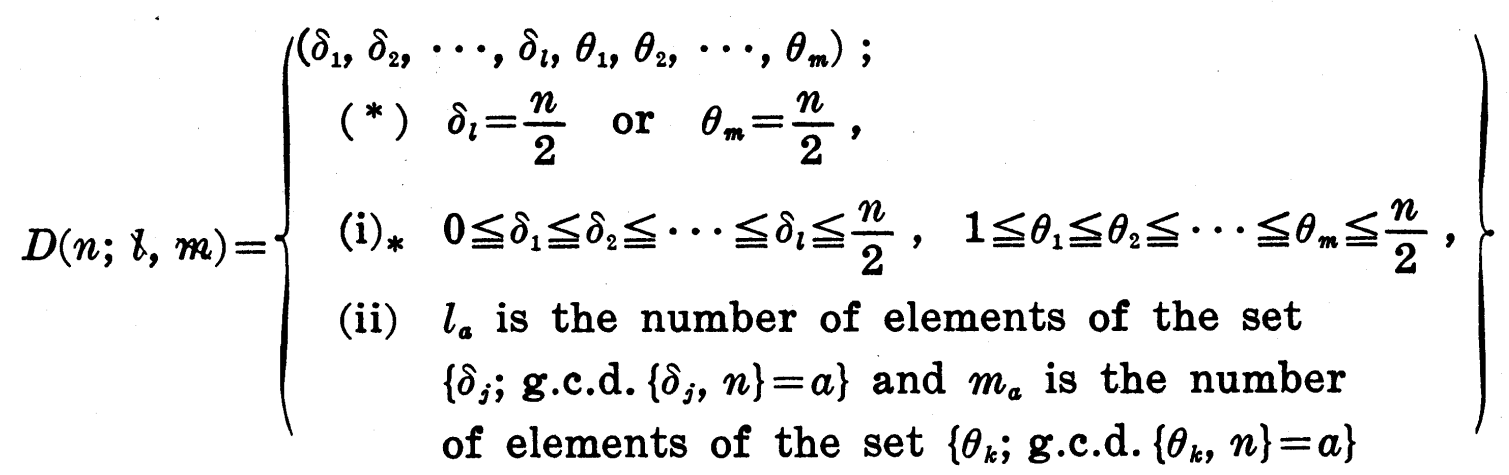

where $l=\sum_{a \mid n} l_{a}$ and $m=\sum_{\substack{a \mid n \\ a \neq n}} m_{a}$.

Then we have clearly;

LEMmA 5.1. For an element of the set $D(n ; \downarrow, m)^{0}$ or the set $D(n ; \chi, m)^{*}$, we have; $\delta_{1}+\delta_{2}+\cdots+\delta_{l}+\theta_{1}+\theta_{2}+\cdots+\theta_{m}$ is even (resp. odd) iff $n$, and $m$ satisfy the condition $\left(5_{c}\right)$ (resp. (5) $)_{0}$, where the condition (5), is that $\sum_{\substack{a \mid n \\ a: \text { odd }}}\left(l_{a}+m_{a}\right)$ is even, and the condition $(5)_{0}$ is that $\sum_{\substack{a ! n \\ a \text { :odd }}}\left(l_{a}+m_{a}\right)$ is odd. Moreover the number of elements of $D(n ; \downarrow, m)^{0}$ or $D(n ; \chi, m)^{*}$ is equal to

$$
C(n ; \varnothing, m)=\prod_{\substack{a \mid n \\
a \neq n \\
a \neq n / 2}}\left(\begin{array}{c}
\frac{\varphi(n / a)}{2}+l_{a}-1 \\
l_{a}
\end{array}\right)\left(\begin{array}{c}
\frac{\varphi(n / a)}{2}+m_{a}-1 \\
m_{a}
\end{array}\right) .
$$

We denote by $D_{e}(n ; \chi, m)^{0} \quad\left(\right.$ resp. $\left.D_{e}(n ; \chi, m)^{*}\right)$ the set $D(n ; \chi, m)^{0}$ (resp. $D(n ; \chi, m)^{*}$ ) when $n, \downarrow$ and $m$ satisfy the condition $(5)_{e}$, and by $D_{o}(n ; \chi, m)^{0}\left(\operatorname{resp} . D_{o}(n ; \chi, m)^{*}\right)$ the set $D(n ; \chi, m)^{0}\left(\operatorname{resp} . D(n ; \chi, m)^{*}\right)$ when $n, \downarrow$ and $m$ satisfy the condition $(5)_{o}$.

Let $g=(1 / n)\left\{\widetilde{g}-2+\sum_{a \mid n}(1-n / a)\left(\widetilde{l}_{a}+\tilde{m}_{a}\right)+2 n\right\}$. We will prove Theorem $A$ in respective cases.

PROOF IN CASE THAT $g$ IS ODD AND $g \geqq 3$. (I) Suppose that $n$ is odd. Then there is a bijection of $\mathscr{P}_{n}^{0}(\widetilde{g}, \widetilde{l}, \tilde{m}, \tilde{l}, \tilde{m})$ onto the subset $\mathscr{\varkappa}_{n}^{-}(g ; l, m, \chi, m)$ of $\mathscr{Z}_{n}^{-}(g ; l, m)$ satisfying the condition (ii), where the condition (ii) is that $l_{a}$ is the number of elements of the set $\left\{\delta_{j} ;\right.$ g.c.d. $\left.\left\{\delta_{j}, n\right\}=a\right\}$ and $m_{a}$ is the number of elements of the set $\left\{\theta_{k} ;\right.$ g.c.d. $\left.\left\{\theta_{k}, n\right\}=a\right\}$. For any element $\left(\delta_{1}, \delta_{2}, \cdots, \delta_{l}, \theta_{1}, \theta_{2}, \cdots, \theta_{m}\right)$ of $D(n ; \chi, m)^{0}$, there is exactly one non-negative integer $\gamma$ such that $\left(1,0, \cdots, 0,0, \gamma, \delta_{1}, \delta_{2}, \cdots, \delta_{l}, \theta_{1}, \theta_{2}\right.$, $\left.\cdots, \theta_{m}\right) \in \mathscr{\varkappa}_{n}^{-}(g ; l, m, \ell, m)$. Hence there is a bijection of $\mathscr{\varkappa}_{n}^{-}(g ; l, m, \ell, m)$ onto $D(n ; \Varangle, m)^{0}$. By Lemma 5.1 , we have the proof. 
(II) Suppose that $n$ is even. If $l_{n / 2}=m_{n / 2}=0$, there is a bijection of $\mathscr{F}_{n}^{0}(\widetilde{g}, \widetilde{l}, \widetilde{m}, \tilde{\mathcal{Z}}, \tilde{\mathfrak{R}})$ onto the subset $\mathscr{Z}_{n}^{-}(g ; l, m, \mathcal{Z}, m)_{1}^{0}$ of $\mathscr{Z}_{n}^{-}(g ; l, m)_{1}^{0}$ satisfying the condition (ii). If $l_{n / 2}+m_{n / 2} \neq 0$, there is a bijection of $\mathscr{P}_{n}^{0}(\widetilde{g}, \tilde{l}$, $\tilde{m}, \tilde{l}, \tilde{m})$ onto the subset $\mathscr{\varkappa}_{n}^{-}(g ; l, m, \ell, m)_{1}^{*}$ of $\mathscr{\varkappa}_{n}^{-}(g ; l, m)_{1}^{*}$ satisfying the condition (ii). For any element $\left(\delta_{1}, \delta_{2}, \cdots, \delta_{l}, \theta_{1}, \theta_{2}, \cdots, \theta_{m}\right)$ of $D_{c}(n ; \ell, m)^{0}$, there are exactly two non-negative integers $\gamma$ satisfying that $(1,0, \cdots$, $\left.0,0, \gamma, \delta_{1}, \delta_{2}, \cdots, \delta_{l}, \theta_{1}, \theta_{2}, \cdots, \theta_{m}\right) \in \mathscr{\varkappa}_{n}^{-}(g ; l, m, \ell, m)_{1}^{0}$. For any element $\left(\delta_{1}\right.$, $\left.\delta_{2}, \cdots, \delta_{l}, \theta_{1}, \theta_{2}, \cdots, \theta_{m}\right)$ of $D_{b}(n ; \gamma, m)^{*}$, there is exactly one non-negative integer $\gamma$ satisfying that $\left(1,0, \cdots, 0,0, \gamma, \delta_{1}, \delta_{2}, \cdots, \delta_{l}, \theta_{1}, \theta_{2}, \cdots, \theta_{m}\right) \in \mathscr{Z}_{n}^{-}(g$; $l, m, \ell, m)_{1}^{*}$. For any element $\left(\delta_{1}, \delta_{2}, \cdots, \delta_{l}, \theta_{1}, \theta_{2}, \cdots, \theta_{m}\right)$ of $D_{0}(n ; \ell, m)^{0}$ (resp. $\left.D_{o}(n ; \gamma, m)^{*}\right)$, there are no non-negative integers $\gamma$ satisfying that $\left(1,0, \cdots, 0,0, \gamma, \delta_{1}, \delta_{2}, \cdots, \delta_{l}, \theta_{1}, \theta_{2}, \cdots, \theta_{m}\right) \in \mathscr{Z}_{n}^{-}(g ; l, m, \gamma, m)_{1}^{0}$ (resp. $\mathscr{Z}_{n}^{-}(g$; $\left.l, m, \downarrow, m)_{1}^{*}\right)$. Hence, by Lemma 5.1, we have the proof.

Proof IN CASE THAT $g=1$. By Proposition 2.4, we may consider only when $n, l$ and $m$ satisfy the condition that $d=$ g.c.d. $\left\{a ; l_{a} \neq 0\right.$ or $\left.m_{a} \neq 0\right\}$ is odd. If $n$ is odd, then there is a bijection of $\mathscr{P}_{n}^{0}(\widetilde{g}, \tilde{l}, \tilde{m}, \tilde{b}, \tilde{m})$ onto the subset $\mathscr{Z}_{n}^{-}(1 ; l, m, \ell, m)$ of $\mathscr{Z}_{n}^{-}(1 ; l, m)$ satisfying the condition (ii). Since $2 \gamma+\delta_{1}+\delta_{2}+\cdots+\delta_{l}+\theta_{1}+\theta_{2}+\cdots+\theta_{m} \equiv 0(\bmod n)$, we have $2 \gamma \equiv 0(\bmod$ $d)$. Hence we have $\gamma \equiv 0(\bmod d)$, that is, $\gamma$ is a multiple of $d$. Therefore g.c.d. $\left\{\gamma, \delta_{1}, \delta_{2}, \cdots, \delta_{l}, \theta_{1}, \theta_{2}, \cdots, \theta_{m}, n\right\}=1$ iff $d=$ g.c.d. $\left\{a ; l_{a} \neq 0\right.$ or $\left.m_{a} \neq 0\right\}=$ g.c.d. $\left\{\delta_{1}, \delta_{2}, \cdots, \delta_{l}, \theta_{1}, \theta_{2}, \cdots, \theta_{m}, n\right\}=1$. Adding the condition that g.c.d. $\left\{a ; l_{a} \neq 0\right.$ or $\left.m_{a} \neq 0\right\}=1$, we complete the proof by the same way as in case that $g$ is odd and $g \geqq 3$.

Proof IN CASE THAT $g$ is EVEN AND $g \geqq 4$.

By the same way as the proof in case that $g$ is odd and $g \geqq 3$, we complete the proof.

Proof IN CASE that $g=2$. Let $\left(\delta_{1}, \delta_{2}, \cdots, \delta_{l}, \theta_{1}, \theta_{2}, \cdots, \theta_{m}\right)$ be an element of $D(n ; \ell, m)^{0}$ or $D(n ; \gamma, m)^{*}$. Let $d=$ g.c.d. $\left\{\delta_{1}, \delta_{2}, \cdots, \delta_{l}, \theta_{1}, \theta_{2}\right.$, $\left.\cdots, \theta_{m}, n\right\}=$ g.c.d. $\left\{a ; l_{a} \neq 0\right.$ or $\left.m_{a} \neq 0\right\}$. Let $\gamma$ be a non-negative integer such that $2 \gamma+\delta_{1}+\delta_{2}+\cdots+\delta_{l}+\theta_{1}+\theta_{2}+\cdots+\theta_{m} \equiv 0(\bmod n)$.

Let $\mathscr{Z}_{n}^{-}(2 ; l, m, \downarrow, m)^{0}$ (resp. $\left.\mathscr{\varkappa}_{n}^{-}(2 ; l, m, \downarrow, m)^{*}\right)$ be the subset of $\mathscr{Z}_{n}^{-}(2 ; l, m)^{0}$ (resp. $\left.\mathscr{Z}_{n}^{-}(2 ; l, m)^{*}\right)$ satisfying the condition (ii). If $d$ is odd, we have $\gamma \equiv 0(\bmod d)$. That is, there exists a non-negative integer $i$ such that $\gamma=i d$. For a non-negative integer $\gamma=i d$, there are exactly $\{\varphi(d) / 2\}$ non-negative integers $\gamma_{1}$ satisfying that $\gamma=\gamma_{1}+\gamma_{2}$ and $\left(\gamma_{1}, \gamma_{2}, \delta_{1}\right.$, $\left.\delta_{2}, \cdots, \delta_{l}, \theta_{1}, \theta_{2}, \cdots, \theta_{m}\right) \in \mathscr{Z}_{n}^{-}(2 ; l, m, \ell, m)^{0}\left(\right.$ resp. $\left.\mathscr{Z}_{n}^{-}(2 ; l, m, \ell, m)^{*}\right)$, since g.c.d. $\left\{\gamma_{1}, \gamma_{2}, d\right\}=$ g.c.d. $\left\{\gamma_{1}, d\right\}=1$ and $0 \leqq \gamma_{1} \leqq[\alpha / 2]=[d / 2]=(d-1) / 2$.

Let $\mathscr{Z}_{n}^{-}(2 ; l, m, \ell, m)_{12}^{0}$ (resp. $\left.\mathscr{Z}_{n}^{-}(2 ; l, m, \ell, m)_{12}^{*}\right)$ be the subset of 
$\mathscr{Z}_{n}^{-}(2 ; l, m)^{0}\left(\right.$ resp. $\left.\mathscr{K}_{n}^{-}(2 ; l, m)^{*}\right)$ satisfying the condition (ii) and that $\gamma$ is odd. If $d$ is even and $\gamma$ is odd, we have $\gamma \equiv 0(\bmod d / 2)$. We note that if $n / 2$ is even, then $\gamma$ is not odd. If $n / 2$ is odd, then there exists a non-negative integer $i$ such that $\gamma=i d+d / 2$. For an odd positive integer $\gamma=i d+d / 2$, there are exactly $\{\phi(d / 2) / 2\}$ non-negative integers $\gamma_{1}$ satisfying that $\gamma=\gamma_{1}+\gamma_{2}$ and $\left(\gamma_{1}, \gamma_{2}, \delta_{1}, \delta_{2}, \cdots, \delta_{l}, \theta_{1}, \theta_{2}, \cdots, \theta_{m}\right) \in \mathscr{Z}_{n}^{-}(2 ; l$, $m, \ell, m)_{12}^{0}$ (resp. $\mathscr{Z}_{n}^{-}(2 ; l, m, \ell, m)_{12}^{*}$ ), since g.c.d. $\left\{\gamma_{1}, \gamma_{2}, d\right\}=$ g.c.d. $\left\{\gamma_{1}, d / 2\right\}=1$ and $0 \leqq \gamma_{1} \leqq[\alpha / 2]=[d / 4]$.

( I ) Suppose that $n$ is odd. Then, there is a bijection of $\mathscr{P}_{n}^{0}(\widetilde{g}, \tilde{l}$, $\tilde{m}, \tilde{l}, \tilde{m})$ onto $\mathscr{Z}_{n}^{-}(2 ; l, m, \ell, m)^{0}$. For any element $\left(\delta_{1}, \delta_{2}, \cdots, \delta_{l}, \theta_{1}, \theta_{2}, \cdots\right.$, $\left.\theta_{m}\right)$ of $D(n ; \ell, m)^{0}$, there is exactly one non-negative integer in the set $\{\gamma=i d ; 0 \leqq i \leqq n / d\}$ which satisfies $2 \gamma+\delta_{1}+\delta_{2}+\cdots+\delta_{l}+\theta_{1}+\theta_{2}+\cdots+\theta_{m} \equiv 0$ $(\bmod n)$, since $d$ is odd. Hence we have $P_{n}^{0}(\tilde{g}, \tilde{l}, \tilde{m}, \tilde{\boldsymbol{l}}, \tilde{\boldsymbol{m}}) \neq \varnothing$ and the number of elements of $\mathscr{P}_{n}^{0}(\widetilde{g}, \tilde{l}, \tilde{m}, \tilde{l}, \tilde{m})$ is equal to $\{\phi(d) / 2\} \times C(n ; \gamma, m)$.

(II) Suppose that $n$ is even and $d$ is odd. If $l_{n / 2}=m_{n / 2}=0$, there is a bijection of $\mathscr{P}_{n}^{\circ}(\widetilde{g}, \tilde{l}, \tilde{m}, \tilde{l}, \tilde{m})$ onto $\mathscr{z}_{n}^{-}(2 ; l, m, \downarrow, m)^{0}$. For any element $\left(\delta_{1}, \delta_{2}, \cdots, \delta_{l}, \theta_{1}, \theta_{2}, \cdots, \theta_{m}\right)$ of $D_{e}(n ; \ell, m)^{0}$, there are exactly two non-negative integers in the set $\{\gamma=i d ; 0 \leqq i \leqq n / d\}$ which satisfy $2 \gamma+\delta_{1}+\delta_{2}+\cdots+\delta_{l}+$ $\theta_{1}+\theta_{2}+\cdots+\theta_{m} \equiv 0(\bmod n)$. For any element $\left(\delta_{1}, \delta_{2}, \cdots, \delta_{l}, \theta_{1}, \theta_{2}, \cdots, \theta_{m}\right)$ of $D_{o}(n ; \gamma, m)^{0}$, there are no non-negative integers $\gamma$ satisfying that $2 \gamma+\delta_{1}+\delta_{2}+\cdots+\delta_{l}+\theta_{1}+\theta_{2}+\cdots+\theta_{m} \equiv 0(\bmod n)$. If $l_{n / 2}+m_{n / 2} \neq 0$, there is a bijection of $\mathscr{P}_{n}^{\circ}(\widetilde{g}, \widetilde{l}, \tilde{m}, \tilde{l}, \tilde{m})$ onto $\mathscr{Z}_{n}^{-}(2 ; l, m, \mathcal{\ell}, m)^{*}$. For any element $\left(\delta_{1}, \delta_{2}, \cdots, \delta_{l}, \theta_{1}, \theta_{2}, \cdots, \theta_{m}\right)$ of $D_{e}(n ; \chi, m)^{*}$, there is exactly one non-negative integer in the set $\{\gamma=i d ; 0 \leqq i \leqq n / 2 d\}$ which satisfies $2 \gamma+\delta_{1}+\delta_{2}+\cdots+$ $\delta_{l}+\theta_{1}+\theta_{2}+\cdots+\theta_{m} \equiv 0(\bmod n)$. For any element $\left(\delta_{1}, \delta_{2}, \cdots, \delta_{l}, \theta_{1}, \theta_{2}, \cdots, \theta_{m}\right)$ of $D_{0}\left(n ; \mathcal{Z}, m_{2}\right)^{*}$, there are no non-negative integers $\gamma$ satisfying that $2 \gamma+\delta_{1}+\delta_{2}+\cdots+\delta_{l}+\theta_{1}+\theta_{2}+\cdots+\theta_{m} \equiv 0(\bmod n)$. Hence we have $P_{n}^{0}(\widetilde{g}, \widetilde{l}$, $\tilde{m}, \tilde{l}, \tilde{m}) \neq \varnothing$ iff $n, \ell$ and $m$ satisfy the condition (5)。 in Lemma 5.1. Then the number of elements of $\mathscr{P}_{n}^{\circ}(\widetilde{g}, \tilde{l}, \tilde{m}, \tilde{l}, \tilde{m})$ is equal to

$$
\left\{\begin{array}{lll}
2 \times\left\{\frac{\varphi(d)}{2}\right\} \times C(n ; \not, m) & \text { if } & l_{n / 2}=m_{n / 2}=0, \\
\left\{\frac{\varphi(d)}{2}\right\} \times C(n ; \ell, m) & \text { if } & l_{n / 2}+m_{n / 2} \neq 0 .
\end{array}\right.
$$

(III) Suppose that $n$ is even and $d$ is even. If $n / 2$ is odd, there is a bijection of $\mathscr{P}_{n}^{0}(\widetilde{g}, \widetilde{l}, \widetilde{m}, \tilde{z}, \widetilde{m})$ onto $\mathscr{Z}_{n}^{-}(2 ; l, m, \mathcal{Z}, m)_{12}^{0}$. For any element $\left(\delta_{1}, \delta_{2}, \cdots, \delta_{l}, \theta_{1}, \theta_{2}, \cdots, \theta_{m}\right)$ of $D(n ; \ell, m)^{0}$, there is exactly one odd positive integer in the set $\{\gamma=i d+d / 2 ; 0 \leqq i<n / d\}$ which satisfies $2 \gamma+\delta_{1}+\delta_{2}+\cdots+$ $\delta_{l}+\theta_{1}+\theta_{2}+\cdots+\theta_{m} \equiv 0(\bmod n)$. Hence we have $P_{n}^{0}(\widetilde{g}, \widetilde{l}, \tilde{m}, \tilde{l}, \widetilde{m}) \neq \varnothing$, and the number of elements of $\mathscr{\mathscr { P }}_{n}^{0}(\widetilde{g}, \tilde{l}, \tilde{m}, \tilde{l}, \tilde{m})$ is equal to $\{\varphi(d / 2) / 2\} \times$ 
$C(n ; \ell, m)$.

(IV) Suppose that $n$ is even, $d$ is even and $n / 2$ is even. If $l_{n / 2}=$ $m_{n / 2}=0$, there is a bijection of $\mathscr{P}_{n}^{0}(\widetilde{g}, \tilde{l}, \tilde{m}, \tilde{z}, \tilde{m})$ onto $\mathscr{Z}_{n}^{-}(2 ; l, m, \mathcal{Z}, m)_{12}^{0}$. If $l_{n / 2}+m_{n / 2} \neq 0$, there is a bijection of $\mathscr{P}_{n}^{0}(\tilde{g}, \tilde{l}, \tilde{m}, \tilde{\mathbb{l}}, \tilde{m})$ onto $\mathscr{\varkappa}_{n}^{-}(2 ; l, m$, $\gamma, m)_{12}^{*}$. If $d / 2$ is even, there are no odd positive integers $\gamma$ satisfying that $2 \gamma+\delta_{1}+\delta_{2}+\cdots+\delta_{l}+\theta_{1}+\theta_{2}+\cdots+\theta_{m} \equiv 0(\bmod n)$. Hence we have $P_{n}^{0}(\widetilde{g}, \tilde{l}, \tilde{m}, \tilde{l}, \tilde{m})=\varnothing$. We assume that $n, \chi$ and $m$ satisfy the condition (d), where the condition (d) is that there is an even divisor $a$ of $n$ such that $d / 2$ is odd and $l_{a} \neq 0$ or $m_{a} \neq 0$ (i.e. $d / 2$ is odd). For any element $\left(\delta_{1}, \delta_{2}, \cdots \delta_{l}, \theta_{1}, \theta_{2}, \cdots, \theta_{m}\right)$ of $D(n ; \ell, m)^{0}$ (resp. $\left.D(n ; \ell, m)^{*}\right)$ such that $(1 / d)\left(\delta_{1}+\delta_{2}+\cdots+\delta_{l}+\theta_{1}+\theta_{2}+\cdots+\theta_{m}\right)$ is odd, there are exactly two (resp. one) odd positive integers in the set $\{\gamma=i d+d / 2 ; 0 \leqq i<n / d\}$ which satisfy $2 \gamma+\delta_{1}+\delta_{2}+\cdots+\delta_{l}+\theta_{1}+\theta_{2}+\cdots+\theta_{m} \equiv 0(\bmod n)$. For any element $\left(\delta_{1}\right.$, $\left.\delta_{2}, \cdots, \delta_{l}, \theta_{1}, \theta_{2}, \cdots, \theta_{m}\right)$ of $D(n ; \ell, m)^{0} \cup D(n ; \ell, m)^{*}$ such that $(1 / d)\left(\delta_{1}+\right.$ $\left.\delta_{2}+\cdots+\delta_{l}+\theta_{1}+\theta_{2}+\cdots+\theta_{m}\right)$ is even, there are no odd positive integers $\gamma$ satisfying that $2 \gamma+\delta_{1}+\delta_{2}+\cdots+\delta_{l}+\theta_{1}+\theta_{2}+\cdots+\theta_{m} \equiv 0 .(\bmod n)$. We note $(1 / d)\left(\delta_{1}+\delta_{2}+\cdots+\delta_{l}+\theta_{1}+\theta_{2}+\cdots+\theta_{m}\right)$ is odd iff $(1 / 2)\left(\delta_{1}+\delta_{2}+\cdots+\delta_{l}+\right.$ $\left.+\theta_{1}+\theta_{2}+\cdots+\theta_{m}\right)$ is odd. Hence we have $P_{n}^{0}(\widetilde{g}, \tilde{l}, \tilde{m}, \tilde{l}, \tilde{m}) \neq \varnothing$ iff $n, \&$ $m$ satisfy the conditions (d) and (6), where the condition $(6)_{0}$ is that $\sum_{\substack{a|n \\ a \\ a| \text { ieven } \\ a \mid 2: \text { odd }}}\left(l_{a}+m_{a}\right)$ is odd. Then the number of elements of $\mathscr{P}_{n}^{0}(\tilde{g}, \widetilde{l}, \tilde{m}, \tilde{z}, \tilde{m})$ is equal to

$$
\left\{\begin{array}{lll}
2 \times\left\{\frac{\varphi(d / 2)}{2}\right\} \times C(n ; \downarrow, m) & \text { if } & l_{n / 2}=m_{n / 2}=0 \\
\left\{\frac{\varphi(d / 2)}{2}\right\} \times C(n ; \downarrow, m) & \text { if } & l_{n / 2}+m_{n / 2} \neq 0 .
\end{array}\right.
$$

\section{§6. Proof of Theorem B.}

In this section, we will give classification of orientation reversing periodic maps on compact orientable surfaces. That is, we will obtain the necessary and sufficient conditions that $P_{n}^{-}(\widetilde{g}, \tilde{l}, \tilde{m}, \tilde{l}, \tilde{m}) \neq \varnothing$, and determine the number of elements of $\mathscr{P}_{n}^{-}(\widetilde{g}, \widetilde{l}, \widetilde{m}, \tilde{l}, \widetilde{m})$.

Using the orbit space $M / f$ and the branched cover $p: M \rightarrow M / f$ and by Propositions 2.4, 3.4 and 4.4, we have the following;

Proposition 6.1. If $P_{n}^{-}(\tilde{g}, \tilde{l}, \tilde{m}, \tilde{b}, \tilde{m}) \neq \varnothing$, then we have

(0) $n$ is even,

(1) $\widetilde{l}=\sum_{a \mid n} \widetilde{l}_{a}$ and $\tilde{m}=\sum_{\substack{a \mid n \\ a \neq n}} \widetilde{m}_{a}$,

(2) $\tilde{l}_{a} \equiv 0(\bmod a)$ for each divisor $a$ of $n$ and $\tilde{m}_{a} \equiv 0(\bmod a)$ for each 
divisor $a$ of $n$ except $n$,

$(3)^{\prime} 2 \widetilde{g}-2+\sum_{a \mid n}(1-n / a)\left(\tilde{l}_{a}+\tilde{m}_{a}\right)+2 n$ is a positive integer and $a$ multiple of $2 n$.

(4) $\widetilde{l}_{a}=\tilde{m}_{a}=0$ for each odd divisor $a$ of $n$.

Under the conditions (0), (1), (2), (3)' and (4) in Proposition 6.1, we will prove Theorem $B$ by the similar way as in $\S 5$. We take vectors $\chi=\left(l_{a}\right)_{a \mid n}$ of non-negative integers and $m=\left(m_{a}\right)_{a \mid n}$ of non-negative integers, where $l_{a}=\tilde{l}_{a} / a$ and $m_{a}=\tilde{m}_{a} / a$. Let $D(n ; \chi, m)^{0}$ and $D(n ; \chi, m)^{*}$ be the same sets as in $\S 5$. We denote by $D^{-}(n ; \chi, m)^{0}\left(\operatorname{resp.} D^{-}(n ; \chi, m)^{*}\right)$ the set $D(n ; \chi, m)^{0}$ (resp. $\left.D(n ; \chi, m)^{*}\right)$ when $n, \downarrow$ and $m$ satisfy the condition (4).

Then we have clearly;

LEMMA 6.1. ( $n$; even) For an element of the set $D^{-}(n ; \chi, m)^{0}$ or the set $D^{-}(n ; \chi, m)^{*}$, we have; $(1 / 2)\left(\delta_{1}+\delta_{2}+\cdots+\delta_{l}+\theta_{1}+\theta_{2}+\cdots+\theta_{m}\right)$ is even (resp. odd) iff $n$, and $m$ satisfy the conditon (6) (resp. (6)o), where the condition (6). is that $\sum_{\substack{a \mid n \\ a: \text { even } \\ a: 2: \text { odd }}}\left(l_{a}+m_{a}\right)$ is even, and the condition (6)。 is that $\sum_{\substack{a \mid n \\ a: \text { eren } \\ \text { a:2:odd }}}\left(l_{a}+m_{a}\right)$ is odd.

Moreover the number of elements of $D(n ; \chi, m)^{0}$ or $D(n ; \chi, m) *$ is equal to $C(n ; \chi, m)$.

We denote by $D_{e}^{-}(n ; \gamma, m)^{0}\left(\operatorname{resp} . D_{e}^{-}(n ; \gamma, m)^{*}\right)$ the set $D^{-}(n ; \gamma, m)^{0}$ (resp. $\left.D^{-}(n ; \chi, m)^{*}\right)$ when $n, \downarrow$ and $m$ satisfy the condition $(6)_{e}$; and by $D_{o}^{-}(n ; \chi, m)^{0}\left(\operatorname{resp} . D_{o}^{-}(n ; \chi, m)^{*}\right)$ the set $D^{-}(n ; \gamma, m)^{0}\left(\operatorname{resp} . D^{-}(n ; \chi, m)^{*}\right)$ when $n, \downarrow$ and $m$ satisfy the condition $(6)_{o}$.

Let $g=(1 / 2 n)\left\{2 \widetilde{g}+\sum_{a \mid n}(1-2 n / a)\left(\chi_{a}+m_{a}\right)+2 n-2\right\}$. We will prove Theorem $B$ in respective cases.

ProOF IN CASE THAT $g$ IS ODD AND $g \geqq 3$. (I) Suppose that $n / 2$ is odd. Then there is a bijection of $\mathscr{P}_{n}^{-}(\widetilde{g}, \tilde{l}, \tilde{m}, \tilde{l}, \tilde{m})$ onto the subset $\mathscr{Z}_{n}^{-}(g ; l, m, \tilde{\chi}, \tilde{\mathscr{M}})_{2}^{0}$ of $\mathscr{Z}_{n}^{-}(g ; l, m)_{2}^{0}$ satisfying the condition (ii), where the condition (ii) is that $l_{a}$ is the number of elements of the set $\left\{\delta_{j}\right.$; g.c.d. $\left.\left\{\delta_{j}, n\right\}=a\right\}$ and $m_{a}$ is the number of elements of the set $\left\{\theta_{k}\right.$; g.c.d. $\left\{\theta_{k}, n\right\}=$ a\}. By the same argument as the proof in $\S 5$, there is a bijection of $\mathscr{K}_{n}^{-}(g ; l, m, \downarrow, m)_{2}^{0}$ onto $D^{-}(n ; \downarrow, m)^{0}$. By Lemma 6.1 , we have the proof.

(II) Suppose that $n / 2$ is even. If $l_{n / 2}=m_{n / 2}=0$, then there is a bijection of $\mathscr{P}_{n}^{-}(\tilde{g}, \tilde{l}, \tilde{m}, \tilde{l}, \tilde{\mathscr{R}})$ onto $\mathscr{K}_{n}^{-}(g ; l, m, \ell, m)_{2}^{0}$. If $l_{n / 2}+m_{n / 2} \neq 0$, then there is a bijection of $\mathscr{P}_{n}^{-}(\widetilde{g}, \tilde{l}, \tilde{m}, \tilde{l}, \tilde{m})$ onto the subset $\mathscr{\varkappa}_{n}^{-}(g ; l, m, \chi, m)_{2}^{*}$ 
of $\mathscr{Z}_{n}^{-}(g ; l, m)_{2}^{*}$ satisfying the condition (ii).

For any element $\left(\delta_{1}, \delta_{2}, \cdots, \delta_{l}, \theta_{1}, \theta_{2}, \cdots, \theta_{m}\right)$ of $D_{0}^{-}\left(n ; \ell, m_{2}\right)^{0}$ (resp. $\left.D_{0}^{-}(n ; \gamma, m)^{*}\right)$ there are exactly two (resp. one) non-negative integers $\gamma$ such that $\left(2,0, \cdots, 0,0, \gamma, \delta_{1}, \delta_{2}, \cdots, \delta_{l}, \theta_{1}, \theta_{2}, \cdots, \theta_{m}\right) \in \mathscr{Z}_{n}^{-}\left(g ; l, m, \mathcal{Z}, m_{2}^{0}\right.$ (resp. $\left.\mathscr{Z}_{n}^{-}(g ; l, m, \ell, m)_{2}^{*}\right)$. For any element $\left(\delta_{1}, \delta_{2}, \cdots, \delta_{l}, \theta_{1}, \theta_{2}, \cdots, \theta_{m}\right)$ of $D_{e}^{-}(n ; \gamma, m)^{0}$ (resp. $\left(D_{e}^{-}(n ; \gamma, m)^{*}\right)$, there are no non-negative integers $\gamma$ such that $\left(2,0, \cdots, 0,0, \gamma, \delta_{1}, \delta_{2}, \cdots, \delta_{l}, \theta_{1}, \theta_{2}, \cdots, \theta_{m}\right) \in \mathscr{Z}_{n}^{-}(g ; l, m, \downarrow, m)_{2}^{0}$ (resp. $\left(\mathscr{K}_{n}^{-}(g ; l, m, \downarrow, m)_{2}^{*}\right)$. By Lemma 6.1, we have the proof.

Proof IN CASE THAT $g=1$. By Proposition 2.4, we may consider only when $n, \&$ and $m$ satisfy that $d=$ g.c.d. $\left\{a ; l_{a} \neq 0\right.$ or $\left.m_{a} \neq 0\right\}$ is even. Let $d^{\prime}=(1 / 2)$ g.c.d. $\left\{\delta_{1}, \delta_{2}, \cdots, \delta_{l}, \theta_{1}, \theta_{2}, \cdots, \theta_{m}, n\right\}=(1 / 2)$ g.c.d. $\left\{a ; l_{a} \neq 0\right.$ or $\left.m_{a} \neq 0\right\}$. Since $2 \gamma+\delta_{1}+\delta_{2}+\cdots+\delta_{l}+\theta_{1}+\theta_{2}+\cdots+\theta_{m} \equiv 0(\bmod n)$, we have $\gamma+\delta_{1} / 2+$ $\delta_{2} / 2+\cdots+\delta_{l} / 2+\theta_{1} / 2+\theta_{2} / 2+\cdots+\theta_{m} / 2 \equiv 0(\bmod n / 2)$. Hence $d^{\prime}$ is a divisor of $\gamma$. Therefore g.c.d. $\left\{\gamma, \delta_{1}, \delta_{2}, \cdots, \delta_{l}, \theta_{1}, \theta_{2}, \cdots, \theta_{m}, n\right\}=1$ iff $d^{\prime}=1$. Adding the condition that (1/2) g.c.d. $\left\{a ; l_{a} \neq 0\right.$ or $\left.m_{a} \neq 0\right\}=1$, we complete the proof by the same way as in case that $g$ is odd and $g \geqq 3$.

Proof in Case that $g$ is even and $g \geqq 4$. By the same way as the proof in case that $g$ is odd and $g \geqq 3$, we complete the proof.

Proof IN CASE That $g=2$. Let $\left(\delta_{1}, \delta_{2}, \cdots, \delta_{l}, \theta_{1}, \theta_{2}, \cdots, \theta_{m}\right)$ be an element of $D^{-}(n ; \ell, m)^{0}$ or $D^{-}(n ; \gamma, m)^{*}$. Let $d=$ g.c.d. $\left\{\delta_{1}, \delta_{2}, \cdots, \delta_{l}, \theta_{1}, \theta_{2}\right.$, $\left.\cdots, \theta_{m}, n\right\}=$ g.c.d. $\left\{a ; l_{a} \neq 0\right.$ or $\left.m_{a} \neq 0\right\}$. Let $\gamma$ be a non-negative integer such that $2 \gamma+\delta_{1}+\delta_{2}+\cdots+\delta_{l}+\theta_{1}+\theta_{2}+\cdots+\theta_{m} \equiv 0(\bmod n)$. Let $\mathscr{Z}_{n}^{-}(2 ; l, m, \mathcal{\gamma}, m)_{2}^{0}$ (resp. $\left.\mathscr{Z}_{n}^{-}(2 ; l, m, \ell, m)_{2}^{*}\right)$ be the subset of $\mathscr{Z}_{n}^{-}(2 ; l, m)^{0}\left(\operatorname{resp} . \mathscr{Z}_{n}^{-}(2 ; l, m)^{*}\right)$ satisfying the condition (ii) and satisfying that $\gamma$ is even. If $d$ is even, we have $\gamma \equiv 0(\bmod d / 2)$. Hence there exists a non-negative integer $i$ such that $\gamma=i d$ or $\gamma=i d+d / 2$. For an even non-negative integer $\gamma=i d$, there are exactly $\{\varphi(d) / 2\}$ non-negative integers $\gamma_{1}$ such that $\gamma=\gamma_{1}+\gamma_{2}$ and $\left(\gamma_{1}, \gamma_{2}, \delta_{1}, \delta_{2}, \cdots, \delta_{l}, \theta_{1}, \theta_{2}, \cdots, \theta_{m}\right) \in \mathscr{Z}_{n}^{-}(2 ; l, m, \chi, m)_{2}^{0}\left(\operatorname{resp} . \mathscr{Z}_{n}^{-}(2 ; l, m, \ell, m)_{2}^{*}\right)$, since g.c.d. $\left\{\gamma_{1}, \gamma_{2}, d\right\}=$ g.c.d. $\left\{\gamma_{1}, d\right\}=1$, and $0 \leqq \gamma_{1} \leqq[\alpha / 2]=d / 2$. For an even positive integer $\gamma=i d+d / 2$, there are exactly $\{\varphi(d / 2) / 2\}$ non-negative integers $\gamma_{1}$ such that $\gamma=\gamma_{1}+\gamma_{2}$ and $\left(\gamma_{1}, \gamma_{2}, \delta_{1}, \delta_{2}, \cdots, \delta_{l}, \theta_{1}, \theta_{2}, \cdots \theta_{m}\right) \in \mathscr{Z}_{n}^{-}(2$; $l, m, \ell, m)_{2}^{0}\left(\right.$ resp. $\left.\mathscr{Z}_{n}^{-}(2 ; l, m, \ell, m)_{2}^{*}\right)$, since g.c.d. $\left\{\gamma_{1}, \gamma_{2}, d\right\}=$ g.c.d. $\left\{\gamma_{1}, d / 2\right\}=$ 1 , and $0 \leqq \gamma_{1} \leqq[\alpha / 2]=[d / 4]$.

(I) Suppose that $n / 2$ is odd. Then there is a bijection of $\mathscr{P}_{n}^{-}(\widetilde{g}, \tilde{l}$, $\tilde{m}, \tilde{l}, \tilde{m})$ onto $\mathscr{Z}_{n}^{-}(2 ; l, m, \mathcal{\ell}, m)_{2}^{0}$. For any element $\left(\delta_{1}, \delta_{2}, \cdots, \delta_{l}, \theta_{1}, \theta_{2}, \cdots\right.$, $\left.\theta_{m}\right)$ of $D^{-}(n ; \ell, m)^{0}$, there is exactly one even non-negative integer in the set $\{\gamma=i d ; 0 \leqq i \leqq n / d\}$ which satisfies $2 \gamma+\delta_{1}+\delta_{2}+\cdots+\delta_{l}+\theta_{1}+\theta_{2}+\cdots+$ $\theta_{m} \equiv 0(\bmod n)$. Hence we have $P_{n}^{-}(\widetilde{g}, \tilde{l}, \tilde{m}, \tilde{l}, \tilde{m}) \neq \varnothing$. Then the number 
of elements of $\mathscr{P}_{n}^{-}(\widetilde{g}, \tilde{l}, \tilde{m}, \tilde{l}, \tilde{m})$ is equal to $\{\varphi(d) / 2\} \times C(n ; \chi, m)$.

(II-a) Suppose that $n / 2$ is even and $n / d$ is odd. Then, there is a bijection of $\mathscr{P}_{n}^{-}(\widetilde{g}, \tilde{l}, \tilde{m}, \tilde{l}, \widetilde{m})$ onto $\mathscr{Z}_{n}^{-}(2 ; l, m, \chi, m)_{2}^{0}$. For any element $\left(\delta_{1}, \delta_{2}, \cdots, \delta_{l}, \theta_{1}, \theta_{2}, \cdots, \theta_{m}\right)$ of $D^{-}(n ; \ell, m)$, there are exactly one even nonnegative integer in the set $\{\gamma=i d ; 0 \leqq i \leqq n / d\}$ which satifies $2 \gamma+\delta_{1}+$ $\delta_{2}+\cdots+\delta_{l}+\theta_{1}+\theta_{2}+\cdots+\theta_{m} \equiv 0(\bmod n)$, and exactly one even positive integer in the set $\{\gamma=i d+d / 2 ; 0 \leqq i<n / d\}$ which satisfies $2 \gamma+\delta_{1}+\delta_{2}+\cdots+$ $\delta_{l}+\theta_{1}+\theta_{2}+\cdots+\theta_{m} \equiv 0(\bmod n)$. Then the number of elements of $\mathscr{P}_{n}^{-}(\tilde{g}$, $\widetilde{l}, \tilde{m}, \tilde{l}, \tilde{m})$ is equal to $\{(\varphi(d)+\varphi(d / 2)) / 2\} \times C(n ; \gamma, m)$.

(II-b) Suppose that $n / 2$ is even and $d / 2$ is odd. If $l_{n / 2}=m_{n / 2}=0$, there is a bijection of $\mathscr{P}_{n}^{-}(\tilde{g}, \tilde{l}, \tilde{m}, \tilde{z}, \tilde{m})$ onto $\mathscr{\varkappa}_{n}^{-}(2 ; l, m, \mathcal{\ell}, m)_{2}^{0}$. If $l_{n / 2}+$ $m_{n / 2} \neq 0$, there is a bijection of $\mathscr{P}_{n}^{-}(\widetilde{g}, \tilde{l}, \tilde{m}, \tilde{l}, \tilde{m})$ onto $\mathscr{Z}_{n}^{-}(2 ; l, m, \chi, m)_{2}^{*}$. For any element $\left(\delta_{1}, \delta_{2}, \cdots, \delta_{l}, \theta_{1}, \theta_{2}, \cdots, \theta_{m}\right)$ of $D^{-}(n ; \chi, m)^{0}$ (resp. $D^{-}(n$; $\left.\chi, m)^{*}\right)$ such that $(1 / d)\left(\delta_{1}+\delta_{2}+\cdots+\delta_{l}+\theta_{1}+\theta_{2}+\cdots+\theta_{m}\right)$ is even, there are exactly two (resp. one) even non-negative integers in the set $\{\gamma=i d ; 0 \leqq$ $i \leqq n / d\}$ which satisfy $2 \gamma+\delta_{1}+\delta_{2}+\cdots+\delta_{l}+\theta_{1}+\theta_{2}+\cdots+\theta_{m} \equiv 0 \quad(\bmod n)$. For any element $\left(\delta_{1}, \delta_{2}, \cdots, \delta_{l}, \theta_{1}, \theta_{2}, \cdots, \theta_{m}\right)$ of $D^{-}(n ; \ell, m)^{0} \cup D^{-}(n ; \chi, m)^{*}$ such that $(1 / d)\left(\delta_{1}+\delta_{2}+\cdots+\delta_{l}+\theta_{1}+\theta_{2}+\cdots+\theta_{m}\right)$ is odd, there are no even non-negative integers $\gamma$ satisfying that $2 \gamma+\delta_{1}+\delta_{2}+\cdots+\delta_{l}+\theta_{1}+\theta_{2}+$ $\cdots+\theta_{m} \equiv 0(\bmod n)$. We note $(1 / d)\left(\delta_{1}+\delta_{2}+\cdots+\delta_{l}+\theta_{1}+\theta_{2}+\cdots+\theta_{m}\right)$ is even iff $(1 / 2)\left(\delta_{1}+\delta_{2}+\cdots+\delta_{l}+\theta_{1}+\theta_{2}+\cdots+\theta_{m}\right)$ is even. We have $P_{n}^{-}(\widetilde{g}, \widetilde{l}$, $\tilde{m}, \tilde{\chi}, \tilde{m}) \neq \varnothing$ iff $n, \varnothing$ and $m$ satisfy the condition $(6)_{e}$ in Lemma 6.1. Then the number of elements of $\mathscr{P}_{n}^{-}\left(\widetilde{g}, \tilde{l}, \tilde{m}, \tilde{l}, \tilde{\mathfrak{m}_{k}}\right)$ is equal to

$$
\left\{\begin{array}{lll}
2 \times\{\varphi(d) / 2\} \times C(n ; \chi, m) & \text { if } & l_{n / 2}=m_{n / 2}=0, \\
\{\varphi(d) / 2\} \times C(n ; \chi, m) & \text { if } & l_{n / 2}+m_{n / 2} \neq 0
\end{array}\right.
$$

(II-c) Suppose that $n / 2$ is even, $n / d$ is even and $d / 2$ is even. If $l_{n / 2}=m_{n / 2}=0$, there is a bijection of $\mathscr{P}_{n}^{-}(\widetilde{g}, \widetilde{l}, \tilde{m}, \tilde{q}, \tilde{m})$ onto $\mathscr{Z}_{n}^{-}(2 ; l, m, \mathcal{\chi}$, $\left.\boldsymbol{m}_{2}\right)_{2}^{0}$. If $l_{n / 2}+m_{n / 2} \neq 0$, there is a bijection of $\mathscr{P}_{n}^{-}(\widetilde{g}, \tilde{l}, \tilde{m}, \tilde{z}, \tilde{m})$ onto $\mathscr{Z}_{n}^{-}(2 ; l, m, \chi, m)_{2}^{*}$. For any element $\left(\delta_{1}, \delta_{2}, \cdots, \delta_{l}, \theta_{1}, \theta_{2}, \cdots, \theta_{m}\right)$ of $D^{-}(n$; $\chi, m)^{0}$ (resp. $\left.D^{-}(n ; \chi, m)^{*}\right)$ such that $(1 / d)\left(\delta_{1}+\delta_{2}+\cdots+\delta_{l}+\theta_{1}+\theta_{2}+\cdots+\theta_{m}\right)$ is even, there are exactly two (resp. one) even positive integers in the set $\{\gamma=i d ; 1 \leqq i \leqq n / d\}$ which satisfy $2 \gamma+\delta_{1}+\delta_{2}+\cdots+\delta_{l}+\theta_{1}+\theta_{2}+\cdots+\theta_{m} \equiv 0$ $(\bmod n)$. For any element $\left(\delta_{1}, \delta_{2}, \cdots, \delta_{l}, \theta_{1}, \theta_{2}, \cdots, \theta_{m}\right)$ of $D^{-}(n ; \chi, m)^{0}$ (resp. $\left.D^{-}(n ; \chi, m)^{*}\right)$ such that $(1 / d)\left(\delta_{1}+\delta_{2}+\cdots+\delta_{l}+\theta_{1}+\theta_{2}+\cdots+\theta_{m}\right)$ is odd, there are exactly two (resp. one) even positive integers in the set $\{\gamma=$ $i d+d / 2 ; 0 \leqq i<n / d\}$ which satisfy $2 \gamma+\delta_{1}+\delta_{2}+\cdots+\delta_{l}+\theta_{1}+\theta_{2}+\cdots+\theta_{m} \equiv 0$ $(\bmod n)$. Hence we have $P_{n}^{-}(\widetilde{g}, \tilde{l}, \tilde{m}, \tilde{l}, \tilde{m}) \neq \varnothing$. Then the number of elements of $\mathscr{P}_{n}^{-}\left(\widetilde{g}, \tilde{l}, \tilde{m}, \tilde{z}, \widetilde{m_{R}}\right)$ is equal to; 


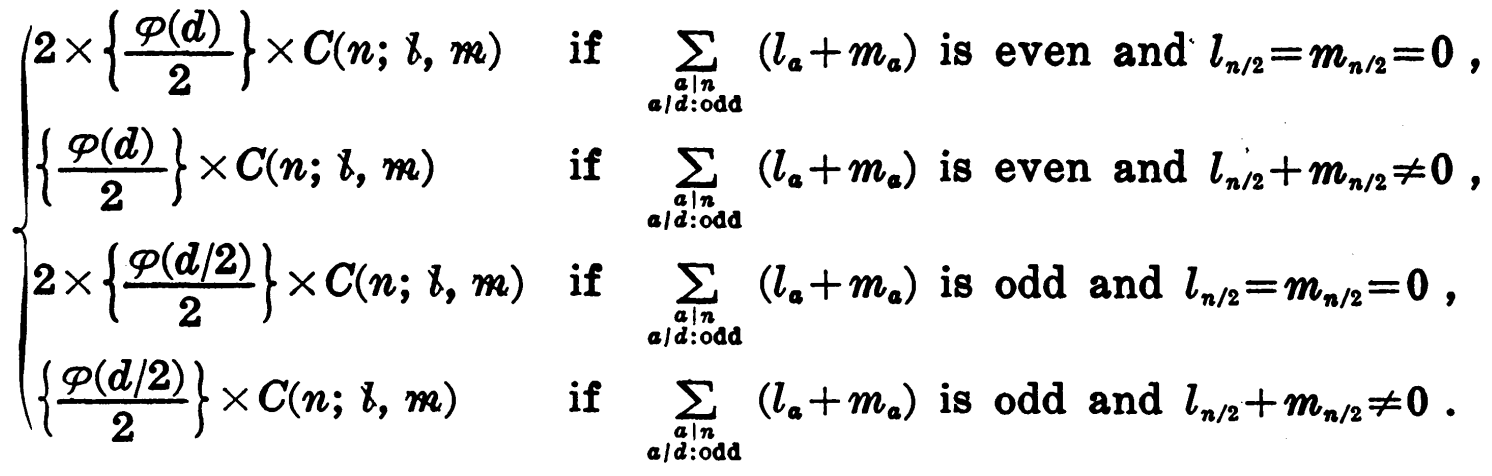

Correction to "Classification of Periodic Maps on Compact Surfaces I' (Tokyo J. Math., 6 (1983), 75-94).

We will correct the formula of $Q_{0}(n ; l, m)$ in page 87 as follows;

$$
\left\{\begin{array}{c}
\left(\begin{array}{c}
{\left[\frac{n}{2}\right]+\frac{m}{2}-1} \\
\frac{m}{2}
\end{array}\right)\left(\begin{array}{c}
{\left[\frac{n}{2}\right]+\left[\frac{l}{2}\right]} \\
{\left[\frac{l}{2}\right]}
\end{array}\right) \quad \text { if } m \text { is even ; } \\
\left(\begin{array}{c}
\frac{n}{2}+\frac{m-1}{2}-1 \\
\frac{m-1}{2}
\end{array}\right)\left(\begin{array}{c}
\frac{n}{2}+\left[\frac{l-1}{2}\right] \\
{\left[\frac{l-1}{2}\right]}
\end{array}\right) \text { if } n \text { is even, } m \text { is odd and } l \geqq 1 ; \\
\text { otherwise ; }
\end{array}\right.
$$

Other miscellaneous errata are as follows:

p. 78, $1 \downarrow 7:$ (2) g.c.d. $\left\{\alpha_{1}, \beta_{1}, \alpha_{2}, \beta_{2}, \cdots, \alpha_{g}, \beta_{g}, \delta_{1}, \delta_{2}, \cdots, \delta_{l}, \theta_{1}, \theta_{2}, \cdots\right.$, $\left.\theta_{m}\right\} \equiv 1(\bmod n)$ should be read (2) g.c.d. $\left\{\alpha_{1}, \beta_{1}, \alpha_{2}, \beta_{2}, \cdots, \alpha_{g}, \beta_{g}, \delta_{1}, \delta_{2}, \cdots\right.$, $\left.\delta_{l}, \theta_{1}, \theta_{2}, \cdots, \theta_{m}, n\right\}=1$.

p. 82, $1 \uparrow 4: \quad \sigma_{j}=h \psi h^{-1}$ should be read $\sigma_{j}=h \varphi h^{-1}$.

p. 86, $1 \downarrow 7$ : g.c.d. $\left\{\delta_{1}, \delta_{2}, \cdots, \delta_{l}, \theta_{1}, \theta_{2}, \cdots, \theta_{m}\right\} \equiv 1 \quad(\bmod n)$ should be read g.c.d. $\left\{\delta_{1}, \delta_{2}, \cdots, \delta_{l}, \theta_{1}, \theta_{2}, \cdots, \theta_{m}, n\right\}=1$.

p. 89, 1 $\uparrow 2: \quad g(X)=\{2 g-2-(n-1)+2 n\} / 2 n$ should be read $g(X)=$ $\left\{2 g-2-(n-1)\left(l_{1}+m\right)+2 n\right\} / 2 n$.

[3] in References should be read;

[3] S. Suzuki, On homeomorphisms of a 3-dimensional handlebody, Canad. J. Math., 29, (1977), 111-124.

\section{References}

[1] T. Asor, Classification of free involutions on surfaces, Hiroshima Math. J., 6 (1976), 171-181. 
[2] D. R.J. Chillingworth, A finite set of generators for the homeotopy group of a nonorientable surfaces, Proc. Cambridge Philos. Soc., 65 (1969), 409-430.

[ 3 ] W. R. B. LICKORISH, Homeomorphisms of non-orientable two-manifolds, Proc. Cambridge Philos. Soc., 59 (1963), 307-317.

[4] P. A. SMitH, Abelian actions on 2-manifolds, Michigan Math. J., 14 (1967), 257-275.

[5] Shin'ichi SUzUKI, On homeomorphisms of a 3-dimensional handlebody, Canad. J. Math., 29 (1977), 111-124.

[6!] G. T. WhybuRn, Analytic topology, Amer. Math. Soc. Colloquium Publ., 28, Amer. Math. Soc., Providence, 1942.

[7] K. Yoxoyama, Classification of periodic maps on compact surfaces I, Tokyo J. Math., 6 (1983), 75-94.

[8] K. Yoxoyama, Complete classification of periodic maps on compact surfaces, preprint.

Present Adress:

DEPARTMENT OF Mathematics

SOPHIA UNIVERSITY

KiOI-CHO, ChIYODA-RU, TORYo 102 